\title{
Error estimate for the upwind scheme for the linear transport equation with boundary data
}

\author{
Nina Aguillon, Franck Boyer ${ }^{\dagger}$
}

December 15, 2016

\begin{abstract}
We study the upwind finite volume scheme on a general mesh for the initial and boundary-value problem associated with a linear transport equation. For any BV initial and boundary data we prove the (optimal) convergence rate $1 / 2$ in the $L^{1}$-norm. Compared to previous works, our main contribution is to take into account the boundary data and to relax some regularity assumptions on the velocity field.

As an intermediate result, we also provide a complete proof of the BV regularity of weak solutions of such a general transport problem.
\end{abstract}

\section{Introduction}

\subsection{Framework and main result}

This paper is a contribution to the analysis of the upwind finite volume scheme for the approximation of weak solutions of the following linear transport problem

$$
\left\{\begin{array}{lll}
\partial_{t} u(t, x)+\mathbf{v}(t, x) \cdot \nabla_{x} u(t, x)=0, & t \in(0, T), & x \in \Omega, \\
u(0, x)=u^{0}(x), & x \in \Omega, \\
u(t, x)=u_{b}(t, x), & t \in(0, T), \quad x \in \Gamma, \text { where } \mathbf{v}(t, x) \cdot \mathbf{n}(x)<0 .
\end{array}\right.
$$

The final time $T>0$, the velocity field $\mathbf{v}$, the initial data $u^{0}$ and the (inflow) boundary data $u_{b}$ are given. The domain $\Omega \subset \mathbb{R}^{d}$ is assumed to be bounded and at least of class $\mathcal{C}^{3}$; its boundary is denoted by $\Gamma=\partial \Omega$ and $\mathbf{n}$ is the outward unit normal. We shall use the following notations

$$
\Omega^{T}:=(0, T) \times \Omega, \quad \Gamma^{T}:=(0, T) \times \Gamma .
$$

We present in details the upwind finite volume scheme for this problem in Section 3 . At that stage, we only point out that the convergence analysis for this problem has already been done in [Boy12 under general assumptions, whereas an error estimate was obtained for instance in [MV07] in the case where the problem is posed in the whole space $\Omega=\mathbb{R}^{d}$ (that is without boundary data) and for a Lipschitz continuous advection field v. In this work, we would like to prove the same kind of error estimates as in MV07 but for the full problem (1) taking into account the boundary data. Moreover, our assumptions on the velocity field are weaker than the one in [MV07] since we allow time discontinuities for $\mathbf{v}$.

Let us remark, in this introduction, that the presence of a boundary data really makes the structure of the solution $u$ more complicated so that the error analysis is not a straightforward extension of previous works, even though our proof is strongly inspired by the one in [MV07.

*Laboratoire Jacques-Louis Lions, UMR 7598, Université Pierre et Marie Curie,75252 Paris Cedex 05, France, aguillon@ljll.math.upmc.fr

i'Institut de Mathématiques de Toulouse, UMR 5219, Université de Toulouse, CNRS, UPS IMT, F-31062 Toulouse Cedex 9, France, franck.boyer@math.univ-toulouse.fr 
- First, even if $u^{0}$ and $u_{b}$ are smooth and compatible at time $t=0$, this regularity is not inherited at all times.

As an example let us consider $\Omega=(0,+\infty) \subset \mathbb{R}, u_{b}=0$ and $u^{0} \in \mathcal{C}^{\infty}(\Omega)$ compactly supported in $\Omega$. Let $a>0$ such that $u^{0}(a) \neq 0$. We take $\mathbf{v}$ that depends only on time as follows $\mathbf{v}(t)=2(t-a) / a$.

For $0<t<a$, we have $\mathbf{v}(t)<0$, and thus there is no inflow boundary. The exact solution is thus given by

$$
u(t, x)=u^{0}\left(x-\int_{0}^{t} \mathbf{v}(s) d s\right)=u^{0}\left(x+2 t-t^{2} / a\right),
$$

and in particular, at time $t=a$, we have $u(a, 0)=u^{0}(a) \neq 0$. At time $t=a$, the velocity field $\mathbf{v}$ changes it sign and thus the inflow boundary data has to be taken into account. The solution becomes

$$
u(t, x)=u^{0}\left(x+2 t-t^{2} / a\right) \mathbf{1}_{\left\{x>2 t-t^{2} / a-a\right\}},
$$

and it is clear that it is no more continuous in space for any time $t>a$. The solution is not smooth anymore and thus, even if one regularize the data by any convolution process, we cannot expect that the associated exact solution of (1) for those regularized data will be smooth. Observe that the regularity of $\mathbf{v}$ is not important in this behavior but only the fact that the inflow/outflow parts of the boundary may depend on time.

Actually, the suitable assumption we shall need in our analysis is the fact that the inflow part of the boundary is of finite perimeter (see $10 p)$.

- Observe that, even though the solution clearly does not depend on the values of $u_{b}$ outside the inflow part of the boundary, we will require in assumption (3) some regularity of $u_{b}$ on the whole boundary $\Gamma^{T}$. If one is only given the values of $u_{b}$ on the inflow part of the boundary, this amounts to require that there exists some extension of this data on the whole boundary $\Gamma^{T}$ that satisfies (3).

It seems that this assumption is necessary to guarantee that the solution $u$ of the transport problem has a bounded total variation as illustrated in Remark 2.7 given in the sequel.

- Provided that (3) and suitable assumptions on $\mathbf{v}$ (weaker than the ones we need for the error analysis) hold, we can prove that the solution $u$ of (1) has a bounded total variation. This BV regularity result for the initial- and boundary-value transport problem does not seem to be available in the literature in the form we need. This property, that has some interest by itself, is thus proved in an independent Section 7

Let us now state the regularity assumptions we need on the data to perform the error analysis:

$$
\begin{gathered}
u^{0} \in \mathrm{BV}(\Omega) \cap L^{\infty}(\Omega), \\
u_{b} \in \mathrm{BV}\left(\Gamma^{T}\right) \cap L^{\infty}\left(\Gamma^{T}\right), \\
\left\{\begin{array}{l}
\operatorname{div} \mathbf{v}=0, \text { in } \Omega^{T}, \\
\mathbf{v} \in \operatorname{BV}\left(\Omega^{T}\right) \cap L^{\infty}\left(\Omega^{T}\right), \\
L_{\mathbf{v}}:=\left\|\nabla_{x} \mathbf{v}\right\|_{L^{\infty}\left(\Omega^{T}\right)}<+\infty .
\end{array}\right.
\end{gathered}
$$

The last assumption says that $\mathbf{v}$ is Lipschitz-continuous in space, uniformly in time, but we allow time discontinuities for $\mathbf{v}$.

Roughly speaking, if $u_{\Delta x}$ is the approximate solution given by the upwind finite volume scheme, our main result will be the following.

Theorem. Assume that the data and the mesh satisfy suitable regularity assumptions and that the time step fulfills a strong Courant-Friedrichs-Lewy condition, then we have the estimate

$$
\left\|u-u_{\Delta x}\right\|_{L^{\infty}\left(0, T ; L^{1}(\Omega)\right)}+\iint_{\Gamma^{T}}\left|u-u_{\Delta x} \| \mathbf{v} \cdot \mathbf{n}\right| d \sigma d t \leq C \sqrt{\Delta x} .
$$

The precise statement is given in Theorem 4.1, where the hypothesis are described and discussed and the trace terms are suitably defined. 


\subsection{State of the art}

The estimation of the order of convergence for the linear transport equation on a general mesh has a long history, going back to Kuznetsov in the 70's. Let us briefly describe some of the existing bibliography. We restrict our attention to results on the linear transport equation or involving a boundary data, discretized with a finite volume method. A wider bibliography extended to nonlinear conservation laws or other numerical methods can be found in those papers.

- Advection equation with Lipschitz continuous velocity field on $\mathbb{R}^{d}$. When the initial data (and hence the exact solution) is smooth and when the mesh is Cartesian, the Lax-Wendroff theorem yields the convergence at order 1 of the scheme. On non-Cartesian grids, the Lax-Wendroff theorem does not apply because first, the scheme is not consistent in the finite difference sense and second, no uniform BV bound holds in general (see the counterexample of Després [Des04a]).

However a $1 / 2$ convergence rate can be proved in various norms and for different regularity assumptions on the data. This was proved in the pioneering work [Kuz76] on Cartesian meshes, in [VV03 for Friedrichs systems, in [Des04b, Des04a] for constant velocity fields, and in MV07, Mer08 for space and time Lipschitz continuous velocity fields. Using a probabilistic interpretation of the scheme, similar results are obtained in DL11 for time independent but non divergence free velocity fields.

Let us underline that, on unstructured grids, the order $1 / 2$ is optimal even though the initial data is smooth, see the example of Peterson Pet91.

In some cases, the first order convergence can be recovered with additional hypothesis on the mesh, see [BGP05. We will not tackle this problem here and will focus on the case of general meshes and BV data.

- Advection equation with Lipschitz continuous velocity field on a bounded domain. To our knowledge, this work is the first to give the optimal order $1 / 2$ on a bounded domain with general data. The influence of the boundary is studied in CVV00 for Friedrichs system, but the solution is assumed to be smooth. In [CH99] and [Vig96 orders of convergence are proved in the framework of scalar conservation laws; the nonlinearity of the flux introduces many difficulties and the best convergence rate obtained so far is $1 / 4$. Moreover, the fact that the exact solution has bounded total variation is quite hard to obtain. In the case of a homogeneous boundary condition (i.e. $u_{b}=0$ ), this is proved in BlRN79, but the extension to non-homogeneous Dirichlet condition is quite difficult, as underlined in CR15, and requires regularity on $u_{b}$ and some compatibility conditions, see [CR15, Theorem 2.7]. In the case of the linear transport equation we are able to give a simpler and more general proof, see Section 7 of the present paper.

- Less regular velocity field. When the velocity field $\mathbf{v}$ is not Lipschitz continuous, the exact solution cannot be obtained by the method of characteristics and it is necessary to build a new framework in which (1) is well-posed. Within the theory of DiPerna-Lions, the convergence of the scheme (with no evaluation of the order of convergence) is proved in Boy12 whereas a 1/2 order of convergence for a suitable distance that metrizes the weak topology is proved in [SS16]. When $\mathbf{v}$ is one-sided Lipschitz continuous, an optimal order of convergence in the Wasserstein distance has recently been obtained in [DLV16] on Cartesian meshes. Those cases are beyond the scope of this paper.

In this historical landscape, our work is the closest to MV07 since we extend their result to the case where a boundary data has to be taken into account. We actually follow the sketch of their proof. However, due to the influence of the boundary that complicates the structure of the solution, this extension is not straightforward. In particular we propose a new way to regularize the exact solution that is adapted to the treatment of boundary terms and which, additionally, requires less regularity on the vector field $\mathbf{v}$. We will enlighten throughout the paper the key differences and novelties compared to this reference.

The paper is organized as follows. We continue this introduction with some notation and some useful results on BV functions. In Section 2 we expose some results on the exact solution of (1). Some of them are well known (weak formulation, renormalization properties) and other are new (Lipschitz continuity of the solution in the normal direction near the boundary, see Proposition 2.4). The upwind scheme and the assumptions 
on the mesh are presented in Section 3. Our main result, Theorem 4.1, is stated in Section 4 together with the intermediate results that we need during its proof. The results concerning the regularization by a space convolution of the exact solution are gathered in Section 5 . Section 6 is devoted to some estimates related to the properties of the discretization method. The curved boundary and the low regularity in time of the considered functions complicate the analysis. Eventually, in Section 7 (that can be read somehow independently) we propose a proof of the BV regularity property stated in Theorem 2.5 and that we use all along the paper.

\subsection{Notation and useful results}

Averages and measures In the sequel, if $(X, \mu)$ is any measurable set with $\mu(X)<+\infty$ and if $\Phi$ belongs to $L^{1}(X, \mu)$, we denote by

the average of $\Phi$ over $X$.

$$
f_{X} \Phi d \mu:=\frac{1}{\mu(X)} \int_{X} \Phi d \mu
$$

We shall use the notation $\mathcal{H}_{d-1}$ for the $(d-1)$-dimensional Hausdorff measure.

BV functions The natural regularity class we shall deal with in this paper is the one of bounded variation functions. Let us recall the main definitions and properties that we need and that one can find, for instance, in $\mathrm{AFP00}$.

A function $f \in L^{1}(U)$ defined on an open set $U$ of $\mathbb{R}^{N}$ with Lipschitz continuous boundary is said to be of bounded variation if and only if

$$
\operatorname{TV}_{U}(f):=\sup \left\{\int_{U} f \operatorname{div} \Phi d x, \text { for } \Phi \in \mathcal{C}_{c}^{\infty}\left(U, \mathbb{R}^{N}\right),\|\Phi\|_{L^{\infty}(U)} \leq 1\right\}<+\infty .
$$

The space of such functions is denoted by $\mathrm{BV}(U)$ and is equipped with the following norm

$$
\|f\|_{\mathrm{BV}(U)}:=\|f\|_{L^{1}(U)}+\mathrm{TV}_{U}(f) .
$$

Observe that the total variation is semi-continuous in the following sense : for any $f \in L^{1}(U)$ and $\left(f_{n}\right)_{n} \subset L^{1}(U)$ we have

$$
\left\|f_{n}-f\right\|_{L^{1}(U)} \underset{n \rightarrow \infty}{\longrightarrow} 0 \Longrightarrow \mathrm{TV}_{U}(f) \leq \liminf _{n \rightarrow \infty} \mathrm{TV}_{U}\left(f_{n}\right)
$$

We note that $W^{1,1}(U) \subset \mathrm{BV}(U)$ and that for such functions, we have $\operatorname{TV}_{U}(f)=\|\nabla f\|_{L^{1}(U)}$. Moreover, the set $\mathcal{C}^{\infty}(\bar{U})$ is weakly dense in $\mathrm{BV}(U)$ in the following sense

$$
\begin{aligned}
& \text { For any } f \in \mathrm{BV}(U) \text {, there exists a sequence }\left(f_{n}\right)_{n} \in \mathcal{C}^{\infty}(\bar{U}) \text { such that } \\
& \left\|f_{n}-f\right\|_{L^{1}(U)} \underset{n \rightarrow \infty}{\longrightarrow} 0, \text { and } \operatorname{TV}_{U}\left(f_{n}\right)=\left\|\nabla f_{n}\right\|_{L^{1}(U)} \underset{n \rightarrow \infty}{\longrightarrow} \operatorname{TV}_{U}(f) .
\end{aligned}
$$

Moreover, if $f \in \operatorname{BV}(U) \cap L^{\infty}(U)$, then the sequence $\left(f_{n}\right)_{n}$ can be chosen to satisfy $\left\|f_{n}\right\|_{L^{\infty}(U)} \leq 2\|f\|_{L^{\infty}(U)}$.

The trace operator $\gamma_{\partial U}: f \in \mathcal{C}^{\infty}(\bar{U}) \mapsto f_{\mid \partial U} \in L^{1}(U)$ can be continuously extended to $\mathrm{BV}(U)$ in the following sense : for any $f \in \mathrm{BV}(U)$ and $\left(f_{n}\right)_{n} \subset \mathrm{BV}(U)$, we have

$$
\left\|f_{n}-f\right\|_{L^{1}(U)}+\left|\mathrm{TV}_{U}\left(f_{n}\right)-\mathrm{TV}_{U}(f)\right| \underset{n \rightarrow \infty}{\longrightarrow} 0 \Longrightarrow\left\|\gamma_{\partial U} f_{n}-\gamma_{\partial U} f\right\|_{L^{1}(\partial U)} \underset{n \rightarrow \infty}{\longrightarrow} 0 .
$$

One can naturally extend all the previous definitions and properties to define the space BV on smooth manifolds such as $\Gamma^{T}=(0, T) \times \Gamma$.

We shall need at some point the following property that can be easily obtained first for smooth functions and then extended by a density argument.

Lemma 1.1. For any $R>0, h>0$, we set $K=(-R, R)^{d-1} \times[0, h), L=(-R, R)^{d-1} \times(-h, 0]$, and $\sigma=(-R, R)^{d-1} \times\{0\}$. We have

$$
(2 R)^{d-1}\left|f_{K} f d x-f_{L} f d x\right| \leq \mathrm{TV}_{K \cup L}(f), \quad \forall f \in \mathrm{BV}(K \cup L),
$$

and

$$
(2 R)^{d-1}\left|f_{K} f d x-f_{\sigma} \gamma_{\sigma} f d \sigma\right| \leq \mathrm{TV}_{K}(f), \quad \forall f \in \mathrm{BV}(K) .
$$


Level sets of BV functions, sets of finite perimeter We say that a subset $E$ of $\Omega$ has a finite perimeter if

$$
\operatorname{Per}_{\Omega}(E):=\operatorname{TV}_{\Omega}\left(\mathbf{1}_{E}\right)<+\infty .
$$

The main property we will use is that the level sets of a BV function are of finite perimeter and we can relate the function to its level sets through the following construction: for any $f \in \mathrm{BV}(\Omega)$ and any $\eta \in \mathbb{R}$ we define

$$
\chi_{f}^{\eta}= \begin{cases}\mathbf{1}_{\{f>\eta\}}, & \text { if } \eta>0, \\ -\mathbf{1}_{\{f<\eta\}}, & \text { if } \eta<0, \\ 0, & \text { if } \eta=0,\end{cases}
$$

and the following result holds.

Theorem 1.2. With the previous notation, for any $f \in \mathrm{BV}(\Omega)$ we have:

1. If $f \in L^{\infty}(\Omega)$, then $\chi_{f}^{\eta}=0$ for any $\eta$ such that $|\eta|>\|f\|_{L^{\infty}(\Omega)}$.

2. The Fubini theorem gives that

$$
f=\int_{\mathbb{R}} \chi_{f}^{\eta} d \eta \quad \text { in } L^{1}(\Omega), \quad \text { and } \quad\|f\|_{L^{1}(\Omega)}=\int_{\mathbb{R}}\left\|\chi_{f}^{\eta}\right\|_{L^{1}(\Omega)} d \eta .
$$

3. Co-aera formula : the total variation of $f$ can be expressed as follows

$$
\operatorname{TV}_{\Omega}(f)=\int_{\mathbb{R}} \operatorname{TV}_{\Omega}\left(\chi_{f}^{\eta}\right) d \eta=\int_{\mathbb{R}} \operatorname{Per}_{\Omega}(\{f>\eta\}) d \eta
$$

We shall use similar notation and results when $\Omega$ is replaced by $\Omega^{T}$ or by the manifold $\Gamma^{T}$.

Normal and tangential coordinates Since $\Omega$ is assumed to be a bounded $\mathcal{C}^{3}$ domain of $\mathbb{R}^{d}$, we can use the following notations and results (see for instance Amb00, Boy05, BF13]).

For any $\xi>0$, we introduce the sets (see Figure 1)

$$
\mathcal{O}_{\xi}:=\{x \in \Omega, d(x, \Gamma)<\xi\}, \text { and } \Gamma_{\xi}:=\{x \in \Omega, d(x, \Gamma)=\xi\}
$$

There exists a $\xi_{\Omega}>0$ such that, on $\overline{\mathcal{O}_{2 \xi_{\Omega}}}$, the distance map $d(., \Gamma)$ is smooth, and the orthogonal projection $P_{\Gamma}$ onto $\Gamma$ is well-defined and smooth. We recall that $P_{\Gamma}(x)$ is the unique point in $\Gamma$ such that $d(x, \Gamma)=d\left(x, P_{\Gamma} x\right)$.

We consider a $\mathcal{C}^{\infty}$ map $\eta: \bar{\Omega} \rightarrow \mathbb{R}$ such that $0 \leq \eta \leq 1, \eta=1$ on $\mathcal{O}_{\xi_{\Omega}}$ and $\eta=0$ in $\Omega \backslash \mathcal{O}_{2 \xi_{\Omega}}$ and we set

$$
\mathbf{n}(x)=-\eta(x)(\nabla d(x, \Gamma)), \quad \forall x \in \Omega .
$$

It follows that $\mathbf{n}$ is a smooth vector field on $\bar{\Omega}$ such that $\|\mathbf{n}\|_{L^{\infty}\left(\Omega^{T}\right)} \leq 1$ and which satisfies

$$
\mathbf{n}(x)=\mathbf{n}\left(P_{\Gamma}(x)\right), \quad \text { and } \quad x=P_{\Gamma}(x)-d(x, \Gamma) \mathbf{n}\left(P_{\Gamma}(x)\right), \quad \forall x \in \mathcal{O}_{\xi_{\Omega}},
$$

so that, the following map is a diffeomorphism

$$
(s, \tilde{x}) \in\left[0, \xi_{\Omega}\right] \times \Gamma \mapsto \tilde{x}-s \mathbf{n}(\tilde{x}) \in \overline{\mathcal{O}_{\xi_{\Omega}}} .
$$

With those notations we can use normal and tangential coordinates to make computations near the boundary of $\Omega$. More precisely, there exists a smooth function $J:\left[0, \xi_{\Omega}\right] \times \Gamma \rightarrow \mathbb{R}$, depending on the geometry of the manifold $\Gamma$, such that $J(0, \tilde{x})=1$ for any $\tilde{x} \in \Gamma, \inf _{\left[0, \xi_{\Omega}\right] \times \Gamma} J>0$ and such that the following change of variable formula holds

$$
\left\{\begin{aligned}
\int_{\Gamma_{\xi}} f(x) d \sigma(x) & =\int_{\Gamma} f(\tilde{x}-\xi \mathbf{n}(\tilde{x})) J(\xi, \tilde{x}) d \sigma(\tilde{x}), \quad \forall \xi \in\left[0, \xi_{\Omega}\right] \\
\int_{\mathcal{O}_{\xi}} f(x) d x & =\int_{0}^{\xi} \int_{\Gamma} f(\tilde{x}-s \mathbf{n}(\tilde{x})) J(s, \tilde{x}) d \sigma(\tilde{x}) d s, \quad \forall \xi \in\left[0, \xi_{\Omega}\right] .
\end{aligned}\right.
$$




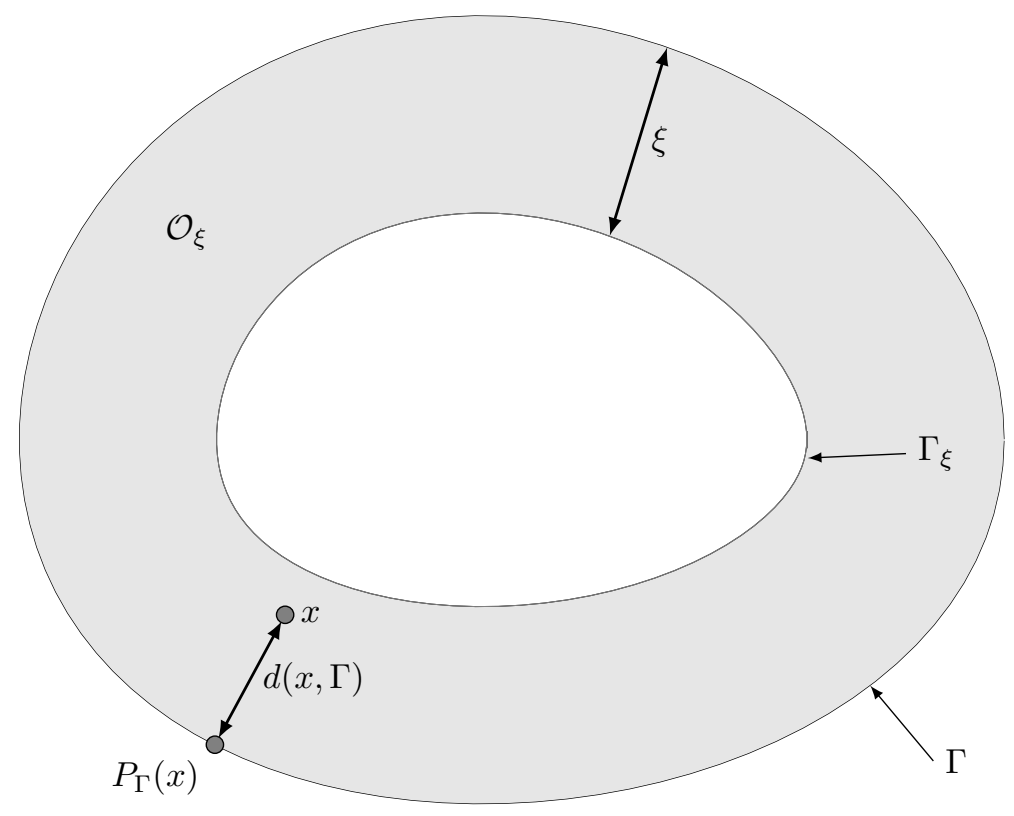

Figure 1: Notations near the boundary of $\Omega$

\section{Weak solutions of the transport problem}

We gather here some results and notation concerning weak solutions of the initial and boundary problem for the transport equation (1).

In this work, we assume that the velocity field $\mathbf{v}$ is bounded and Lipschitz continuous in space, see (4). Therefore, we may try to solve (1) by the method of characteristics that we recall here. We define by $X$ the flow associated with $\mathbf{v}$ (or, more precisely, any smooth extension of $\mathbf{v}$ on the whole space) as the solution of the following Cauchy problem

$$
\left\{\begin{array}{l}
\partial_{t} X\left(t ; t_{0}, x_{0}\right)=\mathbf{v}\left(t, X\left(t ; t_{0}, x_{0}\right)\right) \\
X\left(t_{0} ; t_{0}, x_{0}\right)=x_{0}
\end{array}\right.
$$

A simple computation shows that any (smooth enough) solution of (1) is constant along any portion of the characteristics curves that is included in $\bar{\Omega}$. If $\mathbf{v}$ is tangent to the boundary, or more generally if $\mathbf{v} \cdot \mathbf{n} \geq 0$ (here the divergence-free condition is not necessary) all the backward characteristics are included in $\Omega$ and thus we obtain the exact formula

$$
u(t, x)=u^{0}(X(0 ; t, x)), \quad \forall t \in(0, T), \forall x \in \Omega .
$$

In the case we are interested in, that is when the velocity field $\mathbf{v}$ enters the domain on some part of the boundary, we need to account for the boundary data and we obtain

$$
u(t, x)= \begin{cases}u_{b}\left(t^{0}(t, x), X\left(t^{0}(t, x) ; t, x\right)\right) & \text { if } t^{0}(t, x)>0 \\ u^{0}(X(0 ; t, x)) & \text { otherwise }\end{cases}
$$

where

$$
t^{0}(t, x)=\inf \{s \in[0, t], \text { such that } X(\tau ; t, x) \in \Omega, \forall \tau \in[s, t]\},
$$

is the time at which the backward characteristic leaves the space time domain $(0, T) \times \Omega$.

As in the introduction, we clearly see again in Formula (9) that even if $u^{0}$ and $u_{b}$ are smooth, the solution $u$ may not be smooth. This is one of the reasons why we cannot reduce the analysis to smooth solutions and need to cope with weak solutions of the transport problem. Observe that we will not use the formula (9) at any point in the paper, in particular because the entering time $t^{0}$ is difficult to handle. 
The advection field $\mathbf{v}$ being given, we can define the inflow and outflow parts of the boundary as follows

$$
\Gamma_{\text {in }}^{T}:=\left\{(t, x) \in \Gamma^{T}, \mathbf{v}(t, x) \cdot \mathbf{n}(x)<0\right\}, \text { and } \Gamma_{\text {out }}^{T}:=\left\{(t, x) \in \Gamma^{T}, \mathbf{v}(t, x) \cdot \mathbf{n}(x)>0\right\} .
$$

For our error analysis to hold, we shall need the following assumption

$$
\Gamma_{\text {in }}^{T} \text { is of finite perimeter in } \Gamma^{T} \text {. }
$$

We can now state the following existence and uniqueness result for weak solutions of Problem (1). This result is valid under more general assumptions than the one we adopted in the introduction and its proof can be found in Boy05, BF13.

Theorem 2.1. Assume that $\Omega$ is a bounded Lipschitz domain and $\mathbf{v} \in L^{1}\left(0, T ;\left(W^{1,1}(\Omega)\right)^{d}\right)$ such that div $\mathbf{v}=0$.

For any $u^{0} \in L^{\infty}(\Omega), u_{b} \in L^{\infty}\left(\Gamma^{T}\right)$ there exists a unique function $u \in L^{\infty}\left(\Omega^{T}\right) \cap \mathcal{C}^{0}\left([0, T], L^{1}(\Omega)\right)$ and a function $\gamma u \in L^{\infty}\left(\Gamma^{T}\right)$, referred to as the trace of $u$, which is unique almost everywhere on $\Gamma_{\text {in }}^{T} \cup \Gamma_{\text {out }}^{T}$ and such that

$$
u(0, .)=u^{0}, \quad \text { and } \gamma u=u_{b}, \text { almost everywhere in } \Gamma_{\mathrm{in}}^{T},
$$

and

$$
\iint_{\Omega^{T}} u\left(\partial_{t} \varphi+\mathbf{v} \cdot \nabla_{x} \varphi\right) d x d t+\int_{\Omega} u^{0} \varphi(0, .) d x-\int_{\Omega} u(T, .) \varphi(T, .) d x-\iint_{\Gamma^{T}}(\gamma u) \varphi \mathbf{v} \cdot \mathbf{n} d \sigma d t=0
$$

for any $\varphi \in W^{1,1}([0, T] \times \bar{\Omega})$.

Note that we can always change $u_{b}$ on $\Gamma^{T} \backslash \Gamma_{\text {in }}^{T}$, by truncation, in such a way that $\left\|u_{b}\right\|_{L^{\infty}\left(\Gamma^{T}\right)} \leq\|u\|_{L^{\infty}\left(\Omega^{T}\right)}$.

In practice, we will often use the following formulation in which the inflow and outflow parts of the boundary are explicitly separated

$$
\begin{aligned}
\iint_{\Omega^{T}} u\left(\partial_{t} \varphi+\mathbf{v} \cdot \nabla_{x} \varphi\right) d x d t+\int_{\Omega} u^{0} \varphi(0, .) d x-\int_{\Omega} u(T, .) \varphi(T, .) d x \\
\quad-\iint_{\Gamma_{\text {in }}^{T}} u_{b} \varphi \mathbf{v} \cdot \mathbf{n} d \sigma d t-\iint_{\Gamma_{\text {out }}^{T}}(\gamma u) \varphi \mathbf{v} \cdot \mathbf{n} d \sigma d t=0 .
\end{aligned}
$$

In addition to the previous well-posedness result, we can also state the following renormalization properties for the weak solutions that will be useful.

Theorem 2.2. Under the same assumptions as in the previous theorem and for any piecewise Lipschitz continuous function $\beta: \mathbb{R} \rightarrow \mathbb{R}$ we have that $\beta(u)$ is the unique weak solution of (1) associated with the data $\beta\left(u^{0}\right)$ and $\beta\left(u_{b}\right)$. Moreover, we have the trace equality $\gamma(\beta(u))=\beta(\gamma(u))$ almost everywhere on $\Gamma_{\text {in }}^{T} \cup \Gamma_{\text {out }}^{T}$.

As a consequence of those properties, we deduce the following result.

Corollary 2.3. Assume the same hypothesis as in Theorem 2.1.

- Let $E \subset \mathbb{R}$ be a set such that $u^{0} \in E$, almost everywhere in $\Omega$, and $u_{b} \in E$ almost everywhere in $\Gamma_{\text {in }}^{T}$. Then, we have $u \in E$ (resp. $\gamma u \in E$ ) almost everywhere in $\Omega^{T}$ (resp. almost everywhere in $\Gamma_{\text {out }}^{T}$ ).

In particular the maximum principle holds: $u$ and $\gamma u$ take their values in the interval $\left[u_{\min }, u_{\max }\right]$, with

$$
u_{\min }:=\min \left(\underset{\Omega}{\operatorname{essinf}} u^{0}, \underset{\Gamma^{T}}{\operatorname{essinf}} u_{b}\right), \quad \text { and } u_{\max }:=\max \left(\underset{\Omega}{\operatorname{ass} \sup } u^{0}, \underset{\Gamma^{T}}{\operatorname{ess} \sup } u_{b}\right) .
$$

- The following $L^{2}$-energy balance equation holds

$$
\int_{\Omega}|u(T, .)|^{2} d x+\iint_{\Gamma_{\text {out }}^{T}}|\gamma u|^{2}|\mathbf{v} \cdot \mathbf{n}| d \sigma d t=\int_{\Omega}\left|u^{0}\right|^{2} d x+\iint_{\Gamma_{\text {in }}^{T}}\left|u_{b}\right|^{2}|\mathbf{v} \cdot \mathbf{n}| d \sigma d t .
$$


We also refer to Boy05, BF13 for the proofs of such results. Notice that the same result is true if one uses the infimum and the supremum of $u_{b}$ only on $\Gamma_{\text {in }}^{T}$ in 12 but since this is no more the case for the approximate solution (see Proposition 3.3) , we choose to use here this definition of $u_{\min }$ and $u_{\max }$.

Finally, to obtain the error estimate, we will use some regularity properties for the exact solution of our transport problem with initial and boundary data. The first needed result concerns the continuity of the solution near the boundary with respect to the normal coordinate. Such a continuity result was proved under general assumptions in Boy05, but we shall need here a sharper estimate of the Lipschitz kind that we can state as follows.

Proposition 2.4. Assume that (2), (3) and (4) hold as well as the finite perimeter condition (10). Then there exists $C>0$, depending only on $\Omega, T$ and $\mathbf{v}$, such that the unique weak solution $u$ of the transport equation (1) satisfies, for any $0<\xi<\xi_{\Omega}$, the estimate

$$
\iint_{\Gamma_{\mathrm{in}}^{T}}\left|u_{b}(t, \tilde{x})-u(t, \tilde{x}-\xi \mathbf{n}(\tilde{x}))\right||\mathbf{v}(t, \tilde{x}) \cdot \mathbf{n}(\tilde{x})| d \sigma(\tilde{x}) d t \leq C\left(\operatorname{Per}_{\Gamma^{T}}\left(\Gamma_{\mathrm{in}}^{T}\right)+\operatorname{TV}_{\Gamma^{T}}\left(u_{b}\right)+\|u\|_{L^{\infty}\left(\Omega^{T}\right)}\right) \xi .
$$

Actually, we shall prove a slightly more general result in Section 5.2

The second result we need is the $L^{\infty}(0, T ; \mathrm{BV}(\Omega))$ regularity of the solution.

Theorem 2.5. Let $\Omega$ be a bounded $\mathcal{C}^{3}$ domain of $\mathbb{R}^{d}$. Suppose that $u^{0}$ satisfies (2), $u_{b}$ satisfies (3) and $\mathbf{v}$ satisfies (4), then the unique weak solution $u$ of the transport problem (1) belongs to $L^{\infty}(0, T ; \mathrm{BV}(\Omega))$. More precisely, we have $u(t,.) \in \mathrm{BV}(\Omega)$ for any $t \in[0, T]$ and

$$
\sup _{t \in[0, T]} \operatorname{TV}_{\Omega}(u(t, .)) \leq C\left(\left\|\gamma_{\Gamma} u^{0}-u_{b}(0, .)\right\|_{L^{1}(\Gamma)}+\operatorname{TV}_{\Omega}\left(u^{0}\right)+\operatorname{TV}_{\Gamma^{T}}\left(u_{b}\right)\right)
$$

where $C$ depends only on $\Omega, \mathbf{v}$ and $T$.

Remark 2.6. It can be easily deduced from this result that the solution $u$ actually belongs to $\mathrm{BV}((0, T) \times \Omega)$.

Actually, we shall often use the following weaker version of the estimate

$$
\sup _{t \in[0, T]} \operatorname{TV}_{\Omega}(u(t, .)) \leq C\left(\|u\|_{L^{\infty}\left(\Omega^{T}\right)}+\operatorname{TV}_{\Omega}\left(u^{0}\right)+\operatorname{TV}_{\Gamma^{T}}\left(u_{b}\right)\right)
$$

A complete proof of this result does not seem to be available in the literature, that is the reason why we give such a proof in Section 7. We cannot directly use the result of [BlRN79] since it requires much higher regularity on the boundary data $u_{b}$, see also CR15. Our strategy of proof is to first deal with the case where $\Omega$ is a half-space: here we introduce a uniform Cartesian discretization of $\Omega$ and prove a uniform discrete BV estimate on the approximate solution, then we pass to the limit, in the spirit of [CH99] on the whole space and OV06] for polyhedral domains. Finally we recover the result on the general domain $\Omega$ by using suitable change of variables.

We will actually prove a slightly more precise result where neither the BV regularity of $\mathbf{v}$ nor the divergencefree condition are required.

Remark 2.7. The fact that $u_{b}$ is BV on the whole boundary $\Gamma^{T}$ and not only on the inflow part $\Gamma_{\text {in }}^{T}$ is actually a necessary condition for this theorem to hold, as illustrated by the following example.

Consider the domain $\Omega=(0,+\infty)$. For any $k \geq 1$ we set $\tau_{k}=\frac{2}{3} 2^{-k}, T_{1}=0$, and for $k \geq 2, T_{k}=$ $\sum_{j=1}^{k-1} \frac{3}{2} \tau_{j}=1-2^{-(k-1)}$. Then, we choose any $v_{k}>0$ such that $\sum_{k>1} v_{k}<+\infty$.

We define the space independent velocity field $\mathbf{v}(t, x):(0,1) \times \Omega \rightarrow \mathbb{R}$ as follows

$$
\mathbf{v}(t, x)= \begin{cases}v_{k} & \text { for } t \in\left(T_{k}, T_{k}+\tau_{k}\right) \\ -v_{k} & \text { for } t \in\left(T_{k}+\tau_{k}, T_{k}+\frac{3}{2} \tau_{k}\right)\end{cases}
$$

The velocity field is thus oriented in the inflow direction during a time interval $\tau_{k}$ then in the outflow direction during a time interval $\tau_{k} / 2$. Since $\mathbf{v}$ does not depend on the space variable $x$, and $\sum_{k \geq 1} v_{k}<+\infty$, it is clear that $\mathbf{v}$ satisfies (4) (at least away from infinity) and we have

$$
\Gamma_{\text {in }}^{T}=\bigcup_{k \geq 1}\left(T_{k}, T_{k}+\tau_{k}\right) \times\{0\}
$$




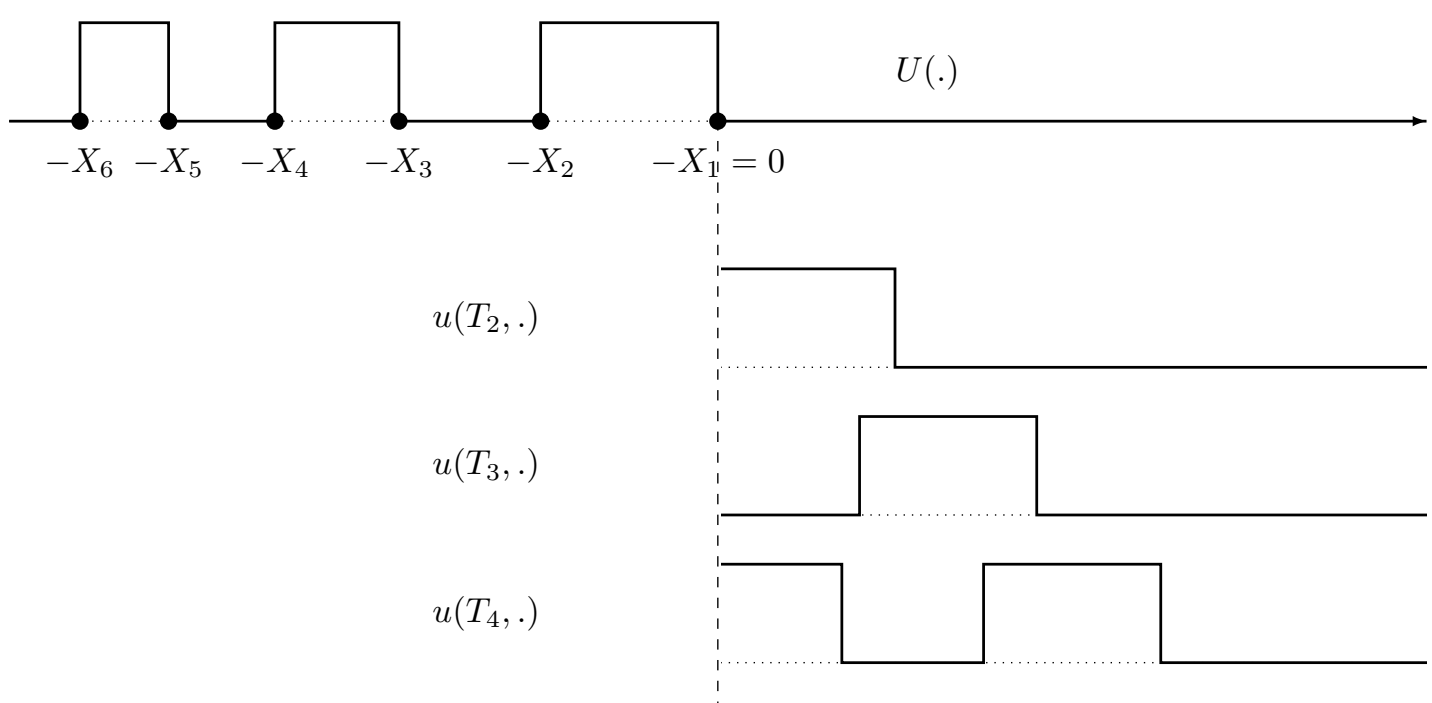

Figure 2: Description of the solution exhibited in Remark 2.7

Consider now the initial data $u^{0}=0$ and the boundary data $u_{b}$ defined on $\Gamma_{\text {in }}^{T}$ by

$$
u_{b}(t)=\left(1+(-1)^{k-1}\right) / 2, \text { for } t \in\left(T_{k}, T_{k}+\tau_{k}\right),
$$

which means that $u_{b}(t)=1$ on $\left(T_{k}, T_{k}+\tau_{k}\right)$ if $k$ is odd, and $u_{b}(t)=0$ otherwise. Observe that $u_{b} \in \operatorname{BV}\left(\Gamma_{\text {in }}^{T}\right)$ but there is no function $\overline{u_{b}} \in \mathrm{BV}\left(\Gamma^{T}\right)$ such that $\overline{u_{b}}=u_{b}$ on $\Gamma_{\text {in }}^{T}$.

The exact solution of the transport problem for those data can be computed explicitly, see Figure 2, To this end we set $X_{1}=0$ and

$$
X_{k}=\sum_{j=1}^{k-1} \frac{1}{2} v_{j} \tau_{j}, \quad \forall k \geq 2,
$$

and we define for any $x \in \mathbb{R}$,

$$
U(x)= \begin{cases}0 & \text { for } x>0 \\ 1 & \text { for }-X_{k+1}<x<-X_{k} \text { with } k \geq 1 \text { odd } \\ 0 & \text { for }-X_{k+1}<x<-X_{k} \text { with } k \geq 2 \text { even. }\end{cases}
$$

Then, the exact solution $u$ satisfies, for any $k$ and any $x>0$,

$$
u\left(T^{k}, x\right)=U\left(x-X_{k}\right) .
$$

As a consequence, the total variation of $u\left(T^{k},.\right)$ is simply given by

$$
\operatorname{TV}_{\Omega}\left(u\left(T^{k}, .\right)\right)=k-1 \text {. }
$$

Since $T_{k} \rightarrow 1$ as $k \rightarrow \infty$, we deduce that

$$
\sup _{t<1} \operatorname{TV}_{\Omega}(u(t, .))=+\infty .
$$

This counterexample is in fact valid on a (large enough) bounded domain: indeed the sum $\sum_{j=1}^{+\infty} \frac{1}{2} v_{j} \tau_{j}$ is finite and thus the sequence $\left(X_{k}\right)_{k}$ is bounded, which proves that the support of the solution $u$ is bounded.

\section{The upwind scheme}

Let us now introduce the numerical method that we want to analyze in this paper. It is one of the most classical and simple schemes to approximate the solution of transport problems, the explicit-in-time upwind scheme. 


\subsection{The finite volume mesh}

We discretize the domain $\Omega$ with a mesh $\mathcal{T}$ made of disjoint cells (the notation for a generic cell is $K$ ) and such that $\bar{\Omega}=\cup_{K \in \mathcal{T}} \bar{K}$. The interior cells, namely the ones such that $\mathcal{H}_{d-1}(\Gamma \cap \partial K)=0$, are supposed to be polyhedral, and convex (however this assumption is not essential and could be somehow relaxed), the set of such cells is referred to as $\mathcal{T}^{\text {int }}$. However, since the domain $\Omega$ is not assumed to be polyhedral, we do not make such assumptions for boundary cells that may possess curved faces and may not be convex. The set of such boundary cells is referred to as $\mathcal{T}^{\text {ext }}=\mathcal{T} \backslash \mathcal{T}^{\text {int }}$.

For any $K \in \mathcal{T},|K|$ will be its Lebesgue measure, and we shall also denote by $\partial K$ the set of its faces. A generic face in the mesh will be referred to as $\sigma$, and we set $|\sigma|=\mathcal{H}_{d-1}(\sigma)$ its area. The set of all the faces in the mesh in denoted by $\partial \mathcal{T}$; the set of the faces included in $\Gamma$ is denoted by $\partial \mathcal{T}^{\text {ext }}$ and finally $\partial \mathcal{T}^{\text {int }}=\partial \mathcal{T} \backslash \partial \mathcal{T}$ ext is the set of the interior faces. The notations are illustrated on Figure 3.

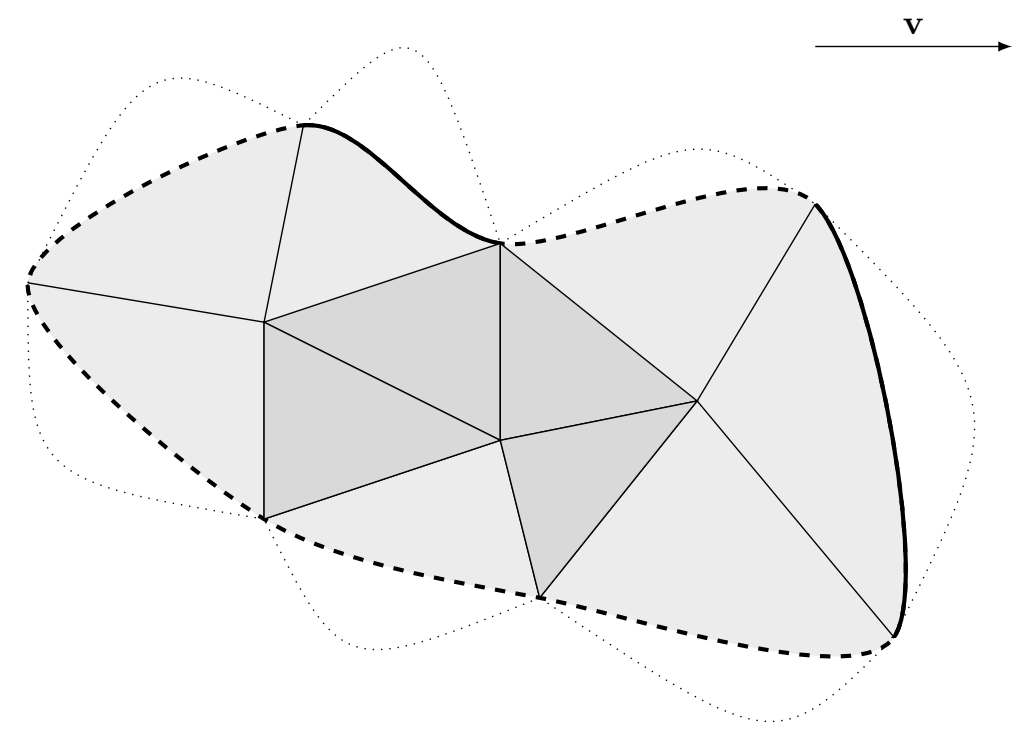

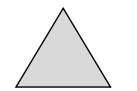

interior cells in $\mathcal{T}^{\text {int }}$

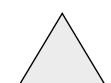

exterior cells in $\mathcal{T}^{\text {ext }}$

virtual cells in $\mathcal{T}^{*}$

outflow edges in $\partial \mathcal{T}_{+}^{n}$

- . - . inflow edges in $\partial \mathcal{T}_{-}^{n}$

interior edges in $\partial \mathcal{T}^{\text {int }}$

Figure 3: Notations attached to a mesh and to an example of velocity field $\mathbf{v}$

The mesh size $\Delta x$ is the maximum of the diameters of the cells in the mesh. Since $\Delta x$ is meant to tend to 0 , we shall additionally assume that

$$
\Delta x \leq \xi_{\Omega}^{2}
$$

We assume the following somehow standard regularity properties of the mesh : there exists some $\alpha>0$ such that

$$
\begin{gathered}
K \text { contains a ball of radius } \alpha \operatorname{diam}(K), \quad \forall K \in \mathcal{T}, \\
\operatorname{Card}(\partial K) \leq \frac{1}{\alpha}, \quad \forall K \in \mathcal{T} \\
\sum_{\sigma \in \partial K}|\sigma| \operatorname{diam}(K) \leq \frac{1}{\alpha}|K|, \quad \forall K \in \mathcal{T} .
\end{gathered}
$$

Actually, since the interior cells are supposed to be convex one can prove that, for the interior mesh (17) is a consequence of assumptions $(15)$ and $(16)$. However, for exterior cells, (17) needs to be added to the set of assumptions. Most of the estimates in this paper will depend on the regularity parameter $\alpha$.

We shall need the following lemma which is proved in [DE06, Lemma 6.3] and uses the fact that interior cells are supposed to be convex. 
Lemma 3.1. There exists a $C>0$ depending only ond and $\alpha$ (appearing in (15)) such that for any $f \in H^{1}(\Omega)$ we have

$$
\left(f_{K} f d x-f_{\sigma} f d \sigma\right)^{2} \leq \frac{C \operatorname{diam}(K)}{|\sigma|} \int_{K}|\nabla f|^{2} d x, \quad \forall K \in \mathcal{T}^{\text {int }}, \forall \sigma \in \partial K .
$$

The one-dimensional version of this result will also be useful and reads

$$
\left|\frac{1}{|b-a|} \int_{a}^{b} f(t) d t-f(a)\right|^{2} \leq|b-a| \int_{a}^{b}\left|f^{\prime}(t)\right|^{2} d t, \quad \forall f \in H^{1}(a, b), \quad \forall a<b .
$$

We shall finally use the following result which can be proved exactly as in [Dro03, Lemma 7.1].

Lemma 3.2. There exists a $C>0$ depending only on $d$ such that for any convex non empty open set $U \subset \mathbb{R}^{d}$ we have

$$
\int_{U}\left|f(x)-\left(f_{U} f d y\right)\right| d x \leq C \frac{(\operatorname{diam}(U))^{d+1}}{|U|} \int_{U}|\nabla f| d x, \quad \forall f \in W^{1,1}(U) .
$$

If two cells $K$ and $L$ share a face, we denote this face as $K \mid L$, so that $K \mid L \in \partial K$. To each exterior face $\sigma \in \partial \mathcal{T}^{\text {ext }}$ we associate a virtual cell $L \subset \bar{\Omega}^{c}$ such that we can write $\sigma=K \mid L$. We denote by $\mathcal{T}^{*}$ the set of all the virtual cells we just introduced, the extended mesh being $\mathcal{T} \cup \mathcal{T}^{*}$. Those virtual cells are only a notation whose interest is to ease the writing of some computations but their precise shape do not enter the analysis at any point.

We denote by $\mathbf{n}_{K L}$ the unit vector normal to $K \mid L$, oriented outward of $K$, this is a constant vector excepted on the exterior faces that may be non-flat.

The constant time step is chosen as $\Delta t=T / N$, for some $N \in \mathbb{N}$ and it will be classically constrained by a CFL stability condition, see (23) below. Additionally, in order to measure the error only with respect to the mesh size, we shall assume that

$$
\Delta t \leq \frac{1}{\alpha} \operatorname{diam}(K), \quad \forall K \in \mathcal{T} .
$$

where $\alpha$ is the same parameter as in 15 - (17), even if it means to take a smaller value of this parameter.

For $n \in \mathbb{N}$ we set $t^{n}=n \Delta t$, and we denote by $K^{n}$ the time-space cell $K^{n}=\left(t^{n}, t^{n+1}\right) \times K$, and similarly for the time-space faces $K^{n}\left|L^{n}=\left(t^{n}, t^{n+1}\right) \times K\right| L, \sigma^{n}=\left(t^{n}, t^{n+1}\right) \times \sigma$.

\subsection{Approximation of the data}

We discretize the initial and the boundary data by setting

$$
u_{K}^{0}:=f_{K} u^{0} d x, \forall K \in \mathcal{T}, \quad \text { and } \quad u_{b, \sigma}^{n}:=\oiint_{\sigma^{n}} u_{b}(t, x) d \sigma(x) d t, \forall n<N, \forall \sigma \in \partial \mathcal{T}^{\text {ext }},
$$

and

$$
u_{\Delta x}^{0}:=\sum_{K \in \mathcal{T}} u_{K}^{0} \mathbf{1}_{K} \in L^{\infty}(\Omega), \quad u_{b, \Delta x}:=\sum_{n<N} \sum_{\sigma \in \partial \mathcal{T} \text { ext }} u_{b, \sigma}^{n} \mathbf{1}_{\sigma^{n}} \in L^{\infty}\left(\Gamma^{T}\right) .
$$

We introduce the average velocity fluxes across time-space faces by setting

It is easily seen that

$$
V_{K L}^{n}:=\oiint_{K^{n} \mid L^{n}} \mathbf{v}(t, x) \cdot \mathbf{n}_{K L} d \sigma(x) d t, \quad \forall n \in \mathbb{N}, \forall K \mid L \in \partial \mathcal{T} .
$$

$$
V_{K L}^{n}=-V_{L K}^{n} \text {, for any } n \in \mathbb{N} \text { and any face } K \mid L \in \partial \mathcal{T} \text {. }
$$

With this definition, we can introduce, for any $n \in \mathbb{N}, K \in \mathcal{T}$, the sets

$$
K_{-}^{n}:=\left\{L \in \mathcal{T} \cup \mathcal{T}^{*} \text {, s.t. } K \mid L \in \partial \mathcal{T}, V_{K L}^{n}<0\right\} \quad \text { and } \quad K_{+}^{n}:=\left\{L \in \mathcal{T} \cup \mathcal{T}^{*} \text {, s.t. } K \mid L \in \partial \mathcal{T}, V_{K L}^{n} \geq 0\right\}
$$

of the inflow (resp. outflow) neighboring cells of $K$ during the time interval $\left(t^{n}, t^{n+1}\right)$, including the virtual cells for the boundary cells.

We will also need at some point to distinguish the inflow (resp. outflow) exterior faces so that we introduce the following notations

$$
\partial \mathcal{T}_{-}^{n}:=\left\{K \mid L \in \partial \mathcal{T}^{\mathrm{ext}}, V_{K L}^{n}<0\right\}, \text { and } \partial \mathcal{T}_{+}^{n}:=\left\{K \mid L \in \partial \mathcal{T}^{\mathrm{ext}}, V_{K L}^{n} \geq 0\right\}
$$




\subsection{The upwind scheme}

With the above notations, the explicit upwind scheme classically writes

$$
u_{K}^{n+1}-u_{K}^{n}+\frac{\Delta t}{|K|} \sum_{L \in K_{-}^{n}}|K| L\left|V_{K L}^{n} u_{L}^{n}+\frac{\Delta t}{|K|} \sum_{L \in K_{+}^{n}}\right| K|L| V_{K L}^{n} u_{K}^{n}=0 .
$$

By the Stokes formula, the fact that $\operatorname{div} \mathbf{v}=0$ yields the conservativity property $\sum_{L \in \partial K}|K| L \mid V_{K L}^{n}=0$, or equivalently

$$
\sum_{L \in K_{-}^{n}}|K| L\left|V_{K L}^{n}=-\sum_{L \in K_{+}^{n}}\right| K|L| V_{K L}^{n}
$$

so that we can write the scheme as follows:

$$
u_{K}^{n+1}-u_{K}^{n}+\frac{\Delta t}{|K|} \sum_{L \in K_{-}^{n}}|K| L \mid V_{K L}^{n}\left(u_{L}^{n}-u_{K}^{n}\right)=0 .
$$

It remains to clarify what $u_{L}^{n}$ is if $L \in \mathcal{T}^{*}$ is a virtual cell. Since the boundary condition in (1) is only imposed on the inflow part of the boundary, we naturally proceed as follows: let $K \in \mathcal{T}$ be the unique boundary cell in the mesh $\mathcal{T}$ such that $K \mid L \in \partial \mathcal{T}^{\text {ext }}$, then the value chosen for $u_{L}^{n}$ depends on whether the flow is, in average, entering or exiting the domain through this face:

$$
u_{L}^{n}= \begin{cases}u_{b, K \mid L}^{n} & \text { if } L \in K_{-}^{n} \\ u_{K}^{n} & \text { if } L \in K_{+}^{n}\end{cases}
$$

We recall below the classical $L^{\infty}$-stability result for the upwind scheme. Its proof is easily seen from Formula 21.

Proposition 3.3. Suppose that for some $\lambda$ in $(0,1)$, the following strict Courant-Friedrichs-Lewy condition holds

$$
\forall n \geq 0, \forall K \in \mathcal{T}, \quad \Delta t \sum_{L \in K_{-}^{n}}|K| L|| V_{K L}^{n}|\leq(1-\lambda)| K \mid .
$$

Then the numerical solution verifies the discrete maximum principle

$$
u_{\min } \leq u_{K}^{n} \leq u_{\max }, \quad \forall n \geq 0, \forall K \in \mathcal{T}
$$

where $u_{\min }$ and $u_{\max }$ are defined in 12 .

Notice that the stability result still holds for $\lambda=0$ but we will need this stronger assumption in order to take benefit of a small but positive amount of numerical dissipation of the scheme. The same assumption was made in MV07.

Let us denote by $u_{\Delta x}\left(\right.$ resp $\left.\gamma u_{\Delta x}\right)$ the piecewise constant function on $\Omega^{T}$ (resp. on $\Gamma^{T}$ ) associated to the scheme (21)- 22 .

$$
\begin{gathered}
u_{\Delta x}:=\sum_{n<N} \sum_{K \in \mathcal{T}} u_{K}^{n} \mathbf{1}_{K^{n}} \in L^{\infty}\left(\Omega^{T}\right), \\
\gamma u_{\Delta x}:=\sum_{n<N} \sum_{K \mid L \in \mathcal{T}_{-}^{n}} u_{b, K \mid L}^{n} \mathbf{1}_{K^{n} \mid L^{n}}+\sum_{n<N} \sum_{K \mid L \in \mathcal{T}_{+}^{n}} u_{K}^{n} \mathbf{1}_{K^{n} \mid L^{n}} \in L^{\infty}\left(\Gamma^{T}\right) .
\end{gathered}
$$

Observe that $\gamma u_{\Delta x}$ plays the role of the trace of $u_{\Delta x}$ and that the equality $\gamma u_{\Delta x}=u_{b, \Delta x}$ only holds on the inflow boundary faces as expected.

In the proof of the error estimate we shall use discrete $L^{2}$-energy estimates that we recall below.

Definition 3.4. We define the total discrete $L^{2}$-energy balance of the scheme on the whole time interval as the quantity

$$
\mathcal{E}_{\Delta x}\left(u_{\Delta x}, T\right):=\int_{\Omega}\left(u_{\Delta x}^{0}\right)^{2} d x-\int_{\Omega}\left(u_{\Delta x}(T, .)\right)^{2} d x-\iint_{\Gamma^{T}}\left(\gamma u_{\Delta x}\right)^{2}(\mathbf{v} \cdot \mathbf{n}) d \sigma d t
$$


and the numerical $L^{2}$-dissipation of the scheme as the quantity

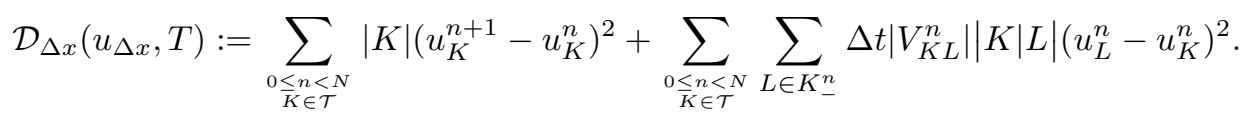

Theorem 3.5. The solution of the upwind scheme satisfies the following energy equality

$$
\mathcal{E}_{\Delta x}\left(u_{\Delta x}, T\right)=-\sum_{\substack{0 \leq n<N \\ K \in \mathcal{T}}}|K|\left(u_{K}^{n+1}-u_{K}^{n}\right)^{2}+\sum_{\substack{0 \leq n<N \\ K \in \mathcal{T}}} \sum_{L \in K_{-}^{n}} \Delta t\left|V_{K L}^{n}\right||K| L \mid\left(u_{L}^{n}-u_{K}^{n}\right)^{2} .
$$

Moreover, assuming the strict CFL condition (23), we have the estimate

$$
\mathcal{D}_{\Delta x}\left(u_{\Delta x}, T\right) \leq \frac{2}{\lambda} \mathcal{E}_{\Delta x}\left(u_{\Delta x}, T\right) .
$$

It proves in particular that $\mathcal{E}_{\Delta x}\left(u_{\Delta x}, T\right) \geq 0$, i.e. the $L^{2}$-stability of the scheme.

Proof. By definition of $u_{\Delta x}, \gamma u_{\Delta x}$ and $V_{K L}^{n}$, we easily check that

$$
\begin{aligned}
\mathcal{E}_{\Delta x}\left(u_{\Delta x}, T\right)=\sum_{K \in \mathcal{T}}|K|\left(u_{K}^{0}\right)^{2} & -\sum_{K \in \mathcal{T}}|K|\left(u_{K}^{N}\right)^{2} \\
& -\sum_{0 \leq n<N} \sum_{K \mid L \in \partial \mathcal{T}_{-}^{n}} \Delta t\left(u_{L}^{n}\right)^{2}|K| L\left|V_{K L}^{n}-\sum_{0 \leq n<N} \sum_{K \mid L \in \partial \mathcal{T}_{+}^{n}} \Delta t\left(u_{K}^{n}\right)^{2}\right| K|L| V_{K L}^{n} .
\end{aligned}
$$

The proof of 26 is very classical. It is obtained by multiplying the scheme 21$]$ by $u_{K}^{n}$, summing the result over $n$ and $K$ and finally by using conservativity properties of the numerical fluxes to reorganize the contribution of each face. This is exactly the same formula (but with slightly different notations) than the one given in Boy12, Formula (3.13)]. Notice however that there is a typo in the above reference where a factor $\Delta t$ should be removed in front of the time diffusion term (the one containing $u_{K}^{n+1}-u_{K}^{n}$ ).

Inequality (27) is proved in [MV07] but without the boundary terms. We thus give a new proof here which, in addition, is shorter than the one in the above reference.

Let us define the two non-negative terms

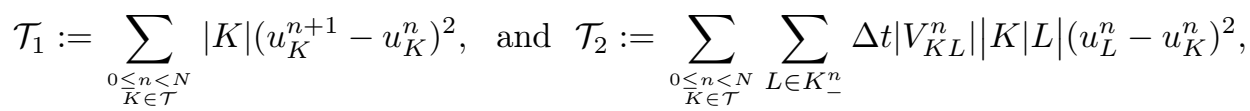

so that, by definition, we have

$$
\mathcal{E}_{\Delta x}\left(u_{\Delta x}, T\right)=-\mathcal{T}_{1}+\mathcal{T}_{2} \text {, and } \mathcal{D}_{\Delta x}\left(u_{\Delta x}, T\right)=\mathcal{T}_{1}+\mathcal{T}_{2}
$$

We just have to prove that $\mathcal{T}_{1}$ can be suitably controlled by $\mathcal{T}_{2}$. To this end, for a given $K$ and $n$, we take the square of the formula (21) defining the scheme to get

$$
\left(u_{K}^{n+1}-u_{K}^{n}\right)^{2}=\frac{\Delta t^{2}}{|K|^{2}}\left(\sum_{L \in K_{-}^{n}}|K| L \mid V_{K L}^{n}\left(u_{L}^{n}-u_{K}^{n}\right)\right)^{2},
$$

that we write in the following way

$$
\left(u_{K}^{n+1}-u_{K}^{n}\right)^{2}=\frac{\Delta t^{2}\left(\sum_{L \in K_{-}^{n}}|K| L \mid V_{K L}^{n}\right)^{2}}{|K|^{2}}\left(\frac{\sum_{L \in K_{-}^{n}}|K| L \mid V_{K L}^{n}\left(u_{L}^{n}-u_{K}^{n}\right)}{\sum_{L \in K_{-}^{n}}|K| L \mid V_{K L}^{n}}\right)^{2} .
$$

By definition, we have $V_{K L}^{n}<0$ for any $L \in K_{-}^{n}$ and we can thus use the Jensen inequality in the last factor to get

$$
\left(u_{K}^{n+1}-u_{K}^{n}\right)^{2} \leq \frac{\Delta t^{2}\left(\sum_{L \in K_{-}^{n}}|K| L|| V_{K L}^{n} \mid\right)}{|K|^{2}}\left(\sum_{L \in K_{-}^{n}}|K| L|| V_{K L}^{n} \mid\left(u_{L}^{n}-u_{K}^{n}\right)^{2}\right),
$$


and then, by using the CFL condition (23), we obtain

$$
\left(u_{K}^{n+1}-u_{K}^{n}\right)^{2} \leq(1-\lambda) \frac{\Delta t}{|K|}\left(\sum_{L \in K_{-}^{n}}|K| L|| V_{K L}^{n} \mid\left(u_{L}^{n}-u_{K}^{n}\right)^{2}\right) .
$$

Observe that we implicitly assumed that $\sum_{L \in K_{-}^{n}}|K| L|| V_{K L}^{n} \mid>0$ in this computation, but in the case where this sum vanishes we easily see that all the terms $V_{K L}^{n}$ are zero and thus $u_{K}^{n+1}-u_{K}^{n}=0$ and finally (28) still holds.

Multiplying (28) by $|K|$ and summing over $n$ and $K$ leads to the inequality

$$
\mathcal{T}_{1} \leq(1-\lambda) \mathcal{T}_{2}
$$

We can then conclude by writing

$$
\mathcal{E}_{\Delta x}\left(u_{\Delta x}, T\right)=-\mathcal{T}_{1}+\mathcal{T}_{2} \geq \lambda \mathcal{T}_{2} \geq \frac{\lambda}{2(1-\lambda)} \mathcal{T}_{1}+\frac{\lambda}{2} \mathcal{T}_{2} \geq \frac{\lambda}{2}\left(\mathcal{T}_{1}+\mathcal{T}_{2}\right)=\frac{\lambda}{2} \mathcal{D}_{\Delta x}\left(u_{\Delta x}, T\right)
$$

\section{Main result and sketch of the proof}

The aim of this paper is to bound the $L^{1}$-error between the exact solution (and its trace) of the transport equation (1) and the approximate solution (and its trace) given by the upwind scheme (21).

We denote by $E\left(u, u_{\Delta x}, T\right)$ the total error at time $T$ defined by

$$
E\left(u, u_{\Delta x}, T\right):=\int_{\Omega}\left|u-u_{\Delta x}\right|(T, .) d x+\iint_{\Gamma^{T}}\left|\gamma u-\gamma u_{\Delta x}\right||\mathbf{v} \cdot \mathbf{n}| d \sigma d s .
$$

The rest of the paper is devoted to the proof of the following error estimate theorem.

Theorem 4.1. Assume that $u^{0}$ satisfies (2), $u_{b}$ satisfies (3) and $\mathbf{v}$ satisfies (4). Moreover, we assume that the inflow boundary satisfies (10). Finally, we assume that the mesh satisfies the regularity assumptions (15)-(17) and that the CFL condition (23) holds for some $\lambda \in(0,1)$. Then there exists $a C>$, depending only on $\Omega$, $T, \mathbf{v}, \alpha$ and $\lambda$, such that

$$
E\left(u, u_{\Delta x}, T\right) \leq C\left(\left(1+\operatorname{Per}_{\Gamma^{T}}\left(\Gamma_{\text {in }}^{T}\right)\right)\|u\|_{L^{\infty}\left(\Omega^{T}\right)}+\operatorname{TV}_{\Omega}\left(u^{0}\right)+\operatorname{TV}_{\Gamma^{T}}\left(u_{b}\right)\right) \sqrt{\Delta x}
$$

Let us comment the different hypothesis of Theorem 4.1 .

- Hypothesis (10) allows us to deal with the parts of the boundary where $\mathbf{v} \cdot \mathbf{n}$ changes its sign. This assumption is particularly useful in the proof of Proposition 2.4. even though we might expect this proposition to hold even without this assumption.

- Concerning the hypothesis on the velocity field $\mathbf{v}$, we underline that they are weaker than in [MV07, in which $\mathbf{v}$ is supposed to be Lipschitz regular both in space and time. Here discontinuities in time are allowed, since we only assume that $\mathbf{v}$ has bounded total variation in time (and space).

In this section we give the proof of Theorem 4.1 and state all the intermediate results we need. Most of them are proved in the next sections. In what follows, the letter $C$ denotes a generic constant that may differ from line to line. In any case, the values of $C$ will not depend on $\Delta t, \Delta x$ nor on the regularization parameter $\epsilon$ that we will introduce later on. 


\subsection{Restriction to indicatrix boundary and initial conditions}

We shall use the BV regularity assumption on the data to reduce the problem to the case where the initial and boundary data are characteristic functions of sets of finite perimeter. We use here the notations of Section 1.3 .

From the renormalization property given in Theorem 2.2 it is easily seen that, for any $\eta \in \mathbb{R}$, the unique weak solution to the transport problem (1) associated with the initial data $\chi_{u^{0}}^{\eta}$ and the boundary data $\chi_{u_{b}}^{\eta}$ is nothing but the function $\chi_{u}^{\eta}$; moreover its trace is $\gamma\left(\chi_{u}^{\eta}\right)=\chi_{\gamma u}^{\eta}$.

Let us now define $u_{\Delta x}^{\eta}$ to be the unique solution of the numerical scheme associated with the initial data $\chi_{u^{0}}^{\eta}$ and the boundary data $\chi_{u_{b}}^{\eta}$. Observe that $u_{\Delta x}^{\eta}$ is not, in general, a characteristic function due to the numerical diffusion of the scheme.

However, since the discretization of the data and the scheme itself are linear, we easily obtain from Theorem 1.2 that the approximate solution $u_{\Delta x}$ can be written as

$$
u_{\Delta x}=\int_{\mathbb{R}} u_{\Delta x}^{\eta} d \eta
$$

It follows that the error $E\left(u, u_{\Delta x}, T\right)$ can be bounded as follows

$$
E\left(u, u_{\Delta x}, T\right) \leq \int_{\mathbb{R}} E\left(\chi_{u}^{\eta}, u_{\Delta x}^{\eta}, T\right) d \eta=\int_{|\eta| \leq\|u\|_{L^{\infty}\left(\Omega^{T}\right)}} E\left(\chi_{\Delta x}^{\eta}, u^{\eta}, T\right) d \eta .
$$

As a consequence of the co-area formula (Theorem 1.2, we see that Theorem 4.1 will be proved if we manage to obtain the following estimate

$$
E\left(\chi_{u}^{\eta}, u_{\Delta x}^{\eta}, T\right) \leq C\left(1+\operatorname{Per}_{\Gamma^{T}}\left(\Gamma_{\text {in }}^{T}\right)+\operatorname{Per}_{\Omega}\left(\left\{u^{0}>\eta\right\}\right)+\operatorname{Per}_{\Gamma^{T}}\left(\left\{u_{b}>\eta\right\}\right)\right) \sqrt{\Delta x},
$$

for some $C$ independent of $\eta$.

Finally, we are led to prove the following result.

Theorem 4.2. Under the same assumptions as in Theorem 4.1, there exists a $C>0$ depending only on $\Omega$, $T, \mathbf{v}, \alpha$ and $\lambda$, such that for any set $E^{0} \subset \Omega$ (resp. $E_{b} \subset \Gamma^{T}$ ) of finite perimeter in $\Omega$ (resp. in $\left.\Gamma^{T}\right)$, we have

$$
E\left(u, u_{\Delta x}, T\right) \leq C\left(1+\operatorname{Per}_{\Gamma^{T}}\left(\Gamma_{\text {in }}^{T}\right)+\operatorname{Per}_{\Omega}\left(E^{0}\right)+\operatorname{Per}_{\Gamma^{T}}\left(E_{b}\right)\right) \sqrt{\Delta x},
$$

where $u$ (resp. $u_{\Delta x}$ ) is the solution of the transport problem (1) (resp. of the upwind scheme (21)) associated with the initial data $u^{0}=\mathbf{1}_{E^{0}}$ and the boundary data $u_{b}=\mathbf{1}_{E_{b}}$.

In this result, $u^{0}$ and $u_{b}$ are characteristic functions of finite perimeter sets. This implies in particular, by Corollary 2.3 that under the CFL condition (23), we have

$$
\left\{\begin{array}{l}
u(t, x) \in\{0,1\}, \text { and } u_{\Delta x}(t, x) \in[0,1], \text { for almost every }(t, x) \in \Omega^{T}, \\
\gamma u(t, x) \in\{0,1\}, \text { and } \gamma u_{\Delta x}(t, x) \in[0,1], \text { for almost every }(t, x) \in \Gamma^{T}
\end{array}\right.
$$

\subsection{Numerical weak formulation. Expression of the $L^{1}$-error}

The approximate solution $u_{\Delta x}$ and its trace $\gamma u_{\Delta x}$ are bounded (thanks to the maximum principle, see Proposition 3.3 but obviously do not satisfy the weak formulation (11). However, we would like to write a similar formulation, yet with suitable corrective terms, that they satisfy. This idea was introduced in [VV03, Proposition 5.1] and exploited in MV07 to successfully obtain a $L^{1}$-error estimate for the upwind discretization of a transport problem in the whole space as well.

Lemma 4.3. For every test function $\varphi$ in $W^{1,1}([0, T] \times \bar{\Omega})$, we have

$$
\begin{gathered}
\iint_{\Omega^{T}} u_{\Delta x}\left(\partial_{t} \varphi+\mathbf{v} \cdot \nabla_{x} \varphi\right) d x d t+\int_{\Omega} u_{\Delta x}^{0} \varphi(0, .) d x-\int_{\Omega} u_{\Delta x}^{N} \varphi(T, .) d x \\
-\iint_{\Gamma^{T}}\left(\gamma u_{\Delta x}\right) \varphi \mathbf{v} \cdot \mathbf{n} d \sigma d s=\mu_{\Delta x}(\varphi)+\nu_{\Delta x}(\varphi),
\end{gathered}
$$


where the error terms in the weak formulation are defined by

$$
\mu_{\Delta x}(\varphi):=\sum_{\substack{0 \leq n<N \\ 0 \in \mathcal{T}}}|K|\left(u_{K}^{n+1}-u_{K}^{n}\right) f_{K^{n}}\left(\varphi(t, x)-\varphi\left(t^{n+1}, x\right)\right) d x d t
$$

and

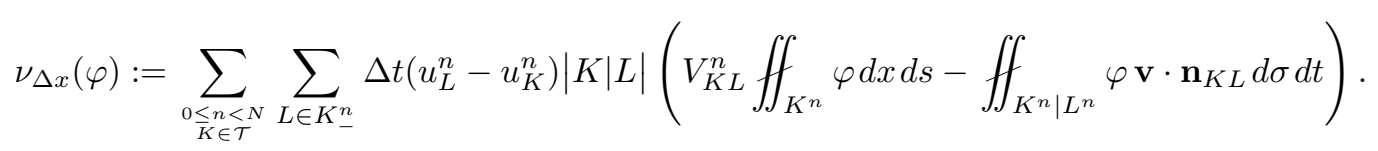

The proof is not detailed since it is very similar to the one in MV07 for instance, excepted that one needs to take care of boundary terms.

When $u^{0}=\mathbf{1}_{E^{0}}$ and $u_{b}=\mathbf{1}_{E_{b}}$ are indicatrix functions, we deduce from (29), that

$$
\left|u-u_{\Delta x}\right|=\left(u-u_{\Delta x}\right)(2 u-1) \quad\left(\text { resp. }\left|\gamma u-\gamma u_{\Delta x}\right|=\left(\gamma u-\gamma u_{\Delta x}\right)(2(\gamma u)-1)\right) .
$$

Therefore, if we subtract (30) and (11) and use the test function $\varphi=2 u-1$, which is also a solution of the transport equation, we formally obtain the following estimate

$$
E\left(u, u_{\Delta x}, T\right) \leq \int_{\Omega}\left|u^{0}-u_{\Delta x}^{0}\right| d x+\iint_{\Gamma_{\text {in }}^{T}}\left|u_{b}-\gamma u_{\Delta x}\right||\mathbf{v} \cdot \mathbf{n}| d \sigma d t+2\left|\mu_{\Delta x}(u)\right|+2\left|\nu_{\Delta x}(u)\right|,
$$

where we have used that $\mu_{\Delta x}(2 u-1)=2 \mu_{\Delta x}(u)$ and $\nu_{\Delta x}(2 u-1)=2 \nu_{\Delta x}(u)$.

However, this computation is not valid since $u$ is not regular enough to serve as a test function (it takes exactly two values 0 and 1 and thus does not belong to $\left.W^{1,1}\right)$.

The cure proposed in MV07] to deal with such a difficulty somehow consists in replacing the initial data $u^{0}$ by one of its regularization (there is no boundary data in this work). When Equation (1) is posed on the whole space $\mathbb{R}^{d}$, the solution inherits the regularity of the initial data. The authors propose a kind of smoothing of the initial indicatrix function $u^{0}$ by replacing, roughly speaking, the level set $E^{0}$ by some well-behaved approximation made of small cubes whose size is controlled all along the flow. Then, they use the regularity property of the flow to estimate the number of cells that are polluted by this regularization process on the time interval.

It seems that we cannot use the same strategy in the present work because of the influence of the boundary data and of the geometry of the inflow part of the boundary, as recalled in the introduction.

As a consequence, we choose here a slightly different approach that rather consists in regularizing the solution $u$ itself by using a kind of convolution operator in space. Such regularization ideas originate from the celebrated Friedrichs lemma and its application to the renormalized solutions theory for the transport equation (see [DL89, Boy05, BF13]). The value of the regularization parameter $\epsilon$ will be fixed at the end of the proof as a function of $\Delta x$.

To define the smoothing operator $\mathcal{S}_{\epsilon}$, we use a non-negative kernel $\zeta \in \mathcal{C}^{\infty}\left(\mathbb{R}^{d}\right)$, supported in the unit ball $B(0,1)$, and with integral 1 . For any locally integrable function $w \in L_{\text {loc }}^{1}(\Omega)$, and any $0<\epsilon<\xi_{\Omega} / 6$, we define

$$
\left(\mathcal{S}_{\epsilon} w\right)(x):=\int_{\Omega} w(y) \zeta\left(\frac{x-2 \epsilon \mathbf{n}(x)-y}{\epsilon}\right) \frac{d y}{\epsilon^{d}}=\int_{B(0,1)} w(x-2 \epsilon \mathbf{n}(x)-\epsilon y) \zeta(y) d y, \quad \text { for all } x \in \Omega .
$$

Here, $\mathbf{n}$ is the extension of the unit outward normal vector field to the whole domain defined in (7). The term $2 \epsilon \mathbf{n}(x)$ in the definition of $\mathcal{S}_{\epsilon} w$ is a small shift of the point $x$ away from the boundary that ensures that no value of $w$ outside of $\Omega$ is used in the second integral expression. Indeed, by using the definition of $\mathbf{n}$, in (7), and the condition $\epsilon<\xi_{\Omega} / 6$, we can check that $x-2 \epsilon \mathbf{n}(x)-\epsilon y \in \Omega$ for any $x \in \Omega$ and any $y \in B(0,1)$. Such a construction ensures that $\mathcal{S}_{\epsilon} w$ is smooth and is indeed an approximation of $w$ as $\epsilon \rightarrow 0$.

We will show below that, if $u$ is a solution to the transport problem, then its regularization is almost a solution to the transport problem up to an error term that we can estimate. This is the purpose of the following result. 
Proposition 4.4. Let $\mathbf{v}$ satisfy (4) and $u \in L^{\infty}((0, T) \times \Omega) \cap L^{1}(0, T ; \mathrm{BV}(\Omega))$ be any weak solution of the transport equation

$$
\partial_{t} u+\mathbf{v} \cdot \nabla u=0
$$

in the distribution sense. Then its space regularization $u_{\epsilon}(t,):.=\mathcal{S}_{\epsilon} u(t,$.$) defined as in (32)$, with $0<\epsilon<\xi_{\Omega} / 6$, solves the equation

$$
\partial_{t} u_{\epsilon}+\mathbf{v} \cdot \nabla u_{\epsilon}=g_{\epsilon}
$$

in the distribution sense, where $g_{\epsilon} \in L^{\infty}((0, T) \times \Omega)$ satisfies the bounds

$$
\begin{gathered}
\left\|g_{\epsilon}\right\|_{L^{\infty}\left(\Omega^{T}\right)} \leq C\|u\|_{L^{\infty}\left(\Omega^{T}\right)}, \\
\left\|g_{\epsilon}\right\|_{L^{1}\left(\Omega^{T}\right)} \leq C \epsilon\|u\|_{L^{1}(0, T ; \operatorname{BV}(\Omega))},
\end{gathered}
$$

for some constant $C$ independent of $u$ and $\epsilon$.

Moreover, $u_{\epsilon}$ is smooth and satisfies

$$
\begin{gathered}
\inf _{\Omega^{T}} u \leq \inf _{\Omega^{T}} u_{\epsilon}, \quad \sup _{\Omega^{T}} u_{\epsilon} \leq \sup _{\Omega^{T}} u, \quad \text { and } \quad\left\|u_{\epsilon}-u\right\|_{L^{\infty}\left(0, T ; L^{1}(\Omega)\right)} \leq C \epsilon\|u\|_{L^{1}(0, T ; \mathrm{BV}(\Omega))}, \\
\left\|\nabla_{t, x} u_{\epsilon}\right\|_{L^{1}\left(\Omega^{T}\right)} \leq C\|u\|_{L^{1}(0, T ; \operatorname{BV}(\Omega))}, \quad \text { and } \quad\left\|\nabla_{t, x} u_{\epsilon}\right\|_{L^{\infty}\left(\Omega^{T}\right)} \leq \frac{C\|u\|_{L^{\infty}\left(\Omega^{T}\right)}}{\epsilon}
\end{gathered}
$$

The next theorem gives an estimate on the error between the traces $\gamma u$ and $\gamma u_{\epsilon}$ (which is nothing but the restriction of $u_{\epsilon}$ to $\Gamma^{T}$ ) which is not given by the previous result.

Theorem 4.5. Assume that the regularity assumptions (2), (3) and (4) hold as well as (10). Let $u$ be the exact solution of the transport equation (1) and $u_{\epsilon}(t,):.=\mathcal{S}_{\epsilon} u(t,$.$) its regularization defined by (32). Then,$ there exists a $C>0$ depending only on $\Omega, T$ and $\mathbf{v}$, such that

$$
\iint_{\Gamma^{T}}\left|u_{\epsilon}-\gamma u\right||\mathbf{v} \cdot \mathbf{n}| d \sigma d t \leq C\left(\operatorname{TV}_{\Omega}\left(u^{0}\right)+\operatorname{TV}_{\Gamma^{T}}\left(u_{b}\right)+\operatorname{Per}_{\Gamma^{T}}\left(\Gamma_{\mathrm{in}}^{T}\right)+\|u\|_{L^{\infty}\left(\Omega^{T}\right)}\right) \epsilon .
$$

Those two results are proved in Section 5

With Proposition 4.4 and Theorem 4.5 in hands, we can use $2 u_{\epsilon}-1$ as a test function in 30 and obtain the following proposition.

Proposition 4.6. Suppose that $u^{0}=\mathbf{1}_{E^{0}}$ and $u_{b}=\mathbf{1}_{E_{b}}$ are indicatrix functions of finite perimeter sets. Suppose that assumption 10 holds. Then there exists a $C>0$ depending only on $\Omega, T$ and $\mathbf{v}$ such that

$$
\begin{aligned}
E\left(u, u_{\Delta x}, T\right) \leq & \int_{\Omega}\left|u^{0}-u_{\Delta x}^{0}\right|(x) d x+\iint_{\Gamma_{\text {in }}^{T}}\left|u_{b}-\gamma u_{\Delta x}\right||\mathbf{v} \cdot \mathbf{n}| d \sigma d t \\
& \quad+C \epsilon\left(1+\operatorname{Per}_{\Gamma^{T}}\left(\Gamma_{\text {in }}^{T}\right)+\operatorname{Per}_{\Gamma^{T}}\left(E_{b}\right)+\operatorname{Per}_{\Omega}\left(E^{0}\right)\right)+2\left|\mu_{\Delta x}\left(u_{\epsilon}\right)\right|+2\left|\nu_{\Delta x}\left(u_{\epsilon}\right)\right|
\end{aligned}
$$

Proof. We set $w=2 u-1$ and $w_{\epsilon}=2 u_{\epsilon}-1$. By subtracting the weak formulation for the exact solution (11) from the weak formulation for the approximate solution (30) and choosing $\varphi=w_{\epsilon}$, we obtain

$$
\begin{gathered}
\iint_{\Omega^{T}}\left(u_{\Delta x}-u\right)\left(\partial_{t} w_{\epsilon}+\mathbf{v} \cdot \nabla_{x} w_{\epsilon}\right) d x d t+\int_{\Omega}\left(u-u_{\Delta x}\right)(T, x) w_{\epsilon}(T, x) d x \\
+\iint_{\Gamma_{\text {out }}^{T}}\left(\gamma u-\gamma u_{\Delta x}\right) w_{\epsilon}|\mathbf{v} \cdot \mathbf{n}| d \sigma d t=\int_{\Omega}\left(u^{0}-u_{\Delta x}^{0}\right) w_{\epsilon}(0, x) d x \\
\quad+\iint_{\Gamma_{\text {in }}^{T}}\left(u_{b}-\gamma u_{\Delta x}\right) w_{\epsilon}|\mathbf{v} \cdot \mathbf{n}| d \sigma d t+\mu_{\Delta x}\left(w_{\epsilon}\right)+\nu_{\Delta x}\left(w_{\epsilon}\right) .
\end{gathered}
$$


Since $w_{\epsilon}$ solves the transport equation with right-hand side $2 g_{\epsilon}$ (see Proposition 4.4), we can write

$$
\begin{aligned}
& \int_{\Omega}\left(u-u_{\Delta x}\right)(T, x)(2 u-1)(T, x) d x+\iint_{\Gamma_{\text {out }}^{T}}\left(\gamma u-\gamma u_{\Delta x}\right)(2 \gamma u-1)|\mathbf{v} \cdot \mathbf{n}| d \sigma d t \\
& =2 \int_{\Omega}\left(u-u_{\Delta x}\right)(T, x)\left(u-u_{\epsilon}\right)(T, x) d x+2 \int_{\Gamma_{\text {out }}^{T}}\left(\gamma u-\gamma u_{\Delta x}\right)\left(\gamma u-u_{\epsilon}\right)|\mathbf{v} \cdot \mathbf{n}| d \sigma d t \\
& \quad-2 \iint_{\Omega^{T}}\left(u_{\Delta x}-u\right) g_{\epsilon} d x d t+\int_{\Omega}\left(u^{0}-u_{\Delta x}^{0}\right)\left(2 u_{\epsilon}-1\right)(0, x) d x \\
& \quad+\iint_{\Gamma_{\text {in }}^{T}}\left(u_{b}-\gamma u_{\Delta x}\right)\left(2 u_{\epsilon}-1\right) \mathbf{v} \cdot \mathbf{n} d \sigma d t+2 \mu_{\Delta x}\left(u_{\epsilon}\right)+2 \nu_{\Delta x}\left(u_{\epsilon}\right) .
\end{aligned}
$$

By using (31), and the fact that $2 u_{\epsilon}-1$ takes its values in $[-1,1]$, we get

$$
\begin{aligned}
\int_{\Omega}\left|u-u_{\Delta x}\right|(T, & x) d x+\iint_{\Gamma_{\text {out }}^{T}}\left|\gamma u-\gamma u_{\Delta x}\right||\mathbf{v} \cdot \mathbf{n}| d \sigma d t \\
\leq & 4\|u\|_{L^{\infty}\left(\Omega^{T}\right)} \int_{\Omega}\left|u-u_{\epsilon}\right|(T, x) d x+4\|u\|_{L^{\infty}\left(\Omega^{T}\right)} \iint_{\Gamma_{\text {out }}^{T}}\left|\gamma u-u_{\epsilon}\right||\mathbf{v} \cdot \mathbf{n}| d \sigma d t \\
& +4\|u\|_{L^{\infty}\left(\Omega^{T}\right)}\left\|g_{\epsilon}\right\|_{L^{1}\left(\Omega^{T}\right)}+\int_{\Omega}\left|u^{0}-u_{\Delta x}^{0}\right| d x+\iint_{\Gamma_{\text {in }}^{T}}\left|u_{b}-\gamma u_{\Delta x}\right||\mathbf{v} \cdot \mathbf{n}| d \sigma d t \\
& +2\left|\mu_{\Delta x}\left(u_{\epsilon}\right)\right|+2\left|\nu_{\Delta x}\left(u_{\epsilon}\right)\right| .
\end{aligned}
$$

Using Proposition 4.4 and Theorem 4.5, we get

$$
\begin{aligned}
E\left(u, u_{\Delta x}, T\right) \leq C\left(\operatorname{Per}_{\Omega}\left(E^{0}\right)\right. & \left.+\operatorname{Per}_{\Gamma^{T}}\left(E_{b}\right)+\operatorname{Per}_{\Gamma^{T}}\left(\Gamma_{\mathrm{in}}^{T}\right)+1\right) \epsilon+C \epsilon\|u\|_{L^{1}(0, T ; \mathrm{BV}(\Omega))} \\
& +\int_{\Omega}\left|u^{0}-u_{\Delta x}^{0}\right| d x+\iint_{\Gamma_{\mathrm{in}}^{T}}\left|u_{b}-\gamma u_{\Delta x}\right||\mathbf{v} \cdot \mathbf{n}| d \sigma d t+2\left|\mu_{\Delta x}\left(u_{\epsilon}\right)\right|+2\left|\nu_{\Delta x}\left(u_{\epsilon}\right)\right| .
\end{aligned}
$$

The result follows from Theorem 2.5

\subsection{Estimate of the data approximation errors and of the weak formulation errors}

We first analyze the size of the error terms related to the discretization of initial and boundary data.

Proposition 4.7. Under the same assumptions as in Theorem 4.1, there exists a $C>0$ depending only on $\Omega, T, \mathbf{v}$, and $\alpha$ such that

$$
\int_{\Omega}\left|u^{0}-u_{\Delta x}^{0}\right|(x) d x \leq C\left(\operatorname{TV}_{\Omega}\left(u^{0}\right)+\left\|u^{0}\right\|_{L^{\infty}(\Omega)}\right) \Delta x
$$

and

$$
\iint_{\Gamma_{\mathrm{in}}^{T}}\left|u_{b}-\gamma u_{\Delta x}\right||\mathbf{v} \cdot \mathbf{n}| d \sigma d t \leq C\left(\|u\|_{L^{\infty}\left(\Omega^{T}\right)}+\operatorname{TV}_{\Gamma^{T}}\left(u_{b}\right)\right) \sqrt{\Delta x}
$$

Note that the main reason for the bound in $\sqrt{\Delta x}$, instead of $\Delta x$, in the second inequality is the lack of regularity of $\mathbf{v}$. This result is proved in Section 6.1.

Using now the properties of the regularization $u_{\epsilon}$ we have already established, we can prove the following estimate for the error terms of the numerical weak formulation $\mu_{\Delta x}\left(u_{\epsilon}\right)$ and $\nu_{\Delta x}\left(u_{\epsilon}\right)$.

Proposition 4.8. Under the same assumptions as in Theorem 4.1, there exists a $C>0$ depending only on $\Omega, T, \alpha$ and $\lambda$, such that

$$
\left|\mu_{\Delta x}\left(u_{\epsilon}\right)\right|+\left|\nu_{\Delta x}\left(u_{\epsilon}\right)\right| \leq C\left(\sqrt{\mathcal{D}_{\Delta x}\left(u_{\Delta x}, T\right) \frac{\Delta x}{\epsilon}}+\frac{\Delta x}{\epsilon}\right) \sqrt{\|u\|_{L^{\infty}\left(\Omega^{T}\right)}} \sqrt{\|u\|_{L^{\infty}\left(\Omega^{T}\right)}+\|u\|_{L^{1}(0, T ; \mathrm{BV}(\Omega))}}
$$


The proof of this result is given in Section 6.2

We have already proved in (27) that $\mathcal{D}_{\Delta x}\left(u_{\Delta x}, T\right)$ is controlled by $\mathcal{E}_{\Delta x}\left(u_{\Delta x}, T\right)$. It appears from Proposition 4.8 that we need now to prove that $\mathcal{E}_{\Delta x}\left(u_{\Delta x}, T\right)$ is itself controlled by the $L^{1}$-error that we are interested in.

Proposition 4.9. The total $L^{2}$-energy balance term $\mathcal{E}_{\Delta x}\left(u_{\Delta x}, T\right)$ is controlled by the $L^{1}$-error at final time as follows

$$
\mathcal{E}_{\Delta x}\left(u_{\Delta x}, T\right) \leq 2\|u\|_{L^{\infty}\left(\Omega^{T}\right)} E\left(u, u_{\Delta x}, T\right) .
$$

Proof. Taking (13) into account, we rewrite $\mathcal{E}_{\Delta x}\left(u_{\Delta x}, T\right)$ as

$$
\mathcal{E}_{\Delta x}\left(u_{\Delta x}, T\right)=\int_{\Omega}\left(\left(u_{\Delta x}^{0}\right)^{2}-\left(u^{0}\right)^{2}\right)(x) d x-\int_{\Omega}\left(u_{\Delta x}^{2}-u^{2}\right)(T, x) d x-\iint_{\Gamma^{T}}\left(\gamma u_{\Delta x}^{2}-\gamma u^{2}\right)(\mathbf{v} \cdot \mathbf{n}) d \sigma d t
$$

By the Jensen inequality, the first term $\left\|u_{\Delta x}^{0}\right\|_{L^{2}}^{2}-\left\|u^{0}\right\|_{L^{2}}^{2}$ is non positive so that we immediately obtain

$$
\mathcal{E}_{\Delta x}\left(u_{\Delta x}, T\right) \leq 2\|u\|_{L^{\infty}\left(\Omega^{T}\right)} \int_{\Omega}\left|u_{\Delta x}-u\right|(T, x) d x+2\|u\|_{L^{\infty}\left(\Omega^{T}\right)} \iint_{\Gamma^{T}}\left|\gamma u_{\Delta x}-\gamma u\right||\mathbf{v} \cdot \mathbf{n}| d \sigma d t,
$$

by using the definition of $E\left(u, u_{\Delta x}, T\right)$.

\subsection{Conclusion of the proof of the main result}

We may now gather the results obtained so far to conclude the proof of Theorem 4.2 and consequently of Theorem 4.1 by the arguments developed in Section 4.1 .

Hence, let $u^{0}=\mathbf{1}_{E^{0}}$ and $u_{b}=\mathbf{1}_{E_{b}}, E^{0}$ and $E_{b}$ being sets of finite perimeter, and $\epsilon>0$ fixed.

- Propositions 4.6 and 4.7 give

$$
E\left(u, u_{\Delta x}, T\right) \leq C(\epsilon+\sqrt{\Delta x})\left(1+\operatorname{Per}_{\Gamma^{T}}\left(\Gamma_{\text {in }}^{T}\right)+\operatorname{Per}_{\Gamma^{T}}\left(E_{b}\right)+\operatorname{Per}_{\Omega}\left(E^{0}\right)\right)+2\left|\mu_{\Delta x}\left(u_{\epsilon}\right)\right|+2\left|\nu_{\Delta x}\left(u_{\epsilon}\right)\right|,
$$

since $\Delta x \leq \sqrt{\operatorname{diam}(\Omega)} \sqrt{\Delta x}$.

- We use now the dissipation estimate (27), Proposition 4.8 and the BV regularity result (Theorem 2.5) to get

$$
\begin{aligned}
E\left(u, u_{\Delta x}, T\right) \leq & C(\epsilon+\sqrt{\Delta x})\left(1+\operatorname{Per}_{\Gamma^{T}}\left(\Gamma_{\mathrm{in}}^{T}\right)+\operatorname{Per}_{\Gamma^{T}}\left(E_{b}\right)+\operatorname{Per}_{\Omega}\left(E^{0}\right)\right) \\
& +\frac{C}{\sqrt{\lambda}}\left(\sqrt{\mathcal{E}_{\Delta x}\left(u_{\Delta x}, T\right) \frac{\Delta x}{\epsilon}}+\frac{\Delta x}{\epsilon}\right) \sqrt{1+\operatorname{Per}_{\Gamma^{T}}\left(\Gamma_{\mathrm{in}}^{T}\right)+\operatorname{Per}_{\Gamma^{T}}\left(E_{b}\right)+\operatorname{Per}_{\Omega}\left(E^{0}\right) .}
\end{aligned}
$$

- Finally, with Proposition 4.9 we obtain

$$
\begin{aligned}
E\left(u, u_{\Delta x}, T\right) \leq & C(\epsilon+\sqrt{\Delta x})\left(1+\operatorname{Per}_{\Gamma^{T}}\left(\Gamma_{\mathrm{in}}^{T}\right)+\operatorname{Per}_{\Gamma^{T}}\left(E_{b}\right)+\operatorname{Per}_{\Omega}\left(E^{0}\right)\right) \\
& +\frac{C}{\sqrt{\lambda}}\left(\sqrt{E\left(u, u_{\Delta x}, T\right) \frac{\Delta x}{\epsilon}}+\frac{\Delta x}{\epsilon}\right) \sqrt{1+\operatorname{Per}_{\Gamma^{T}}\left(\Gamma_{\mathrm{in}}^{T}\right)+\operatorname{Per}_{\Gamma^{T}}\left(E_{b}\right)+\operatorname{Per}_{\Omega}\left(E^{0}\right)} .
\end{aligned}
$$

- We use now the Young inequality to deduce a bound on $E\left(u, u_{\Delta x}, T\right)$ from the previous inequality

$$
E\left(u, u_{\Delta x}, T\right) \leq C\left(\epsilon+\sqrt{\Delta x}+\frac{\Delta x}{\epsilon}\right)\left(1+\operatorname{Per}_{\Gamma^{T}}\left(\Gamma_{\text {in }}^{T}\right)+\operatorname{Per}_{\Gamma^{T}}\left(E_{b}\right)+\operatorname{Per}_{\Omega}\left(E^{0}\right)\right) .
$$

It remains now to choose $\epsilon=\sqrt{\Delta x}$ to get the final estimate

$$
E\left(u, u_{\Delta x}, T\right) \leq C\left(1+\operatorname{Per}_{\Gamma^{T}}\left(\Gamma_{\text {in }}^{T}\right)+\operatorname{Per}_{\Gamma^{T}}\left(E_{b}\right)+\operatorname{Per}_{\Omega}\left(E^{0}\right)\right) \sqrt{\Delta x} .
$$




\section{Intermediate results concerning the regularization of the solution}

This section is devoted to the proof of some intermediate results we used on the exact solution of the transport equation, namely Proposition 2.4. Proposition 4.4 and Theorem 4.5 .

\subsection{Proof of Proposition 4.4}

Let us first prove the following lemma.

Lemma 5.1. There exists a $C>0$ depending only on $\Omega$, such that for any $w \in \mathrm{BV}(\Omega)$ and any $0<\epsilon<\xi_{\Omega} / 6$ we have the estimate

$$
\int_{\Omega} \int_{B(0,1)}|w(x-2 \epsilon \mathbf{n}(x)-\epsilon y)-w(x)| d y d x \leq C \epsilon \operatorname{TV}_{\Omega}(w) .
$$

Proof. Let us first assume that $w \in \mathcal{C}^{\infty}(\bar{\Omega})$ and that $\epsilon$ is small enough so that $2 \epsilon\left\|D_{x} \mathbf{n}\right\|_{L^{\infty}(\Omega)}<1$. We can use Fubini's theorem and a change of variable to obtain

$$
\begin{aligned}
\int_{\Omega} \int_{B(0,1)}|w(x-2 \epsilon \mathbf{n}(x)-\epsilon y)-w(x)| d y d x & \leq \int_{\Omega} \int_{B(0,1)} \int_{0}^{1}|\nabla w(x-s \epsilon(2 \mathbf{n}(x)+y)) \cdot \epsilon(2 \mathbf{n}(x)+y)| d s d y d x \\
& =\int_{B(0,1)} \int_{0}^{1} \int_{\Omega}|\nabla w(x-s \epsilon(2 \mathbf{n}(x)+y)) \cdot \epsilon(2 \mathbf{n}(x)+y)| d x d s d y \\
& \leq \epsilon \frac{2\|\mathbf{n}\|_{L^{\infty}(\Omega)}+1}{1-2 \epsilon\left\|D_{x} \mathbf{n}\right\|_{L^{\infty}(\Omega)}} \int_{B(0,1)} \int_{0}^{1} \int_{\Omega}|\nabla w(x)| d x d s d y \\
& \leq C \epsilon \int_{\Omega}|\nabla w(x)| d x
\end{aligned}
$$

The claim follows by weak density of the set of smooth functions in BV $(\Omega)$.

We can now prove the proposition.

Proof of Proposition 4.4. The fact that $u_{\epsilon}$ solves a transport-like equation is a rather standard computation (see for instance, the details in [Boy05]) and we simply give here the resulting source term

$$
\begin{gathered}
g_{\epsilon}(t, x)=\int_{B(0,1)} u(t, x-2 \epsilon \mathbf{n}(x)-\epsilon y) \frac{\mathbf{v}(t, x)-\mathbf{v}(t, x-2 \epsilon \mathbf{n}(x)-\epsilon y)}{\epsilon} \cdot \nabla \zeta(y) d y \\
-2 \int_{B(0,1)} u(t, x-2 \epsilon \mathbf{n}(x)-\epsilon y) \mathbf{v}(t, x) \cdot D_{x} \mathbf{n}(x) \nabla \zeta(y) d y .
\end{gathered}
$$

The $L^{\infty}$-bound on $g_{\epsilon}$ immediately follows from the assumptions (4) on $\mathbf{v}$.

Observe now, by integration by parts with respect to the variable $y$, that we have

$$
\int_{B(0,1)} u(t, x) \frac{\mathbf{v}(t, x)-\mathbf{v}(t, x-2 \epsilon \mathbf{n}(x)-\epsilon y)}{\epsilon} \cdot \nabla \zeta(y) d y=\frac{1}{\epsilon} \int_{B(0,1)} u(t, x)(\operatorname{div} \mathbf{v})(t, x-2 \epsilon \mathbf{n}(x)-\epsilon y) \zeta(y) d y=0,
$$

since $\mathbf{v}$ is divergence free. Hence, we can rewrite $g_{\epsilon}$ as

$$
\begin{aligned}
g_{\epsilon}(t, x)= & \int_{B(0,1)}[u(t, x-2 \epsilon \mathbf{n}(x)-\epsilon y)-u(t, x)] \frac{\mathbf{v}(t, x)-\mathbf{v}(t, x-2 \epsilon \mathbf{n}(x)-\epsilon y)}{\epsilon} \cdot \nabla \zeta(y) d y \\
& -\int_{B(0,1)}[u(t, x-2 \epsilon \mathbf{n}(x)-\epsilon y)-u(t, x)] \mathbf{v}(t, x) \cdot D_{x} \mathbf{n}(x) \nabla \zeta(y) d y
\end{aligned}
$$

By using the assumption on $\mathbf{v}$ and $\mathbf{n}$, and 35 we immediately deduce

$$
\left\|g_{\epsilon}\right\|_{L^{1}\left(\Omega^{T}\right)} \leq C \epsilon \int_{0}^{T} \operatorname{TV}_{\Omega}(u(t, .)) d t \leq C \epsilon\|u\|_{L^{1}(0, T ; \mathrm{BV}(\Omega))} .
$$


The estimate on $\left\|u_{\epsilon}-u\right\|_{L^{\infty}\left(0, T ; L^{1}(\Omega)\right)}$ is straightforward from Lemma 5.1 , and the $L^{\infty}$-bound on $u_{\epsilon}$ comes from the definition 32 . Using similar computations as before, we find that

$$
\nabla_{x} u_{\epsilon}(t, x)=\int_{B(0,1)} \frac{u(t, x-2 \epsilon \mathbf{n}(x)-\epsilon y)-u(t, x)}{\epsilon} \nabla \zeta d y-2 \int_{B(0,1)} u(t, x-2 \epsilon \mathbf{n}(x)-\epsilon y) D_{x} \mathbf{n} \nabla \zeta d y
$$

and thus, by 35 , we get

$$
\left\|\nabla_{x} u_{\epsilon}\right\|_{L^{1}((0, T) \times \Omega)} \leq C\|u\|_{L^{1}(0, T ; \operatorname{BV}(\Omega))}, \quad \text { and }\left\|\nabla_{x} u_{\epsilon}\right\|_{L^{\infty}((0, T) \times \Omega)} \leq C \frac{\|u\|_{L^{\infty}((0, T) \times \Omega)}}{\epsilon} .
$$

The estimates on the time derivatives of $u_{\epsilon}$ simply follow from the equality $\partial_{t} u_{\epsilon}=g_{\epsilon}-\mathbf{v} \cdot \nabla_{x} u_{\epsilon}$, and the bounds on $g_{\epsilon}$ and $\nabla_{x} u_{\epsilon}$ we just obtained.

\subsection{Proofs of Proposition 2.4 and Theorem 4.5}

We shall actually need a slightly more general result than the one in Proposition 2.4 that we state below. It will be useful in the proof of Theorem 4.5 when we will need to analyze the trace of the regularization by convolution of the solution of the transport equation.

Proposition 5.2. Under the same assumptions as in Proposition 2.4, there exists a $C>0$ depending only on $\Omega, T$ and $\mathbf{v}$ such that we have the following estimate for any $y \in B(0,1)$, and any $\xi \in\left(0, \xi_{\Omega}\right)$,

$$
\iint_{\Gamma_{\mathrm{in}}^{T}}\left|u_{b}(t, \tilde{x})-u\left(t, \tilde{x}-\xi \mathbf{n}(\tilde{x})+\frac{\xi}{2} y\right)\right||\mathbf{v}(t, \tilde{x}) \cdot \mathbf{n}(\tilde{x})| d \sigma(\tilde{x}) d t \leq C\left(\operatorname{Per}_{\Gamma^{T}}\left(\Gamma_{\mathrm{in}}^{T}\right)+\operatorname{TV}_{\Gamma^{T}}\left(u_{b}\right)+\|u\|_{L^{\infty}}\right) \xi .
$$

Proposition 2.4 is nothing but the case $y=0$ of the previous result.

Proof. By the arguments exposed in Section 4.1, we see that we only need to deal with the case where $u^{0}=\mathbf{1}_{E^{0}}$ and $u_{b}=\mathbf{1}_{E_{b}}$ for some finite perimeter sets $E^{0} \subset \Omega$ and $E_{b} \subset \Gamma^{T}$, so that in particular we have the bounds 29.

- We first prove that, for any $E \subset \Gamma^{T}$ of finite perimeter and any $\xi<2 \xi_{\Omega} / 3$, we have

$$
\left|\iint_{E}\left(\gamma u(t, \tilde{x})-u\left(t, \tilde{x}-\xi \mathbf{n}(\tilde{x})+\frac{\xi}{2} y\right)\right)(\mathbf{v}(t, \tilde{x}) \cdot \mathbf{n}(\tilde{x})) d \sigma(\tilde{x}) d t\right| \leq C \xi\left(1+\operatorname{Per}_{\Gamma^{T}} E\right) .
$$

Indeed, let $\left(f_{n}\right)_{n}$ a sequence of smooth functions on $\Gamma^{T}$ that approximate $\mathbf{1}_{E}$ as in (6).

Since $\mathbf{n}$ is a unitary vector field, we easily see that, for any $y \in B(0,1)$, the map

$$
\Phi_{y}:(s, \tilde{x}) \in\left[0,2 \xi_{\Omega} / 3\right] \times \Gamma \mapsto \tilde{x}-s \mathbf{n}(\tilde{x})+(s / 2) y,
$$

is one to one into a subset of $\mathcal{O}_{\xi_{\Omega}}$ and that

$$
\left\|\nabla \Phi_{y}\right\|_{L^{\infty}\left(\left[0,2 \xi_{\Omega} / 3\right] \times \Gamma\right)}+\left\|\nabla\left(\Phi_{y}\right)^{-1}\right\|_{L^{\infty}\left(\operatorname{Im}\left(\Phi_{y}\right)\right)} \leq C,
$$

for some $C$ independent of $y$. Observe that

$$
\Phi_{y}(\{\xi\} \times \Gamma)=\Gamma_{\xi}+(\xi / 2) y,
$$

which is just a translation of $\Gamma_{\xi}$, so that in particular the normal field to the manifold $\Gamma_{\xi}+(\xi / 2) y$ is the translation of the normal field to $\Gamma_{\xi}$.

We also define the open set

$$
\mathcal{O}_{\xi, y}:=\Phi_{y}((0, \xi) \times \Gamma),
$$

that satisfies

$$
\mathcal{O}_{\xi, y} \subset \mathcal{O}_{3 / 2 \xi} .
$$




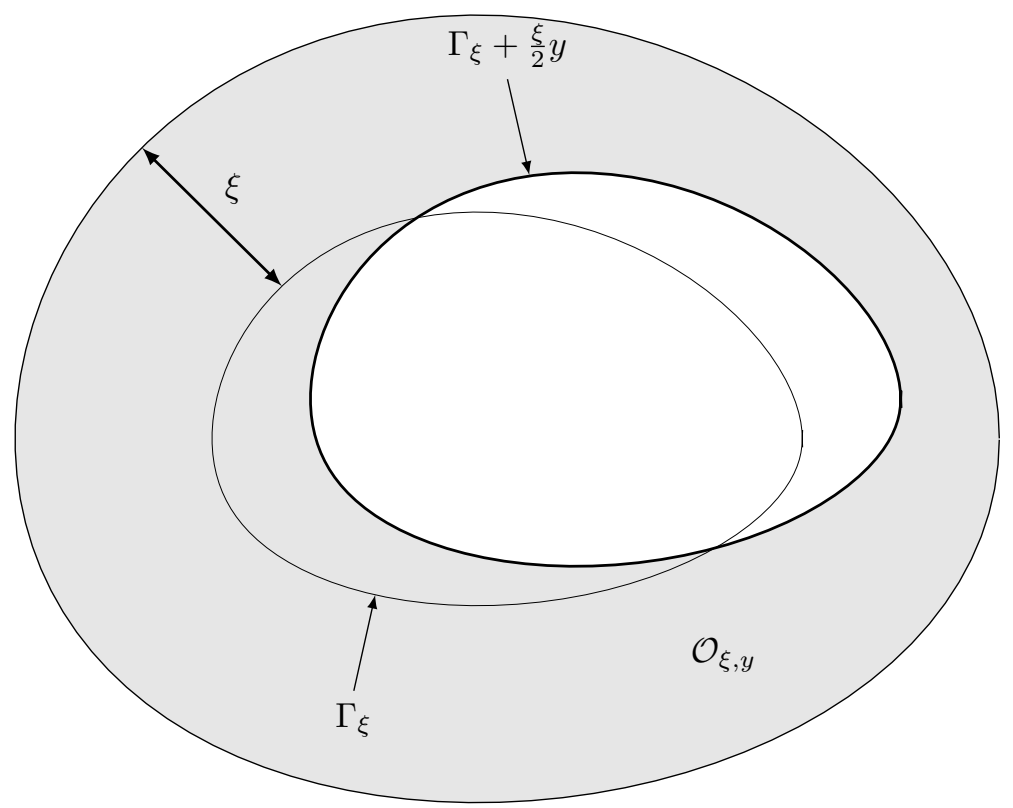

Figure 4: Definition of $\mathcal{O}_{\xi, y}$ for some $\xi$ and $y$

We introduce a test function $\varphi$ defined in $(0, T) \times \overline{\mathcal{O}_{\xi, y}}$ as follows (and extended by 0 on the whole domain $[0, T] \times \Omega)$

$$
\varphi(t, \tilde{x}-s \mathbf{n}(\tilde{x})+(s / 2) y)=f_{n}(t, \tilde{x}), \quad \forall s \in[0, \xi], \forall(t, \tilde{x}) \in \Gamma^{T} .
$$

Using this test function in (11) is possible and makes appear a boundary term on $\Gamma_{\xi, y}$. More precisely we obtain the following formula (see Boy05 for similar computations)

$$
\begin{aligned}
\iint_{\Gamma^{T}} \varphi(t, \tilde{x}) \gamma u(t, \tilde{x}) & (\mathbf{v}(t, \tilde{x}) \cdot \mathbf{n}(\tilde{x})) d \sigma(\tilde{x}) d t \\
& -\int_{0}^{T} \int_{\Gamma_{\xi}+(\xi / 2) y} \varphi(t, x) u(t, x)\left(\mathbf{v}(t, x) \cdot \mathbf{n}_{\Gamma_{\xi}+(\epsilon / 2) y}(x)\right) d \sigma(x) d t \\
= & -\int_{\mathcal{O}_{\xi, y}} u^{0} \varphi(0, .) d x+\int_{\mathcal{O}_{\xi, y}} u(T, .) \varphi(T, .) d x-\int_{0}^{T} \int_{\mathcal{O}_{\xi, y}} u\left(\partial_{t} \varphi+\mathbf{v} \cdot \nabla \varphi\right) d x d t .
\end{aligned}
$$

- By definition of $\varphi$, the first integral in (37) just reads

$$
\iint_{\Gamma^{T}} f_{n}(t, \tilde{x}) \gamma u(t, \tilde{x})(\mathbf{v}(t, \tilde{x}) \cdot \mathbf{n}(\tilde{x})) d \sigma(\tilde{x}) d t,
$$

whereas the second one can be expressed through the affine change of variable $x \mapsto x-\frac{\xi}{2} y$ as follows

$$
\int_{0}^{T} \int_{\Gamma_{\xi}} f_{n}\left(t, P_{\Gamma} x\right) u\left(t, x+\frac{\xi}{2} y\right)\left(\mathbf{v}\left(t, x+\frac{\xi}{2} y\right) \cdot \mathbf{n}(x)\right) d \sigma(x) d t .
$$

Using now the change of variable (8) in this integral leads to the expression

$$
\iint_{\Gamma^{T}} f_{n}(t, \tilde{x}) u\left(t, \tilde{x}-\xi \mathbf{n}(\tilde{x})+\frac{\xi}{2} y\right) J(\xi, \tilde{x})\left(\mathbf{v}\left(t, \tilde{x}-\xi \mathbf{n}(\tilde{x})+\frac{\xi}{2} y\right) \cdot \mathbf{n}(\tilde{x})\right) d \sigma(\tilde{x}) d t .
$$

- The first two terms in the right-hand side of 37 can simply be bounded, since $\|\varphi\|_{L^{\infty}\left(\Omega^{T}\right)} \leq 2$, as follows

$$
\left|\int_{\mathcal{O}_{\xi, y}} u^{0} \varphi(0, .) d x\right|+\left|\int_{\mathcal{O}_{\xi, y}} u(T, .) \varphi(T, .) d x\right| \leq 4\left|\mathcal{O}_{\xi, y}\right| \leq 4\left|\mathcal{O}_{3 \xi / 2}\right| \leq 6 \xi|\Gamma||| J \|_{L^{\infty}\left(\left(0, \xi_{\Omega}\right) \times \Gamma\right)} .
$$


- The last term in 37 is estimated as follows

$$
\begin{aligned}
\left|\int_{0}^{T} \int_{\mathcal{O}_{\xi, y}} u\left(\partial_{t} \varphi+\mathbf{v} \cdot \nabla \varphi\right) d x d t\right| & \leq\left(1+\|\mathbf{v}\|_{L^{\infty}\left(\Omega^{T}\right)}\right) \int_{0}^{T} \int_{\mathcal{O}_{\xi, y}}\left|\nabla_{t, x} \varphi\right| d x d t \\
& \leq\left(1+\|\mathbf{v}\|_{L^{\infty}\left(\Omega^{T}\right)}\right) \int_{0}^{T} \int_{\mathcal{O}_{3 \xi / 2}}\left|\nabla_{t, x} \varphi\right| d x d t
\end{aligned}
$$

Using the definition of $\varphi$, the properties of $\Phi_{y}$ and the change of variable (8), we obtain that this integral is bounded by

$$
\frac{3}{2} \xi\left(1+\|\mathbf{v}\|_{L^{\infty}\left(\Omega^{T}\right)}\right)\|J\|_{L^{\infty}\left(\left[0, \xi_{\Omega}\right] \times \Gamma\right)}\left\|\nabla \Phi_{y}^{-1}\right\|_{L^{\infty}\left(\operatorname{Im}\left(\Phi_{y}\right)\right)} \iint_{\Gamma^{T}}\left|\nabla_{t, \tilde{x}} f_{n}\right| d \sigma(\tilde{x}) d t .
$$

Gathering all those estimates, using that $J(0, \tilde{x})=1$ for any $\tilde{x} \in \Gamma$ and the fact that

$$
\left|\left(\mathbf{v}\left(t, \tilde{x}-\xi \mathbf{n}(\tilde{x})+\frac{\xi}{2} y\right) \cdot \mathbf{n}(\tilde{x})\right) J(\xi, \tilde{x})-(\mathbf{v}(t, \tilde{x}) \cdot \mathbf{n}(\tilde{x}))\right| \leq\|\mathbf{v}\|_{L^{\infty}\left(\Omega^{T}\right)} L_{J} \xi+\frac{3}{2} \xi L_{\mathbf{v}},
$$

we finally obtain the bound

$$
\begin{aligned}
& \left|\iint_{\Gamma^{T}} f_{n}(t, \tilde{x})\left(\gamma u(t, \tilde{x})-u\left(t, \tilde{x}-\xi \mathbf{n}(\tilde{x})+\frac{\xi}{2} y\right)\right)(\mathbf{v}(t, \tilde{x}) \cdot \mathbf{n}(\tilde{x})) d \sigma(\tilde{x}) d t\right| \\
\leq & C\left(\|\mathbf{v}\|_{L^{\infty}\left(\Omega^{T}\right)} L_{J}+L_{\mathbf{v}}\right) \xi+C\|J\|_{L^{\infty}\left(\left[0, \xi_{\Omega}\right] \times \Gamma\right)}|\Gamma| \xi+C\left(1+\|\mathbf{v}\|_{L^{\infty}\left(\Omega^{T}\right)}\right)\|J\|_{L^{\infty}\left(\left[0, \xi_{\Omega}\right] \times \Gamma\right)} \xi\left(\iint_{\Gamma^{T}}\left|\nabla_{t, \tilde{x}} f_{n}\right| d \sigma(\tilde{x}) d t\right) .
\end{aligned}
$$

Passing to the limit as $n \rightarrow \infty$, we obtain

$$
\left|\iint_{E}\left(\gamma u(t, \tilde{x})-u\left(t, \tilde{x}-\xi \mathbf{n}(\tilde{x})+\frac{\xi}{2} y\right)\right)(\mathbf{v}(t, \tilde{x}) \cdot \mathbf{n}(\tilde{x})) d \sigma(\tilde{x}) d t\right| \leq C \xi\left(1+\operatorname{Per}_{\Gamma^{T}}(E)\right),
$$

where $C$ depends only on $\mathbf{v}$ and $\Omega$.

- We first apply 36 to the set $E^{+}=\Gamma_{\text {in }}^{T} \cap E_{b}$. On this subset of $\Gamma^{T}$, we have $\gamma u=u_{b}=1$ so that we have

$$
\left|\iint_{E^{+}}\left(1-u\left(t, \tilde{x}-\xi \mathbf{n}(\tilde{x})+\frac{\xi}{2} y\right)\right)(\mathbf{v}(t, \tilde{x}) \cdot \mathbf{n}(\tilde{x})) d \sigma(\tilde{x}) d t\right| \leq C \xi\left(1+\operatorname{Per}_{\Gamma^{T}}\left(E^{+}\right)\right),
$$

and since the function under the integral is non-positive almost everywhere (because $u \leq 1$ and $\mathbf{v} \cdot \mathbf{n}<0$ on $\Gamma_{\text {in }}^{T}$ ), we finally proved that

$$
\iint_{E^{+}}\left|u_{b}(t, \tilde{x})-u\left(t, \tilde{x}-\xi \mathbf{n}(\tilde{x})+\frac{\xi}{2} y\right)\right||\mathbf{v}(t, \tilde{x}) \cdot \mathbf{n}(\tilde{x})| d \sigma(\tilde{x}) d t \leq C \xi\left(1+\operatorname{Per}_{\Gamma^{T}}\left(E^{+}\right)\right) .
$$

- Similarly, we apply 36 to the set $E^{-}=\Gamma_{\text {in }}^{T} \cap\left(\Gamma^{T} \backslash E_{b}\right)$. On this subset of $\Gamma^{T}$ we have $\gamma u=u_{b}=0$ so that we deduce

$$
\left|\iint_{E^{-}}\left(0-u\left(t, \tilde{x}-\xi \mathbf{n}(\tilde{x})+\frac{\xi}{2} y\right)\right)(\mathbf{v}(t, \tilde{x}) \cdot \mathbf{n}(\tilde{x})) d \sigma(\tilde{x}) d t\right| \leq C \xi\left(1+\operatorname{Per}_{\Gamma^{T}}\left(E^{-}\right)\right),
$$

and since the function we integrate is of constant sign, we deduce that

$$
\iint_{E^{-}}\left|u_{b}(t, \tilde{x})-u\left(t, \tilde{x}-\xi \mathbf{n}(\tilde{x})+\frac{\xi}{2} y\right)\right||\mathbf{v}(t, \tilde{x}) \cdot \mathbf{n}(\tilde{x})| d \sigma(\tilde{x}) d t \leq C \xi\left(1+\operatorname{Per}_{\Gamma^{T}}\left(E^{-}\right)\right) .
$$

- Adding the previous two inequalities and using that $\operatorname{Per}_{\Gamma^{T}}(A \cap B) \leq \operatorname{Per}_{\Gamma^{T}}(A)+\operatorname{Per}_{\Gamma^{T}}(B)$ and that $\operatorname{Per}_{\Gamma^{T}}\left(\Gamma^{T} \backslash A\right)=\operatorname{Per}_{\Gamma^{T}}(A)$, we finally obtain

$$
\iint_{\Gamma_{\text {in }}^{T}}\left|u_{b}(t, \tilde{x})-u\left(t, \tilde{x}-\xi \mathbf{n}(\tilde{x})+\frac{\xi}{2} y\right)\right||\mathbf{v}(t, \tilde{x}) \cdot \mathbf{n}(\tilde{x})| d \sigma(\tilde{x}) d t \leq C \xi\left(1+\operatorname{Per}_{\Gamma^{T}}\left(\Gamma_{\text {in }}^{T}\right)+\operatorname{Per}_{\Gamma^{T}}\left(E_{b}\right)\right) .
$$


We can now easily prove the error estimates between the traces $\gamma u$ and $\gamma u_{\epsilon}$.

Proof of Theorem 4.5. The estimate for the inflow boundary simply comes by integration of the inequality in Proposition 5.2 with respect to $y$ after multiplication by the kernel $\zeta(y)$ as follows

$$
\begin{aligned}
& \iint_{\Gamma_{\mathrm{in}}^{T}} \mid u_{b}(t, \tilde{x})- u_{\epsilon}(t, \tilde{x})|| \mathbf{v}(t, \tilde{x}) \cdot \mathbf{n}(\tilde{x}) \mid d \sigma(\tilde{x}) d t \\
& \leq \int_{B(0,1)} \iint_{\Gamma_{\mathrm{in}}^{T}} \zeta(y)\left|u_{b}(t, \tilde{x})-u(t, \tilde{x}-2 \epsilon \mathbf{n}(\tilde{x})+\epsilon y)\right||\mathbf{v}(t, \tilde{x}) \cdot \mathbf{n}(\tilde{x})| d \sigma(\tilde{x}) d t d y \\
& \leq C\left(\operatorname{Per}_{\Gamma^{T}}\left(\Gamma_{\mathrm{in}}^{T}\right)+\operatorname{TV}_{\Gamma^{T}}\left(u_{b}\right)+\|u\|_{L^{\infty}\left(\Omega^{T}\right)}\right) \epsilon .
\end{aligned}
$$

To obtain the estimate on the outflow boundary terms, we simply write the equation satisfied by $\left|u_{\epsilon}-u\right|$ (using the renormalization property in Theorem 2.2 .

$$
\partial_{t}\left|u_{\epsilon}-u\right|+\mathbf{v} \cdot \partial_{x}\left|u_{\epsilon}-u\right|=\operatorname{sign}\left(u_{\epsilon}-u\right) g_{\epsilon},
$$

and we use the test function $\varphi=1$ on this equation to obtain

$$
\begin{aligned}
\int_{\Omega}\left|u_{\epsilon}-u\right|(T, .) d x+\iint_{\Gamma_{\text {out }}^{T}} \mid & u_{\epsilon}-\gamma u|| \mathbf{v} \cdot \mathbf{n} \mid d \sigma d t \\
& =\int_{\Omega}\left|u_{\epsilon}-u\right|(0, .) d x+\iint_{\Gamma_{\text {in }}^{T}}\left|u_{\epsilon}-\gamma u\right||\mathbf{v} \cdot \mathbf{n}| d \sigma d t+\iint_{\Omega^{T}} \operatorname{sign}\left(u_{\epsilon}-u\right) g_{\epsilon} d x d t .
\end{aligned}
$$

Proposition 4.4 and the BV regularity property (Theorem 2.5) then gives

$$
\begin{aligned}
\iint_{\Gamma_{\text {out }}^{T}}\left|u_{\epsilon}-\gamma u\right||\mathbf{v} \cdot \mathbf{n}| d \sigma d t & \leq C\left(\operatorname{Per}_{\Gamma^{T}}\left(\Gamma_{\text {in }}^{T}\right)+\operatorname{TV}_{\Gamma^{T}}\left(u_{b}\right)+\|u\|_{L^{\infty}\left(\Omega^{T}\right)}\right) \epsilon+C \epsilon\|u\|_{L^{1}(0, T ; \operatorname{BV}(\Omega))} \\
& \leq C\left(\operatorname{TV}_{\Omega}\left(u^{0}\right)+\operatorname{TV}_{\Gamma^{T}}\left(u_{b}\right)+\operatorname{Per}_{\Gamma^{T}}\left(\Gamma_{\text {in }}^{T}\right)+\|u\|_{L^{\infty}\left(\Omega^{T}\right)}\right) \epsilon
\end{aligned}
$$

\section{Intermediate results on the numerical scheme}

\subsection{Proof of Proposition 4.7}

We start by proving the following approximation result.

Lemma 6.1. Let $f \in \mathrm{BV}\left(\Omega^{T}\right) \cap L^{\infty}\left(0, T,\left(W^{1, \infty}(\Omega)\right)\right)$, and denote its Lipschitz constant by

$$
L_{f}:=\sup _{t \in[0, T]}\left\|\nabla_{x} f(t, \cdot)\right\|_{L^{\infty}(\Omega)} .
$$

Consider a mesh of $\Omega$ and a time step $\Delta t$ as described in Section 3 and satisfying (15)-(17) and (19). For any $n$ and any $K \mid L \in \partial \mathcal{T}$, we set

$$
f_{K L}^{n}:=f_{K^{n} \mid L^{n}} f d \sigma d t
$$

1. For any $\xi<\xi_{\Omega}$ we have the estimate

$$
\sum_{\substack{0 \leq n<N \\ K \mid L \in \partial \mathcal{T}^{\mathrm{ext}}}} \iint_{K^{n} \mid L^{n}}\left|f-f_{K L}^{n}\right| d \sigma d t \leq C L_{f}\left|\Gamma^{T}\right|(\Delta x+\xi)+C \frac{\Delta x}{\xi} \operatorname{TV}_{(0, T) \times \mathcal{O}_{\xi}}(f) .
$$


In particular, with $\xi=\sqrt{\Delta x}$ and $(14)$, we have

$$
\sum_{\substack{0 \leq n<N \\ K \mid L \in \mathcal{L} \text { ext }}} \iint_{K^{n} \mid L^{n}}\left|f-f_{K L}^{n}\right| d \sigma d t \leq C \sqrt{\Delta x}
$$

with $C$ depending only on the assumed regularity of $f, \Omega$ and the mesh regularity parameter $\alpha$.

2. We have the following uniform bound

$$
\sum_{\substack{0 \leq n<N \\ K \mid L \in \partial \mathcal{T}}} \iint_{K^{n} \mid L^{n}}\left|f-f_{K L}^{n}\right| d \sigma d t \leq C
$$

Remark 6.2. If the trace of $f$ on $\Gamma^{T}$ were in the space $\mathrm{BV}\left(\Gamma^{T}\right)$ then, with some additional regularity assumptions on the mesh, we would have been able to improve the estimate $(38)$ by a bound in $O(\Delta x)$. However in general, with the assumptions we made on $f$, we want to emphasize that the BV regularity of the trace does not hold.

Consider the following example with $T=1 / 2, \Omega=(0,1):$ Let $\phi: \mathbb{R} \rightarrow \mathbb{R}$ be a smooth function such that $\phi(0)=1$ and $\operatorname{Supp}(\phi) \subset(-1,1)$ and define $f: \Omega^{T} \rightarrow \mathbb{R}$ as follows

$$
f(t, x)=\frac{\sin (\log t)}{\log t} \phi(x \log t), \quad \forall(t, x) \in(0,1 / 2) \times(0,1) .
$$

It is clear that $f$ is continuous on $\overline{\Omega^{T}}$, smooth in $\Omega^{T}$ and that

$$
\left|\partial_{x} f(t, x)\right|=\left|\sin (\log t)\left\|\phi^{\prime}(x \log t) \mid \leq\right\| \phi^{\prime} \|_{L^{\infty}},\right.
$$

so that $f$ is uniformly Lipschitz continuous with respect to $x$. Then we can compute

$$
\partial_{t} f(t, x)=-\frac{\sin (\log t)}{t(\log t)^{2}} \phi(x \log t)+\frac{\cos (\log t)}{t \log t} \phi(x \log t)+x \sin (\log t) \phi^{\prime}(x \log t) .
$$

It follows that

$$
\int_{\Omega^{T}}\left|\partial_{t} f\right| d x d t=\int_{0}^{1 / 2} \int_{0}^{1}\left|\partial_{t} f\right| d x d t \leq\left\|\phi^{\prime}\right\|_{L^{\infty}}+\|\phi\|_{L^{1}}\left(\int_{0}^{1 / 2} \frac{1}{t(\log t)^{3}}+\frac{1}{t|\log t|^{2}} d t\right)<+\infty
$$

so that $f \in \operatorname{BV}\left(\Omega^{T}\right)$. However, when one takes the trace of $f$ at $x=0$, we have

$$
\int_{\Gamma^{T}}\left|\partial_{t} f\right| d t \geq \int_{0}^{1 / 2}\left|\partial_{t} f(t, 0)\right| d t \geq \int_{0}^{1 / 2} \frac{|\cos (\log t)|}{t|\log t|} d t-\int_{0}^{1 / 2} \frac{1}{t(\log t)^{2}} d t=+\infty
$$

which proves that the trace of $f$ does not belong to $\mathrm{BV}\left(\Gamma^{T}\right)$.

Proof of Lemma 6.1. As usual, the proof is given for a smooth function $f$ and the claim is finally obtained by a standard density argument. For a given $n$ and a given $\sigma=K \mid L \in \partial \mathcal{T}$, we set $\sigma^{n}=\left(t^{n}, t^{n+1}\right) \times \sigma$. Moreover, we shall use the notation

$$
\mathcal{T}_{n, \sigma}:=\iint_{\sigma^{n}}\left|f-\int_{\sigma^{n}} f\right| d \sigma d t .
$$

1. Here, we only consider exterior faces $\sigma \in \partial \mathcal{T}^{\text {ext }}$. With any such face we associate a volume $K_{\xi, \sigma}$ (that has nothing to do with the mesh cells, see Fig. 5p defined through tangential and normal coordinates as follows

$$
K_{\xi, \sigma}:=\{\tilde{x}-s \mathbf{n}(\tilde{x}), \tilde{x} \in \sigma, s \in(0, \xi)\} .
$$

By using the properties of those coordinates and the change of variable formula (8), we easily find that, for some $C>0$ depending only on the geometry of $\Omega$, we have

$$
\left\{\begin{array}{l}
\operatorname{diam}\left(K_{\xi, \sigma}\right) \leq C(\Delta x+\xi) \\
\frac{1}{C}|\sigma| \xi \leq\left|K_{\xi, \sigma}\right| \leq C|\sigma| \xi
\end{array}\right.
$$




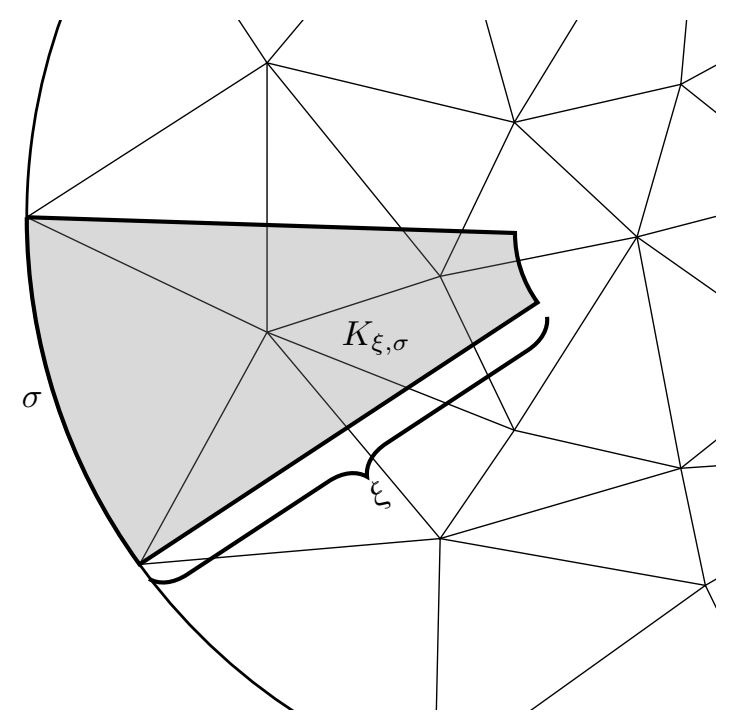

Figure 5: Illustration of the definition of $K_{\xi, \sigma}$ for some exterior face $\sigma \in \partial \mathcal{T}^{\text {ext }}$

We set $K_{\xi, \sigma}^{n}=\left(t^{n}, t^{n+1}\right) \times K_{\xi, \sigma}$ and we observe that, thanks to the Lipschitz-continuity in space of $f$ we have, for any $(t, y) \in \sigma^{n}$

$$
\left|f(t, y)-f_{K_{\xi, \sigma}} f(t, x) d x\right| \leq L_{f} \operatorname{diam}\left(K_{\xi, \sigma}\right) \leq C L_{f}(\Delta x+\xi)
$$

By writing $f=\left(f-f_{K_{\xi, \sigma}} f(t, x) d x\right)+f_{K_{\xi, \sigma}} f(t, x) d x$ in the definition of $\mathcal{T}_{n, \sigma}$, it follows that

$$
\begin{aligned}
\mathcal{T}_{n, \sigma} & \leq 2 C L_{f}(\Delta x+\xi) \Delta t|\sigma|+|\sigma| \int_{t^{n}}^{t^{n+1}}\left|f_{K_{\xi, \sigma}} f(t, x) d x-f_{K_{\xi, \sigma}^{n}} f\right| d t \\
& \leq 2 C L_{f}(\Delta x+\xi) \Delta t|\sigma|+|\sigma| f_{K_{\xi, \sigma}} \int_{t^{n}}^{t^{n+1}}\left(\left|f(t, x)-f_{t^{n}}^{t^{n+1}} f(s, x) d s\right| d t\right) d x \\
& \leq 2 C L_{f}(\Delta x+\xi) \Delta t|\sigma|+\Delta t \frac{|\sigma|}{\left|K_{\xi, \sigma}\right|} \int_{K_{\xi, \sigma}} \int_{t^{n}}^{t^{n+1}}\left|\partial_{t} f(t, x)\right| d t d x .
\end{aligned}
$$

Finally, with the properties (39), we get

$$
\mathcal{T}_{n, \sigma} \leq 2 C L_{f}(\Delta x+\xi) \Delta t|\sigma|+C \frac{\Delta t}{\xi} \int_{K_{\xi, \sigma}} \int_{t^{n}}^{t^{n+1}}\left|\partial_{t} f(t, x)\right| d t d x
$$

and by summation over $n$ and $\sigma$, the first claim follows.

For proving (38) we simply bound the integral of $\left|\partial_{t} f\right|$ over $\Omega_{\xi}$ by the same integral on the whole domain $\Omega$, and then we choose $\xi=\sqrt{\Delta t}$, with $\Delta t$ small enough so that the condition $\xi<\xi_{\Omega}$ is fulfilled.

2. Let $K \in \mathcal{T}$ be any cell in the mesh and $\sigma=K \mid L$ any face of $K$. We can make the same computation as before but replacing the set $K_{\xi, \sigma}$ by the actual cell $K$. We obtain

$$
\mathcal{T}_{n, K \mid L} \leq 2 C L_{f} \operatorname{diam}(K) \Delta t|K| L\left|+\Delta t \frac{|K| L \mid}{|K|} \iint_{K^{n}}\right| \partial_{t} f(t, x) \mid d t d x .
$$


Using (17) and 19, we deduce that

$$
\begin{aligned}
\sum_{\substack{0 \leq n<N \\
K \mid L \in \partial \mathcal{T}}} \mathcal{T}_{n, K \mid L} & \leq \frac{2 C L_{f}}{\alpha} \sum_{\substack{0 \leq n<N \\
K \in \mathcal{T}}} \Delta t|K|+\frac{1}{\alpha^{2}} \sum_{\substack{0 \leq n<N \\
K \in \mathcal{T}}} \iint_{K^{n}}\left|\partial_{t} f\right| d x d t \\
& \leq \frac{2 C L_{f} T|\Omega|}{\alpha}+\frac{1}{\alpha^{2}} \iint_{\Omega^{T}}\left|\partial_{t} f\right| d x d t .
\end{aligned}
$$

We may now go back to the proposition.

Proof of Proposition 4.7. To prove the first estimate, we assume that $u^{0}$ is smooth. We write

$$
\int_{\Omega}\left|u^{0}-u_{\Delta x}^{0}\right| d x=\sum_{K \in \mathcal{T}^{\text {ext }}} \int_{K}\left|u^{0}-u_{K}^{0}\right| d x+\sum_{K \in \mathcal{T}^{\text {int }}} \int_{K}\left|u-u_{K}^{0}\right| d x .
$$

For interior cells (that are convex), we use the definition of $u_{\Delta x}^{0}$ in $[20$, Lemma 3.2 and 15 whereas for exterior cells we simply use $L^{\infty}$-bounds to get

$$
\begin{aligned}
\int_{\Omega}\left|u^{0}-u_{\Delta x}^{0}\right| d x & \leq \frac{C}{\alpha^{d}} \sum_{K \in \mathcal{T}^{\text {ext }}} \operatorname{diam}(K) \int_{K}\left|\nabla u^{0}\right| d x+2\left\|u^{0}\right\|_{L^{\infty}(\Omega)} \sum_{K \in \mathcal{T}^{\text {ext }}}|K| \\
& \leq C_{\alpha} \Delta x \int_{\Omega}\left|\nabla u^{0}\right| d x+C_{\Omega}\left\|u^{0}\right\|_{L^{\infty}(\Omega)} \Delta x .
\end{aligned}
$$

The claim follows by the density property 6 .

Let us consider now the second estimate.

- We first prove that

$$
\iint_{\Gamma^{T}}\left|u_{b}-u_{b, \Delta x}\right| d \sigma d t \leq C\left(\left\|u_{b}\right\|_{L^{\infty}\left(\Gamma^{T}\right)}+\operatorname{TV}_{\Gamma^{T}}\left(u_{b}\right)\right) \sqrt{\Delta x} .
$$

By a standard convolution process similar to the one proposed in 32 but on $\Gamma^{T}$, we may build a smooth function $u_{b}^{\epsilon}: \Gamma^{T} \rightarrow \mathbb{R}$ such that

$$
\left\|\nabla_{t, \tilde{x}} u_{b}^{\epsilon}\right\|_{L^{\infty}\left(\Gamma^{T}\right)} \leq \frac{C}{\epsilon}\left\|u_{b}\right\|_{L^{\infty}\left(\Gamma^{T}\right)}, \quad\left\|u_{b}-u_{b}^{\epsilon}\right\|_{L^{1}\left(\Gamma^{T}\right)} \leq C \epsilon \operatorname{TV}_{\Gamma^{T}}\left(u_{b}\right) .
$$

Since $u_{b}^{\epsilon}$ is Lipschitz continuous we immediately see that, by definition of $u_{b, \Delta x}^{\epsilon}$ (see 20 ), we have

$$
\left\|u_{b}^{\epsilon}-u_{b, \Delta x}^{\epsilon}\right\|_{L^{1}\left(\Gamma^{T}\right)} \leq C\left\|\nabla_{t, \tilde{x}} u_{b}^{\epsilon}\right\|_{L^{\infty}\left(\Gamma^{T}\right)}(\Delta t+\Delta x) \leq \frac{C}{\epsilon}\left\|u_{b}\right\|_{L^{\infty}\left(\Gamma^{T}\right)}(\Delta t+\Delta x) .
$$

Moreover, we have

$$
\left\|u_{b}-u_{b}^{\epsilon}-\left(u_{b, \Delta x}-u_{b, \Delta x}^{\epsilon}\right)\right\|_{L^{1}\left(\Gamma^{T}\right)} \leq 2\left\|u_{b}-u_{b}^{\epsilon}\right\|_{L^{1}\left(\Gamma^{T}\right)} \leq C \epsilon \operatorname{TV}_{\Gamma^{T}}\left(u_{b}\right) .
$$

Combining the two inequalities, and using the triangle inequality, we find

$$
\left\|u_{b}-u_{b, \Delta x}\right\|_{L^{1}\left(\Gamma^{T}\right)} \leq \frac{C}{\epsilon}\left\|u_{b}\right\|_{L^{\infty}\left(\Gamma^{T}\right)}(\Delta t+\Delta x)+C \epsilon \operatorname{TV}_{\Gamma^{T}}\left(u_{b}\right)
$$

and the claim follows by 19 and by choosing $\epsilon=\sqrt{\Delta x}$. Note that, with additional regularity assumptions on the mesh, one can prove a stronger estimate in $\Delta x$ instead of $\sqrt{\Delta x}$ but this is useless for our purpose. 
- We define $v=\mathbf{v} \cdot \mathbf{n}$, where $\mathbf{n}$ is the smooth extension of the unit outward normal field introduced in 1.3 . This function $v$ satisfies the same regularity assumptions as $\mathbf{v}$. We denote by $v_{\Delta x}$ the piecewise constant function

$$
v_{\Delta x}(t, x)=\int_{K^{n} \mid L^{n}} v=V_{K L}^{n} \text { if } \forall t \in\left(t^{n}, t^{n+1}\right), \forall x \in K \mid L \in \partial \mathcal{T},
$$

and we set

$$
\Gamma_{\text {in,app }}^{T}:=\left\{(t, x) \in \Gamma^{T}, \quad v_{\Delta x}(t, x)<0\right\}=\bigcup_{\substack{n<N \\ \sigma^{n} \in \partial \mathcal{T}_{-}^{n}}} \sigma^{n} .
$$

The difficulties come from the fact we have neither $\Gamma_{\mathrm{in}, \text { app }}^{T} \subset \Gamma_{\mathrm{in}}^{T}$ nor $\Gamma_{\mathrm{in}}^{T} \subset \Gamma_{\mathrm{in}, \mathrm{app}}^{T}$.

However, we observe that $|\mathbf{v} \cdot \mathbf{n}|=-(\mathbf{v} \cdot \mathbf{n})$ on $\Gamma_{\text {in }}^{T}$, so that we can write

$$
\begin{aligned}
\iint_{\Gamma_{\mathrm{in}}^{T}}\left|u_{b}-\gamma u_{\Delta x}\right||\mathbf{v} \cdot \mathbf{n}| d \sigma d t= & -\iint_{\Gamma_{\mathrm{in}}^{T}}\left|u_{b}-\gamma u_{\Delta x}\right|\left(\mathbf{v} \cdot \mathbf{n}-v_{\Delta x}\right) d \sigma d t \\
& -\iint_{\Gamma_{\mathrm{in}}^{T}}\left|u_{b}-\gamma u_{\Delta x}\right| v_{\Delta x} d \sigma d t .
\end{aligned}
$$

In the first term we simply bound $u_{b}$ and $\gamma u_{\Delta x}$ by $\|u\|_{L^{\infty}\left(\Omega^{T}\right)}$. For the second integral, we use that $v_{\Delta x} \geq 0$ on $\Gamma_{\text {in }}^{T} \backslash \Gamma_{\text {in,app }}^{T}$ to write

$$
\begin{aligned}
-\iint_{\Gamma_{\text {in }}^{T}}\left|u_{b}-\gamma u_{\Delta x}\right| v_{\Delta x} d \sigma d t & \leq-\iint_{\Gamma_{\text {in }}^{T} \cap \Gamma_{\text {in,app }}^{T}}\left|u_{b}-\gamma u_{\Delta x}\right| v_{\Delta x} d \sigma d t \leq\|\mathbf{v}\|_{\infty} \iint_{\Gamma_{\text {in app }}^{T},}\left|u_{b}-\gamma u_{\Delta x}\right| d \sigma d t \\
& =\|\mathbf{v}\|_{\infty} \iint_{\Gamma_{\text {in,app }}^{T}}\left|u_{b}-u_{b, \Delta x}\right| d \sigma d t \\
& \leq\|\mathbf{v}\|_{\infty} \iint_{\Gamma^{T}}\left|u_{b}-u_{b, \Delta x}\right| d \sigma d t .
\end{aligned}
$$

We have used here the fact that, by definition of the scheme 22 , the trace of the approximate solution $\gamma u_{\Delta x}$ on the set $\Gamma_{\mathrm{in}, \text { app }}^{T}$ is given by $u_{b, \Delta x}$. We have thus proved

$$
\iint_{\Gamma_{\text {in }}^{T}}\left|u_{b}-\gamma u_{\Delta x}\right||\mathbf{v} \cdot \mathbf{n}| d \sigma d t \leq 2\|u\|_{L^{\infty}\left(\Omega^{T}\right)} \iint_{\Gamma^{T}}\left|v-v_{\Delta x}\right| d \sigma d t+\|\mathbf{v}\|_{\infty} \iint_{\Gamma^{T}}\left|u_{b}-u_{b, \Delta x}\right| d \sigma d t .
$$

Using the first point of Lemma 6.1 for the function $v$, the CFL condition 23 and the previous estimate 40, we finally obtain

$$
\iint_{\Gamma_{\text {in }}^{T}}\left|u_{b}-\gamma u_{\Delta x}\right||\mathbf{v} \cdot \mathbf{n}| d \sigma d t \leq C\left(\|u\|_{L^{\infty}\left(\Omega^{T}\right)}+\operatorname{TV}_{\Gamma^{T}}\left(u_{b}\right)\right) \sqrt{\Delta x} .
$$

\subsection{Results concerning the approximate weak formulation}

Proof of Proposition 4.8. The Cauchy-Schwarz theorem yields

$$
\begin{aligned}
\left|\mu_{\Delta x}\left(u_{\epsilon}\right)\right| & =\left|\sum_{\substack{0 \leq n<N \\
K \in \mathcal{T}}}\right| K\left|\left(u_{K}^{n+1}-u_{K}^{n}\right)\left(f_{K^{n}} u_{\epsilon}(s, x)-u_{\epsilon}\left(t^{n+1}, x\right) d x d s\right)\right| \\
& \leq \sqrt{\mathcal{D}_{\Delta x}\left(u_{\Delta x}, T\right)}\left(\sum_{\substack{0 \leq n<N \\
K \in \mathcal{T}}}|K|\left(f_{K^{n}}\left(u_{\epsilon}(s, x)-u_{\epsilon}\left(t^{n+1}, x\right)\right) d s d x\right)^{2}\right)^{1 / 2} .
\end{aligned}
$$


Similarly, the term $\nu_{\Delta x}\left(u_{\epsilon}\right)$ term can be rewritten as

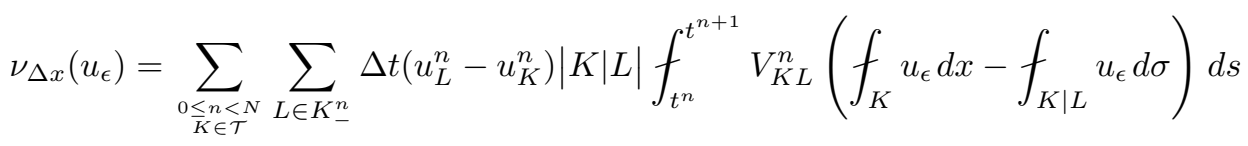

$$
\begin{aligned}
& +\sum_{\substack{0 \leq n<N \\
K \in \mathcal{T}}} \sum_{L \in K_{-}^{n}} \Delta t\left(u_{L}^{n}-u_{K}^{n}\right)|K| L \mid f \int_{K^{n} \mid L^{n}} u_{\epsilon}\left(V_{K L}^{n}-\mathbf{v} \cdot \mathbf{n}_{K L}\right) d \sigma d s
\end{aligned}
$$

and thus

$$
\begin{aligned}
\left|\nu_{\Delta x}\left(u_{\epsilon}\right)\right| \leq & \sqrt{\mathcal{D}_{\Delta x}\left(u_{\Delta x}, T\right)}\left(\sum_{\substack{0 \leq n<N \\
K \in \mathcal{T}}} \sum_{L \in K_{-}^{n}} \Delta t f_{t^{n}}^{t^{n+1}}|K| L|| V_{K L}^{n} \mid\left(f_{K} u_{\epsilon} d x-f_{K \mid L} u_{\epsilon} d \sigma\right)^{2}\right)^{1 / 2} \\
& +2\|u\|_{\infty} \sum_{\substack{0 \leq n<N \\
K \in \mathcal{T}}} \sum_{L \in K_{-}^{n}} \Delta t|K| L\left|f f_{K^{n} \mid L^{n}}\right| u_{\epsilon}|| V_{K L}^{n}-\mathbf{v} \cdot \mathbf{n}_{K L} \mid d \sigma d s
\end{aligned}
$$

The claim is thus a consequence of the following two Lemmas 6.3 and 6.4 .

Lemma 6.3. Under the same assumptions as in Theorem 4.1, there exists a $C>0$ depending only on $\Omega$, $T$, $\mathbf{v}$ and $\alpha$ such that

$$
\left|\sum_{\substack{0 \leq n<N \\ K \in \mathcal{T}}} \sum_{L \in K_{-}^{n}} \Delta t\right| K|L| f_{K^{n} \mid L^{n}} u_{\epsilon}\left(V_{K L}^{n}-\mathbf{v} \cdot \mathbf{n}_{K L}\right) d \sigma d s \mid \leq C \frac{\Delta x}{\epsilon}\|u\|_{L^{\infty}\left(\Omega^{T}\right)} .
$$

Proof. By definition of $V_{K L}^{n}$, the function $V_{K L}^{n}-\left(\mathbf{v} \cdot \mathbf{n}_{K L}\right)$ has a zero average on $K^{n} \mid L^{n}$, therefore we can replace $u_{\epsilon}$ by $u_{\epsilon}-\left(f_{K^{n} \mid L^{n}} u_{\epsilon}\right)$ in each term, and since $u_{\epsilon}$ is Lipschitz continuous, this quantity is bounded by $(\Delta t+\Delta x)\left\|\nabla_{t, x} u_{\epsilon}\right\|_{L^{\infty}\left(\Omega^{T}\right)}$. It follows that

$$
\begin{aligned}
& \left|\sum_{\substack{0 \leq n<N \\
K \in \mathcal{T}}} \sum_{L \in K_{-}^{n}} \Delta t\right| K|L| f_{K^{n} \mid L^{n}} u_{\epsilon}\left(V_{K L}^{n}-\mathbf{v} \cdot \mathbf{n}_{K L}\right) d \sigma d s \mid \\
& \quad \leq(\Delta t+\Delta x)\left\|\nabla_{t, x} u_{\epsilon}\right\|_{L^{\infty}\left(\Omega^{T}\right)}\left(\sum_{\substack{0 \leq n<N \\
K \backslash L \in \partial \mathcal{T}}} \Delta t|K| L\left|\|_{K^{n} \mid L^{n}}\right| V_{K L}^{n}-\mathbf{v} \cdot \mathbf{n}_{K L} \mid d \sigma d s\right) \leq C \frac{\Delta x}{\epsilon}\|u\|_{L^{\infty}\left(\Omega^{T}\right)},
\end{aligned}
$$

where we have used 19, Proposition 4.4 and the second point of Lemma 6.1 .

Lemma 6.4. Under the same assumptions as in Theorem 4.1, there exists a $C>0$ depending only on $\Omega, T$, $\mathbf{v}$ and $\alpha$ such that

$$
\begin{aligned}
\sum_{\substack{0 \leq n<N \\
K \in \mathcal{T}}} \sum_{L \in K_{-}^{n}} \Delta t f_{t^{n}}^{t^{n+1}}|K| L \mid V_{K L}^{n}\left(f_{K} u_{\epsilon} d x-f_{K \mid L} u_{\epsilon} d \sigma\right)^{2} & \leq C\|u\|_{L^{1}(0, T ; \mathrm{BV}(\Omega))}\|u\|_{\infty} \frac{\Delta x}{\epsilon}+C\|u\|_{L^{\infty}\left(\Omega^{T}\right)}^{2} \frac{\Delta x^{2}}{\epsilon^{2}}
\end{aligned}
$$

and

$$
\sum_{\substack{0 \leq n<N \\ K \in \mathcal{T}}}|K|\left(f_{K^{n}}\left(u_{\epsilon}(t, x)-u_{\epsilon}\left(t^{n+1}, x\right)\right) d x d t\right)^{2} \leq C\|u\|_{L^{1}(0, T ; \mathrm{BV}(\Omega))}\|u\|_{\infty} \frac{\Delta t}{\epsilon}
$$


Proof. Let us denote by $T_{\text {int }}$ (resp. $T_{\text {ext }}$ ) the sum of the contributions of interior (resp. exterior) cells in the left-hand side of 42 .

Since the interior cells are polyhedral, convex and satisfy 15 , we can use Lemma 3.1 as follows

$$
\begin{aligned}
& T_{\mathrm{int}}:=\sum_{\substack{0 \leq n<N \\
K \in \mathcal{T}^{\text {int }}}} \sum_{L \in K_{-}^{n}} \Delta t f_{t^{n}}^{t^{n+1}}|K| L \mid V_{K L}^{n}\left(f_{K} u_{\epsilon} d x-f_{K \mid L} u_{\epsilon} d \sigma\right)^{2} \\
& \leq\left. C \sum_{\substack{0 \leq n<N \\
K \in \mathcal{T}^{\text {int }}}} \sum_{L \in K_{-}^{n}} \Delta t f_{t^{n}}^{t^{n+1}}|K| L\left|V_{K L}^{n} \frac{\operatorname{diam}(K)}{|K| L \mid} \int_{K}\right| \nabla_{x} u_{\epsilon}\right|^{2} d x \\
& \leq C\|\mathbf{v}\|_{\infty} \Delta x \sum_{\substack{0 \leq n<N \\
K \in \mathcal{T}^{\mathrm{int}}}} \sum_{L \in K_{-}^{n}} \iint_{K^{n}}\left|\nabla_{x} u_{\epsilon}\right|^{2} d x d t
\end{aligned}
$$

By using (16), and Proposition 4.4, we get

$$
T_{\mathrm{int}} \leq \frac{C}{\alpha} \Delta x \int_{0}^{T} \int_{\Omega}\left|\nabla_{x} u_{\epsilon}\right|^{2} d x d t \leq C \frac{\Delta x}{\alpha}\left\|\nabla_{x} u_{\epsilon}\right\|_{L^{1}\left(\Omega^{T}\right)}\left\|\nabla_{x} u_{\epsilon}\right\|_{L^{\infty}\left(\Omega^{T}\right)} \leq C \frac{\Delta x}{\epsilon}\|u\|_{L^{\infty}\left(\Omega^{T}\right)}\|u\|_{L^{1}(0, T ; \mathrm{BV}(\Omega))} .
$$

We can now estimate as follows the contribution of the exterior cells (that may be curved and non convex) without using Lemma 3.1

$$
\begin{aligned}
T_{\text {ext }} & :=\sum_{\substack{0 \leq n<N \\
K \in \mathcal{T}_{\text {ext }}}} \sum_{L \in K_{-}^{n}} \Delta t f_{t^{n}}^{t^{n+1}}|K| L \mid V_{K L}^{n}\left(f_{K} u_{\epsilon} d x-f_{K \mid L} u_{\epsilon} d \sigma\right)^{2} \\
& \leq C\|\mathbf{v}\|_{L^{\infty}\left(\Omega^{T}\right)} \sum_{\substack{0 \leq n<N \\
K \in \mathcal{T}^{\text {ext }}}} \sum_{L \in K_{-}^{n}} \Delta t|K| L \mid(\operatorname{diam}(K))^{2}\left\|\nabla_{x} u_{\epsilon}\right\|_{L^{\infty}\left(\Omega^{T}\right)}^{2}
\end{aligned}
$$

By using 17), we deduce

$$
\begin{aligned}
T_{\text {ext }} & \leq \frac{C \Delta x}{\alpha}\left\|\nabla_{x} u_{\epsilon}\right\|_{L^{\infty}\left(\Omega^{T}\right)}^{2} \sum_{\substack{0 \leq n<N \\
K \in \mathcal{T}^{\mathrm{ext}}}} \Delta t|K| \\
& \leq \frac{C T \Delta x}{\alpha}\left\|\nabla_{x} u_{\epsilon}\right\|_{L^{\infty}\left(\Omega^{T}\right)}^{2}\left|\bigcup_{K \in \mathcal{T}^{\mathrm{ext}}} K\right| .
\end{aligned}
$$

By definition of the exterior cells, we have $\bigcup_{K \in \mathcal{T} \text { ext }} K \subset \mathcal{O}_{\Delta x}$, and thus, with Proposition 4.4 we get

$$
T_{\text {ext }} \leq \frac{C T|\Gamma| \Delta x^{2}}{\alpha \epsilon^{2}}\|u\|_{L^{\infty}\left(\Omega^{T}\right)}^{2}
$$

To prove the estimate (43, we simply use the Jensen inequality, the inequality (18) and finally Proposition 4.4 as follows

$$
\begin{aligned}
\sum_{\substack{0 \leq n<N \\
K \in \mathcal{T}}}|K|\left(\int_{K^{n}}\left(u_{\epsilon}(s, x)-u_{\epsilon}\left(t^{n+1}, x\right)\right) d s d x\right)^{2} \\
\quad \leq \sum_{\substack{0 \leq n<N \\
K \in \mathcal{T}}} \int_{K}\left|f_{t^{n}}^{t^{n+1}}\left(u_{\epsilon}(t, x)-u_{\epsilon}\left(t^{n+1}, x\right)\right) d t\right|^{2} d x \\
\leq \Delta t \sum_{\substack{0 \leq n<N \\
K \in \mathcal{T}}} \iint_{K^{n}}\left|\partial_{t} u_{\epsilon}(t, x)\right|^{2} d x d t \\
\leq \Delta t\left\|\partial_{t} u_{\epsilon}\right\|_{L^{\infty}\left(\Omega^{T}\right)}\left\|\partial_{t} u_{\epsilon}\right\|_{L^{1}\left(\Omega^{T}\right)} \\
\leq C \frac{\Delta t}{\epsilon}\|u\|_{L^{\infty}\left(\Omega^{T}\right)}\|u\|_{L^{1}(0, T ; \mathrm{BV}(\Omega))}
\end{aligned}
$$




\section{BV regularity of solutions to transport problems}

The aim of this final section is to prove the BV regularity of the exact solution of the linear transport problem (1), that is to prove Theorem 2.5.

The main reference concerning the existence of a solution with bounded total variation to a conservation law on a bounded domain is the very famous paper by Bardos, Leroux and Nédélec [BlRN79]. Their approach have recently been revisited in [CR15], making the case of nonhomogenous boundary conditions more rigorous. Those two papers deal with a framework that is far more general than the linear advection equation we consider here. However, the regularity assumption on the boundary data which is required in both cases is very strong (with our notation it would amount to require that, at least, $u_{b} \in \mathcal{C}^{3}([0, T] \times \Gamma)$ ). Therefore, it cannot directly be applied to the case where $u_{b}$ is a BV function as needed in our proof of the error estimate (we recall that we used this theorem with characteristic functions of finite perimeter sets as initial and boundary data).

For this reason, we present here a proof of the BV regularity of the solution of the linear transport equation assuming only that the initial and boundary data are themselves bounded and with bounded variation, that is (2) and (3). Actually, we will prove a slightly stronger result since the only assumptions we shall need for $\mathbf{v}$ are the following

$$
\mathbf{v} \in L^{\infty}\left(\Omega^{T}\right) \text {, and } L_{\mathbf{v}}:=\left\|\nabla_{x} \mathbf{v}\right\|_{L^{\infty}\left(\Omega^{T}\right)}<+\infty .
$$

In particular we shall not assume that $\mathbf{v}$ is divergence free.

Remark 7.1. We would like to mention again that the previous result may fail if we only assume that $u_{b}$ has only bounded variation on the inflow part $\Gamma_{\text {in }}^{T}$ of the boundary and not on the whole boundary $\Gamma^{T}$, see the counter-example given in Remark 2.7 .

\subsection{Sketch of proof}

The proof is basically split into two parts. In the first part, we prove the result in the case where $\Omega$ is a half-space and $u^{0}, u_{b}$ are compactly supported. The strategy is very close in spirit to the one in [CH99] with a treatment of the boundary similar to OV06] (but less technical since we use a Cartesian grid). For this reason we may omit some straightforward computations and details. In the second part we recover the complete result by usual localization arguments and change of variables that are detailed in Section 7.4.

From now on, and until we reach Section 7.4 , we assume that $\Omega=\left\{\left(x_{1}, \ldots, x_{d}\right) \in \mathbb{R}^{d}: x_{d}>0\right\}$, so that $\Gamma=\mathbb{R}^{d-1} \times\{0\}$ and we choose a final time $T>0$. In order to prove the BV regularity of the exact solution in this setting, we shall prove a discrete BV bound for the approximate solution given by the upwind scheme (21) on a uniform Cartesian grid of $\Omega$. Using such a grid is crucial for it is well known that the uniform discrete $\overline{\mathrm{BV}}$ bound does not hold on a general grid for such approximations (see the counterexample given by Després Des04a).

However, on a uniform Cartesian grid, we shall obtain the uniform discrete BV estimate and the BV estimate for the exact solution will follow since the approximate solution given by the upwind scheme is known to converge in $\mathcal{C}^{0}\left([0, T], L^{1}(\Omega)\right)$ towards the exact solution of the transport problem (see Boy12 for instance).

\subsection{Notations}

For a given $N \geq 1$, we set $\Delta t=T / N$ and then we decide to choose

$$
\Delta x:=2 d\|\mathbf{v}\|_{\infty} \Delta t
$$

so that the CFL condition will be automatically satisfied. Since we use a uniform Cartesian grid, we can use adapted notation based on a multi-indices. We refer to Figure 6 .

- The cells in $\mathcal{T}$ are given by

$$
K:=\left(k_{1} \Delta x,\left(k_{1}+1\right) \Delta x\right) \times \cdots \times\left(k_{d} \Delta x,\left(k_{d}+1\right) \Delta x\right), \text { with }\left(k_{1}, \ldots, k_{d}\right) \in \mathbb{Z}^{d-1} \times \mathbb{N} .
$$

- We identify each cell $K$ with the corresponding multi-index $\left(k_{1}, \ldots, k_{d}\right)$ in such a way that the neighboring cells of $K$ can be described by

$$
K+e_{i}, i \in\{-d, \ldots, d\} \backslash\{0\}
$$




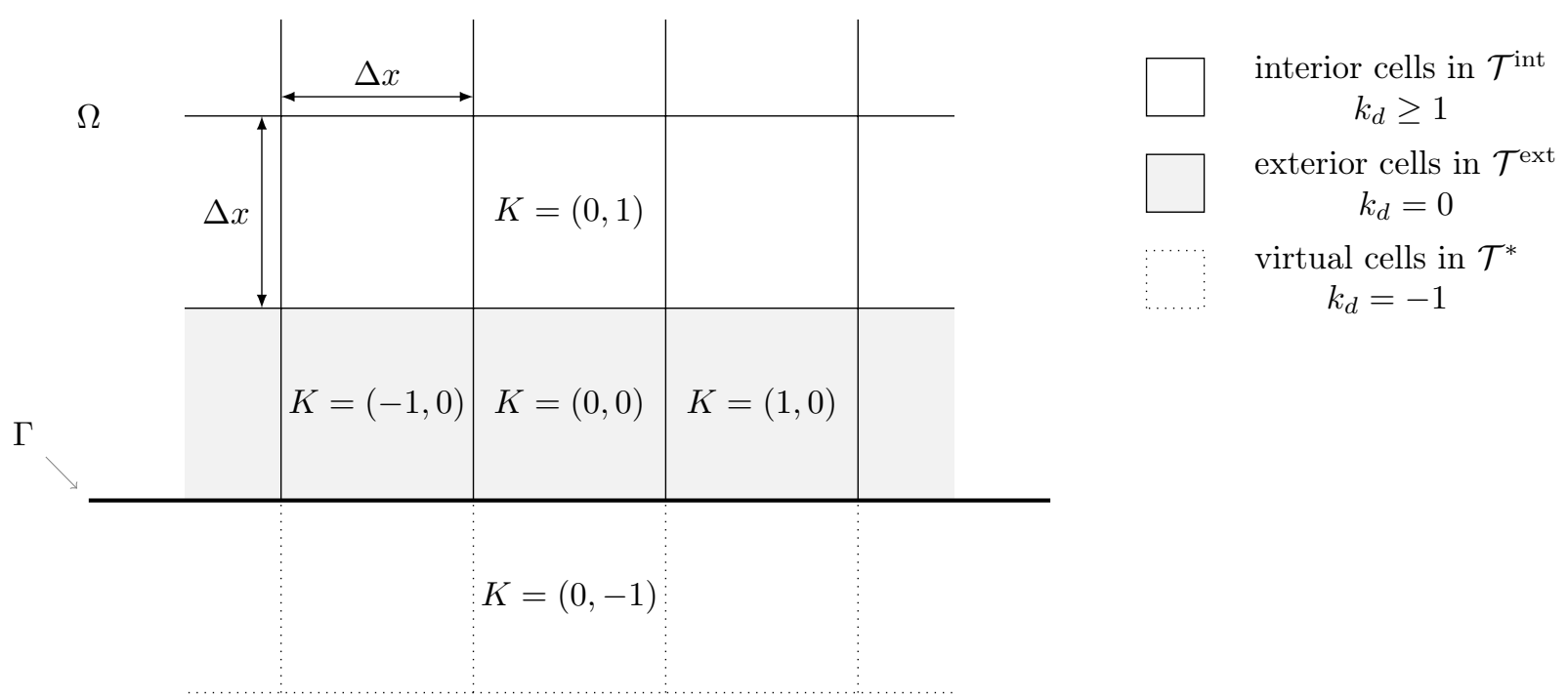

Figure 6: Notations for the Cartesian mesh

where $e_{1}, \ldots, e_{d}$ are the vectors of the canonical basis of $\mathbb{R}^{d}$, and $e_{-i}=-e_{i}$. Conventionally we set $e_{0}=0$, so that $K+e_{i}=K$ for $i=0$.

- The interior and exterior meshes are given by

$$
\mathcal{T}^{\text {int }}=\left\{K=\left(k_{1}, \ldots, k_{d}\right), \text { such that } k_{d} \geq 1\right\}, \quad \mathcal{T}^{\text {ext }}=\left\{K=\left(k_{1}, \ldots, k_{d}\right), \text { such that } k_{d}=0\right\},
$$

and the virtual cells needed to deal with the boundary data are simply defined, by extension, as follows

$$
\mathcal{T}^{*}:=\left\{K=\left(k_{1}, \ldots, k_{d}\right), \text { such that } k_{d}=-1\right\},
$$

Even if $\mathbf{v}$ is not divergence free, we can use the same upwind scheme $21-22$ to compute a convergent approximation of the transport problem (see Boy12]).

Using the particular geometry of the mesh, we can rewrite the scheme under the more compact form

$$
u_{K}^{n+1}=u_{K}^{n}+\sum_{-d \leq i \leq d} a_{K, K+e_{i}}^{n}\left(u_{K+e_{i}}^{n}-u_{K}^{n}\right),
$$

where the coefficient $a_{K, K+e_{i}}^{n}$ is defined as follows: $a_{K, K}^{n}=0$ and for $i \in\{-d, \ldots, d\} \backslash\{0\}, n<N$, we set

$$
a_{K, K+e_{i}}^{n}:= \begin{cases}-\frac{\Delta t}{\Delta x} V_{K, K+e_{i}}^{n} & \text { if } V_{K, K+e_{i}}<0 \\ 0 & \text { otherwise. }\end{cases}
$$

We recall that

$$
V_{K, K+e_{i}}^{n}:=\frac{1}{\Delta x^{d-1} \Delta t} \int_{n \Delta t}^{(n+1) \Delta t} \int_{K \mid K+e_{i}} \mathbf{v}(t, x) \cdot \mathbf{n}_{K, K+e_{i}} d \sigma(x) d t,
$$

where $K \mid K+e_{i}$ is the common face to the cells $K$ and $K+e_{i}$, and $\mathbf{n}_{K, K+e_{i}}=e_{i}$ is the normal vector to this face, oriented outward of $K$. We also set $V_{K, K}^{n}=0$ and

$$
u_{K}^{n}=u_{b, K \mid K+e_{d}}^{n}:=\frac{1}{\Delta x^{d-1} \Delta t} \int_{n \Delta t}^{(n+1) \Delta t} \int_{L \mid L+e_{d}} u_{b}(t, x) d \sigma(x) d t, \quad \forall K \in \mathcal{T}^{*}, \forall n<N .
$$


Observe that the value of $u_{K}^{n}=u_{b, K \mid K+e_{d}}^{n}$ with $K \in \mathcal{T}^{*}$ only appears in the scheme if $V_{K+e_{d}, K}<0$ (or equivalently if $\left.a_{K+e_{d}, K}>0\right)$. However, all the values of $u_{b, K \mid K+e_{d}}^{n}$ will come up in the BV estimate, in particular in the terms introduced in Definition 7.2

To simplify some computations, we shall define values of the approximate solution outside the extended mesh $\mathcal{T} \cup \mathcal{T}^{*}$ as follows

$$
u_{K+e_{-d}}^{n}:=u_{K}^{n}, \quad \forall K \in \mathcal{T}^{*}, \forall n<N .
$$

This convention simply allows to deal, without particular care, with quantities like $u_{K+e_{j}}^{n}-u_{K}^{n}$ for any $j \in$ $\{-d, \ldots, d\}$ and any $K \in \mathcal{T} \cup \mathcal{T}^{*}$, even for $K \in \mathcal{T}^{*}$ and $j=-d$.

Our aim is to evaluate the total variation of the numerical solution at any time $t^{n}$. To this end, we shall need special notations to deal with jumps of the solution or of its trace across faces.

Definition 7.2. • For any $n<N$ and any $K \in \mathcal{T}$, we define

$$
\left\{\begin{array}{l}
Q_{K}^{n}:=\sum_{-d \leq j \leq d}\left|u_{K}^{n}-u_{K+e_{j}}^{n}\right|, \\
B_{K}^{n}:=0 .
\end{array}\right.
$$

- For any $n<N$ and any $K \in \mathcal{T}^{*}$, we define

$$
\left\{\begin{array}{l}
Q_{K}^{n}:=\left|u_{K}^{n}-u_{K+e_{d}}^{n}\right|, \\
B_{K}^{n}:=\left|u_{K}^{n+1}-u_{K}^{n}\right|+\sum_{-d<j<d}\left|u_{K}^{n}-u_{K+e_{j}}^{n}\right| .
\end{array}\right.
$$

- For any $n<N$, we set

$$
\left\{\begin{aligned}
Q^{n} & :=\sum_{K \in \mathcal{T} \cup \mathcal{T}^{*}} Q_{K}^{n}, \\
B^{n} & :=\sum_{K \in \mathcal{T} \cup \mathcal{T}^{*}} B_{K}^{n}=\sum_{K \in \mathcal{T}^{*}} B_{K}^{n}
\end{aligned}\right.
$$

Observe that the indices $j= \pm d$ do not enter the definition of $B_{K}^{n}$.

Remark 7.3. The contribution of the virtual cells in $Q^{n}$ can also be written

$$
\sum_{K \in \mathcal{T}^{*}} Q_{K}^{n}=\sum_{K \in \mathcal{T}^{\mathrm{ext}}}\left|u_{K}^{n}-u_{K+e_{-d}}^{n}\right|
$$

All the terms $Q_{K}^{n}$ contain values produced by the numerical scheme and that need to be evaluated whereas all the terms in $B_{K}^{n}$ contain only values of the boundary data that are given, and in particular we have the following bound

Lemma 7.4. There exists $a C>0$ independent of $\Delta t$ and $\Delta x$ such that

$$
\Delta x^{d-1} \sum_{n<N-1} B^{n} \leq C \operatorname{TV}_{\Gamma^{T}}\left(u_{b}\right)
$$

Proof. Let $n<N-1$ and $K \in \mathcal{T}^{*}$. By using Lemma 1.1 to the function $u_{b}$, as well as the definition (47), we get

$$
B_{K}^{n} \leq \frac{1}{\Delta x^{d-1}} \operatorname{TV}_{\left(t^{n}, t^{n+2}\right) \times\left(K \mid K+e_{d}\right)}\left(u_{b}\right)+\frac{1}{\Delta x^{d-2} \Delta t} \sum_{-d<j<d} \operatorname{TV}_{\left(t^{n}, t^{n+1}\right) \times\left(\left(K \mid K+e_{d}\right) \cup\left(K+e_{j} \mid K+e_{j}+e_{d}\right)\right)}\left(u_{b}\right) .
$$

The claim follows by summation of those inequality and thanks to 45.

Remark 7.5. We shall use at different point the fact that

$$
\sum_{-d \leq j \leq d}\left|u_{K}^{n}-u_{K+e_{j}}^{n}\right| \leq B_{K}^{n}+Q_{K}^{n}, \quad \forall n<N, \forall K \in \mathcal{T} \cup \mathcal{T}^{*}
$$


Let us be more precise on the claim we are going to prove: we would like to find a bound on the total variation of $u_{\Delta x}\left(t^{N-1},.\right)$ and on the $L^{1}$ norm of the difference between the trace of the approximate solution and the discretization of the boundary data at time $t^{N-1}$. We thus define, for any $n$,

$$
\mathcal{V}^{n}=\mathrm{TV}_{\Omega}\left(u_{\Delta x}\left(t^{n}, .\right)\right)+\left\|\gamma u_{\Delta x}\left(t^{n}, .\right)-u_{b, \Delta x}\left(t^{n}, .\right)\right\|_{L^{1}(\Gamma)},
$$

and the goal is to bound $\mathcal{V}^{N-1}$. A simple computation shows that the first term is the sum of contributions of the jumps of the function across interior faces

$$
\operatorname{TV}_{\Omega}\left(u_{\Delta x}\left(t^{n}, .\right)\right)=\sum_{K \mid L \in \partial \mathcal{T}^{\text {int }}}|K| L|| u_{K}^{n}-u_{L}^{n} \mid,
$$

and that the second term is given by

$$
\left\|\gamma u_{\Delta x}\left(t^{n}, .\right)-u_{b, \Delta x}\left(t^{n}, .\right)\right\|_{L^{1}(\Gamma)}=\sum_{K \in \mathcal{T}^{*}}|K| K+e_{d}|| u_{K}^{n}-u_{K+e_{d}}^{n}\left|=\sum_{K \in \mathcal{T}^{*}}\right| K\left|K+e_{d}\right|\left|u_{K}^{n}-u_{b, K \mid K+e_{d}}^{n}\right| .
$$

It follows, by replacing sums over all the faces by sums over all the cells, that we have

$$
\mathcal{V}^{n}=\frac{\Delta x^{d-1}}{2} Q^{n}
$$

and thus we will now work on obtaining a bound on $Q^{n}$ for any $n$. The coefficient 2 above comes from the fact that, in the definition of $Q^{n}$, each face is counted twice.

We shall need to estimate the initial value $\mathcal{V}^{0}$ as follows.

Lemma 7.6. There exists a $C>0$, independent of $\Delta t$ and $\Delta x$, such that

$$
\mathcal{V}^{0} \leq \operatorname{TV}_{\Omega}\left(u^{0}\right)+\left\|\gamma_{\Gamma} u^{0}-u_{b}(0, .)\right\|_{L^{1}(\Gamma)}+C\left(\operatorname{TV}_{\mathcal{O}_{\Delta x}}\left(u^{0}\right)+\operatorname{TV}_{(0, \Delta t) \times \Gamma}\left(u_{b}\right)\right),
$$

where $u_{b}(0,$.$) is the usual notation for the trace \gamma_{\{0\} \times \Gamma} u_{b}$ as defined in the introduction.

Proof. The bound of the first term simply follows by summing the inequalities given by Lemma 1.1 applied to $f=u^{0}, R=h=\Delta x$ and to two neighboring cells $K$ and $K+e_{j}$.

Let us now study the second term in $T V^{0}$.

- By using Lemma 1.1 and the definition of the trace, we have

$$
\int_{\mathbb{R}^{d-1}}\left|\gamma_{\Gamma} u^{0}(y)-\frac{1}{\Delta x} \int_{0}^{\Delta x} u^{0}\left(y, x_{d}\right) d x_{d}\right| \leq \operatorname{TV}_{\mathcal{O}_{\Delta x}}\left(u^{0}\right)
$$

- Then for any exterior face $\sigma=\left[k_{1} \Delta x,\left(k_{1}+1\right) \Delta x\right) \times \cdots \times\left[k_{d-1} \Delta x,\left(k_{d-1}+1\right) \Delta x\right)$ and the associated exterior cell $K=\left[k_{1} \Delta x,\left(k_{1}+1\right) \Delta x\right) \times \cdots \times\left[k_{d-1} \Delta x,\left(k_{d-1}+1\right) \Delta x\right) \times[0, \Delta x) \in \mathcal{T}^{\text {ext }}$, we have

$$
\int_{\sigma}\left|\frac{1}{\Delta x} \int_{0}^{\Delta x} u^{0}\left(y, x_{d}\right) d x_{d}-f_{K} u^{0} d x\right| d y \leq \operatorname{TV}_{K}\left(u^{0}\right) .
$$

By summation over the faces in $\partial \mathcal{T}^{\text {ext }}$ and by using 51

$$
\left\|\gamma_{\Gamma} u^{0}-\gamma u_{\Delta x}^{0}\right\|_{L^{1}(\Gamma)} \leq 2 \operatorname{TV}_{\mathcal{O}_{\Delta x}}\left(u^{0}\right) .
$$

- Similarly, we can deal with the trace at time $t=0$ of $u_{b}$ and prove that

$$
\left\|u_{b}(0, .)-u_{b, \Delta x}(0, .)\right\|_{L^{1}(\Gamma)} \leq 2 \operatorname{TV}_{(0, \Delta t) \times \Gamma}\left(u_{b}\right) .
$$

Actually, a careful analysis of all the above computations, show that

$$
\mathcal{V}^{0} \underset{\Delta t \rightarrow 0}{\longrightarrow} \operatorname{TV}_{\Omega}\left(u^{0}\right)+\left\|\gamma_{\Gamma} u^{0}-u_{b}(0, .)\right\|_{L^{1}(\Gamma)}
$$




\subsection{Proof of the BV estimate in the Cartesian setting}

We can now move to the proof of our regularity result for a domain which is a half-space. More precisely, we shall prove the following result.

Proposition 7.7. There exists a $C>0$ that does not depend on $\Delta t, \Delta x$ such that

$$
\mathcal{V}^{n+1} \leq(1+C \Delta t) \mathcal{V}^{n}+C \Delta x^{d-1} B^{n}, \quad \forall n \in\{0, \ldots, N-2\} .
$$

From this result we easily deduce our BV estimate.

Theorem 7.8. Assume that $\Omega=\mathbb{R}^{d-1} \times(0,+\infty)$, that $u^{0}$ satisfies (2), $u_{b}$ satisfies (3) and $\mathbf{v}$ satisfies (44). Then, the unique solution $u$ of the transport problem (1) satisfies

$$
\sup _{t \in[0, T]} \operatorname{TV}_{\Omega}(u(t, .)) \leq C\left(\left\|\gamma_{\Gamma} u^{0}-u_{b}(0, .)\right\|_{L^{1}(\Gamma)}+\operatorname{TV}_{\Omega}\left(u^{0}\right)+\operatorname{TV}_{\Gamma^{T}}\left(u_{b}\right)\right)
$$

where $C>0$ depends only on $T$ and $\mathbf{v}$.

Proof. From (53) and the discrete Gronwall lemma we obtain

$$
\sup _{n<N} \mathcal{V}^{n} \leq C\left(\mathcal{V}^{0}+\sum_{n<N-1} \Delta x^{d-1} B^{n}\right)
$$

Using the bounds on the data given by Lemmas 7.4 and 7.6 we obtain

$$
\sup _{n<N} \mathcal{V}^{n} \leq C\left(\left\|\gamma_{\Gamma} u^{0}-u_{b}(0, .)\right\|_{L^{1}(\Gamma)}+\operatorname{TV}_{\Omega}\left(u^{0}\right)+\operatorname{TV}_{\Gamma^{T}}\left(u_{b}\right)\right)
$$

By the definition 50 of $\mathcal{V}^{n}$, and since $u_{\Delta x}$ is piecewise constant in time, we deduce that

$$
\sup _{t \in[0, T)} \operatorname{TV}_{\Omega}\left(u_{\Delta x}(t, .)\right) \leq C\left(\left\|\gamma_{\Gamma} u^{0}-u_{b}(0, .)\right\|_{L^{1}(\Gamma)}+\operatorname{TV}_{\Omega}\left(u^{0}\right)+\operatorname{TV}_{\Gamma^{T}}\left(u_{b}\right)\right)
$$

We use now the convergence result given in Boy12, which says that $u_{\Delta x} \underset{\Delta t \rightarrow 0}{\longrightarrow} u$ in $\mathcal{C}^{0}\left([0, T], L^{1}(\Omega)\right)$ to obtain the claim, by the semi-continuity property (5). Observe that the convergence result in Boy12 is only given on a bounded domain and for the implicit scheme, but it can be adapted immediately to the explicit scheme under the CFL condition. Moreover, when dealing with compactly supported data the proposed analysis is valid on an unbounded domain. This proves the claim.

It remains to establish Proposition 7.7 .

The first lemma below only depends on regularity property of the velocity field $\mathbf{v}$.

Lemma 7.9. For all $K \in \mathcal{T} \cup \mathcal{T}^{*}$, we have

$$
\left|a_{K, K+e_{i}}-a_{K+e_{j}, K+e_{j}+e_{i}}\right| \leq L_{\mathbf{v}} \Delta t, \quad \forall i, j \in\{-d, \ldots, d\}
$$

and

$$
\left|\sum_{-d \leq i \leq d} a_{K, K+e_{i}}^{n}-\sum_{-d \leq i \leq d} a_{K-e_{i}, K}^{n}\right| \leq\|\operatorname{div} \mathbf{v}\|_{L^{\infty}\left(\Omega^{T}\right)} \Delta t \leq C L_{\mathbf{v}} \Delta t .
$$

The proof is omitted since it simply uses the assumption on $\mathbf{v}$, the conservativity of the fluxes and the Stokes formula.

The main idea of the proof of Proposition 7.7 consists in using the definition of the scheme (46) to express the jump terms in $Q^{n+1}$ by the same kind of terms at time $t^{n}$. The main issue here is that the values $u_{K}^{n+1}$ for $K \in \mathcal{T}^{*}$ do not satisfy the scheme since they are explicitly defined by the boundary data by (47). 
We thus define, for $n<N$ and $K \in \mathcal{T} \cup \mathcal{T}^{*}$, the quantity

$$
R_{K}^{n}:= \begin{cases}0, & \text { if } K \in \mathcal{T}, \\ u_{K}^{n+1}-u_{K}^{n}-\sum_{-d \leq j \leq d} a_{K, K+e_{j}}^{n}\left(u_{K+e_{j}}^{n}-u_{K}^{n}\right), & \text { if } K \in \mathcal{T}^{*}\end{cases}
$$

in such a way that we have now the following equality for any $n<N$ and any $K \in \mathcal{T} \cup \mathcal{T}^{*}$

$$
u_{K}^{n+1}=u_{K}^{n}+\sum_{-d \leq j \leq d} a_{K, K+e_{j}}^{n}\left(u_{K+e_{j}}^{n}-u_{K}^{n}\right)+R_{K}^{n} .
$$

Thanks to the choice (45) and to the definition of the coefficients $a_{K, L}^{n}$ we immediately see that, under the CFL condition (23), we have the bound

$$
\left|R_{K}^{n}\right| \leq\left|B_{K}^{n}\right|+a_{K, K+e_{d}}\left|u_{K+e_{d}}^{n}-u_{K}^{n}\right|, \quad \forall K \in \mathcal{T}^{*}, \forall n .
$$

We may now find a suitable expression of the jump between the values on two neighboring cells at time $t^{n+1}$ as a function of similar quantities at time $t^{n}$ as follows.

Lemma 7.10. For all $K \in \mathcal{T}, n<N$ and $-d \leq j \leq d$, we have

$$
\begin{aligned}
u_{K}^{n+1}-u_{K+e_{j}}^{n+1}=( & \left.u_{K}^{n}-u_{K+e_{j}}^{n}\right)\left(1-\sum_{-d \leq i \leq d} a_{K, K+e_{i}}^{n}\right)+\sum_{-d \leq i \leq d} a_{K, K+e_{i}}^{n}\left(u_{K+e_{i}}^{n}-u_{K+e_{i}+e_{j}}^{n}\right) \\
& +\sum_{-d \leq i \leq d}\left(a_{K, K+e_{i}}^{n}-a_{K+e_{j}, K+e_{j}+e_{i}}^{n}\right)\left(u_{K+e_{j}+e_{i}}^{n}-u_{K+e_{j}}^{n}\right)+R_{K}^{n}-R_{K+e_{j}}^{n} .
\end{aligned}
$$

The detailed proof is omitted; it just consists in writing subtracting formula 56 for $K$ from the one for $K+e_{j}$ and reorganizing the terms.

We use now the CFL condition (45) and the definition of the coefficient $a_{K, L}^{n}$ to get, in particular, that

$$
\left(1-\sum_{-d \leq i \leq d} a_{K, K+e_{i}}^{n}\right) \geq 0, \forall K \in \mathcal{T}, \forall n<N
$$

so that we can take the absolute values in the formula given by Lemma 7.10 to get

$$
\begin{aligned}
\left|u_{K}^{n+1}-u_{K+e_{j}}^{n+1}\right| \leq \mid u_{K}^{n}- & u_{K+e_{j}}^{n}\left|\left(1-\sum_{-d \leq i \leq d} a_{K, K+e_{i}}^{n}\right)+\sum_{-d \leq i \leq d} a_{K, K+e_{i}}^{n}\right| u_{K+e_{i}}^{n}-u_{K+e_{i}+e_{j}}^{n} \mid \\
& +\sum_{-d \leq i \leq d}\left|a_{K, K+e_{i}}^{n}-a_{K+e_{j}, K+e_{j}+e_{i}}^{n}\right|\left|u_{K+e_{j}+e_{i}}^{n}-u_{K+e_{j}}^{n}\right|+\left|R_{K}^{n}\right|+\left|R_{K+e_{j}}^{n}\right|
\end{aligned}
$$

The last two terms are estimated by (57), whereas the first term of the second line is controlled by $(49)$ and (54). By summing this inequality over $K \in \mathcal{T}$ and $j \in\{-d, \ldots, d\}$ on the one hand and over $K \in \mathcal{T}^{\text {ext }}$, with $j=-d$ on the other hand (we use here Remark 7.3), we obtain

$$
\begin{aligned}
Q^{n+1} \leq & \sum_{K \in \mathcal{T}} \sum_{-d \leq j \leq d}\left(\left|u_{K}^{n}-u_{K+e_{j}}^{n}\right|\left(1-\sum_{-d \leq i \leq d} a_{K, K+e_{i}}^{n}\right)+\sum_{-d \leq i \leq d} a_{K, K+e_{i}}^{n}\left|u_{K+e_{i}}^{n}-u_{K+e_{i}+e_{j}}^{n}\right|\right) \\
& +\sum_{K \in \mathcal{T}_{\text {ext }}}\left(\left|u_{K}^{n}-u_{K+e_{-d}}^{n}\right|\left(1-\sum_{-d \leq i \leq d} a_{K, K+e_{i}}^{n}\right)+\sum_{-d \leq i \leq d} a_{K, K+e_{i}}^{n}\left|u_{K+e_{i}}^{n}-u_{K+e_{i}+e_{-d}}^{n}\right|\right) \\
& +C \Delta x\left(Q^{n}+B^{n}\right)+\sum_{K \in \mathcal{T}^{*}} a_{K, K+e_{d}}^{n}\left|u_{K+e_{d}}^{n}-u_{K}^{n}\right| .
\end{aligned}
$$

We have now to make a change of index $K+e_{i} \rightarrow K$ in the terms of the form $a_{K, K+e_{i}}^{n}\left|u_{K+e_{i}}^{n}-u_{K+e_{i}+e_{j}}^{n}\right|$ in the first two lines. It is needed of course to take care of the boundary terms. By straightforward algebraic manipulations we get the following lemmas. 
Lemma 7.11 (Change of indices on the whole mesh).

$$
\begin{aligned}
\sum_{K \in \mathcal{T}} \sum_{-d \leq j \leq d} \sum_{-d \leq i \leq d} a_{K, K+e_{i}}^{n}\left|u_{K+e_{i}}^{n}-u_{K+e_{i}+e_{j}}^{n}\right| \leq & \sum_{K \in \mathcal{T}} \sum_{-d \leq j \leq d-d \leq i \leq d} \sum_{K-e_{i}, K}\left|u_{K}^{n}-u_{K+e_{j}}^{n}\right| \\
& +\sum_{K \in \mathcal{T}_{\text {ext }}^{n}} a_{K, K-e_{d}}^{n}\left|u_{K}^{n}-u_{K-e_{d}}^{n}\right|-\sum_{K \in \mathcal{T}_{\text {ext }}} a_{K-e_{d}, K}^{n}\left|u_{K+e_{d}}^{n}-u_{K}^{n}\right| \\
& +C B^{n} .
\end{aligned}
$$

Lemma 7.12 (Change of indices for boundary terms).

$$
\begin{aligned}
\sum_{K \in \mathcal{T}^{\mathrm{ext}}} \sum_{-d \leq i \leq d} a_{K, K+e_{i}}^{n}\left|u_{K+e_{i}}^{n}-u_{K+e_{i}+e_{-d}}^{n}\right|= & \sum_{K \in \mathcal{T} \text { ext }} \sum_{d \leq i \leq d} a_{K-e_{i}, K}^{n}\left|u_{K}^{n}-u_{K+e_{-d}}^{n}\right| \\
& +\sum_{K \in \mathcal{T}_{\mathrm{ext}}} a_{K, K+e_{d}}^{n}\left|u_{K+e_{d}}^{n}-u_{K}^{n}\right|-\sum_{K \in \mathcal{T}_{\mathrm{ext}}} a_{K+e_{d}, K}^{n}\left|u_{K-e_{d}}^{n}-u_{K}^{n}\right| \\
& -\sum_{K \in \mathcal{T}_{\mathrm{ext}}} a_{K-e_{d}, K}^{n}\left|u_{K}^{n}-u_{K-e_{d}}^{n}\right| .
\end{aligned}
$$

Using the previous two lemmas with $(58)$ and using the property $(55)$, we finally obtain

$$
\begin{aligned}
Q^{n+1} \leq & (1+C \Delta t) Q^{n}+C(1+\Delta t) B^{n} \\
& +\sum_{K \in \mathcal{T}^{*}} a_{K, K+e_{d}}^{n}\left|u_{K+e_{d}}^{n}-u_{K}^{n}\right|+\sum_{K \in \mathcal{T}^{\mathrm{ext}}} a_{K, K-e_{d}}^{n}\left|u_{K}^{n}-u_{K-e_{d}}^{n}\right|-\sum_{K \in \mathcal{T}^{\mathrm{ext}}} a_{K-e_{d}, K}^{n}\left|u_{K+e_{d}}^{n}-u_{K}^{n}\right| \\
& +\sum_{K \in \mathcal{T}^{\mathrm{ext}}} a_{K, K+e_{d}}^{n}\left|u_{K+e_{d}}^{n}-u_{K}^{n}\right|-\sum_{K \in \mathcal{T}_{\mathrm{ext}}} a_{K+e_{d}, K}^{n}\left|u_{K-e_{d}}^{n}-u_{K}^{n}\right|-\sum_{K \in \mathcal{T}^{\mathrm{ext}}} a_{K-e_{d}, K}^{n}\left|u_{K}^{n}-u_{K-e_{d}}^{n}\right|
\end{aligned}
$$

With a simple change of indices, we observe that

$$
\sum_{K \in \mathcal{T}^{*}} a_{K, K+e_{d}}^{n}\left|u_{K+e_{d}}^{n}-u_{K}^{n}\right|=\sum_{K \in \mathcal{T}_{\text {ext }}} a_{K-e_{d}, K}^{n}\left|u_{K}^{n}-u_{K-e_{d}}^{n}\right|,
$$

so that we can gather all the terms as follows

$$
\begin{aligned}
Q^{n+1} \leq & (1+C \Delta t) Q^{n}+C(1+\Delta t) B^{n} \\
& +\sum_{K \in \mathcal{T}_{\text {ext }}}\left(a_{K, K-e_{d}}^{n}-a_{K+e_{d}, K}^{n}\right)\left|u_{K}^{n}-u_{K-e_{d}}^{n}\right|+\sum_{K \in \mathcal{T}_{\text {ext }}}\left(a_{K, K+e_{d}}^{n}-a_{K-e_{d}, K}^{n}\right)\left|u_{K+e_{d}}^{n}-u_{K}^{n}\right|,
\end{aligned}
$$

and using once again (54) and (49), we obtain

$$
Q^{n+1} \leq(1+C \Delta t) Q^{n}+C B^{n}+C^{\prime} \Delta t\left(Q^{n}+B^{n}\right)
$$

which implies the claimed estimate by multiplying the inequality by $\Delta x^{d-1}$. The proposition is proved, and so is the BV regularity theorem in the half-space case.

\subsection{Proof of Theorem 2.5}

Let us denote by $\Omega^{0}=\mathbb{R}^{d-1} \times(0,+\infty)$ the reference half-space we introduced in the previous section and by $\Gamma^{0}=\mathbb{R}^{d-1} \times\{0\}$ its boundary.

- The first step of the proof consists in considering the case where $\Omega$ has the following form

$$
\Omega=\left\{x \in \mathbb{R}^{d}, \quad x_{d}>S\left(x_{1}, \ldots, x_{d-1}\right)\right\},
$$

where $S: \mathbb{R}^{d-1} \rightarrow \mathbb{R}$ is a $\mathcal{C}^{3}$ and compactly supported parametrization of the boundary $\Gamma$.

We can then build a $\mathcal{C}^{2}$ diffeomorphism $\Psi: \mathbb{R}^{d} \rightarrow \mathbb{R}^{d}$ that maps $\Omega^{0}$ onto $\Omega$ and such that, for some $\xi_{\Omega}>0$ we have

$$
\Phi\left(\bar{x}, x_{d}\right)=(\bar{x}, S(\bar{x}))-x_{d} n(\bar{x}, S(\bar{x})), \quad \forall \bar{x} \in \mathbb{R}^{d-1}, \forall x_{d} \in\left(0, \xi_{\Omega}\right) .
$$


where $n$ is the outward unit vector field of the domain $\Omega$. With this change of variable we can set

$$
w(t, x):=u(t, \Psi(x)), \forall t \in(0, T), \forall x \in \Omega^{0},
$$

and prove that $w$ solves a transport problem in $\Omega^{0}$ with initial data $w^{0}(x)=u^{0}(\Psi(x))$, boundary data $w_{b}(t, x)=u_{b}(t, \Psi(x))$ and the advection field $\mathbf{v}^{0}$ defined by

$$
\mathbf{v}^{0}(t, x)=[(\nabla \Psi)(x)]^{-1} \mathbf{v}(t, \Psi(x)), \quad \forall t \in(0, T), \forall x \in \Omega^{0} .
$$

The regularity of $\Psi$ shows that $\mathbf{v}^{0}$ inherits the regularity properties of $\mathbf{v}$ (it is however not divergence free in general), and moreover the change of variable we used maps the inflow part of the boundary for $\mathbf{v}^{0}$ onto the inflow part of the boundary for $\mathbf{v}$. Theorem 7.8 then gives an estimate of $w$ in $L^{\infty}\left(0, T, \mathrm{BV}\left(\Omega^{0}\right)\right)$ depending on the total variations of $w^{0}$ and $w_{b}$. Coming back to $u$ by the change of variable gives the corresponding estimate on $u$.

- Let us now prove the claim on a general bounded $\mathcal{C}^{3}$ domain. We first introduce a finite covering of $\Omega=\cup_{1 \leq i \leq I} \Omega \cap B\left(x_{i}, r\right)$ in such a way that for any $i \in\{1, \ldots, I\}$ the set $\Omega \cap B\left(x_{i}, 2 r\right)$ is the intersection of $\Omega$ with an half-space of the form (59), up to some rotation. We also choose a $\tau>0$ such that $\tau\|\mathbf{v}\|_{L^{\infty}\left(\Omega^{T}\right)} \leq r$.

Since Problem (1) is linear, we can assume that $u^{0}$ (resp. $u_{b}$ ) is supported in $\Omega \cap B\left(x_{i}, r\right.$ ) (resp. in $\left.\Gamma^{T} \cap\left((0, T) \times B\left(x_{i}, r\right)\right)\right)$. A standard estimate shows that, thanks to the choice of $\tau$, the corresponding solution is supported in $\Omega \cap B\left(x_{i}, 2 r\right)$ during the time interval $(0, \tau)$. Hence, we can apply the claim proved in the first step and deduce that the solution is BV during the time interval $(0, \tau)$ with the suitable estimate. Using a partition of unity we can then conclude that, for any data $u^{0}$ and $u_{b}$, we have

$$
\sup _{t \in[0, \tau]} \operatorname{TV}_{\Omega}(u(t, .)) \leq C\left(\left\|u^{0}\right\|_{\mathrm{BV}(\Omega)}+\left\|u_{b}\right\|_{\mathrm{BV}\left(\Gamma^{T}\right)}\right) .
$$

Applying the same result on the time interval $[\tau, 2 \tau]$, since $\tau$ only depends on $\Omega$ and $\mathbf{v}$ we get

$$
\sup _{t \in[\tau, 2 \tau]} \operatorname{TV}_{\Omega}(u(t, .)) \leq C\left(\|u(\tau, .)\|_{\mathrm{BV}(\Omega)}+\left\|u_{b}\right\|_{\mathrm{BV}\left(\Gamma^{T}\right)}\right)
$$

and combining the two estimates finally gives

$$
\sup _{t \in[0,2 \tau]} \operatorname{TV}_{\Omega}(u(t, .)) \leq C^{2}\left(\left\|u^{0}\right\|_{\mathrm{BV}(\Omega)}+\left\|u_{b}\right\|_{\mathrm{BV}\left(\Gamma^{T}\right)}\right) .
$$

By a simple induction, we prove that

$$
\sup _{t \in[0, T]} \operatorname{TV}_{\Omega}(u(t, .)) \leq C^{T / \tau}\left(\left\|u^{0}\right\|_{\mathrm{BV}(\Omega)}+\left\|u_{b}\right\|_{\mathrm{BV}\left(\Gamma^{T}\right)}\right)
$$

Finally, we known that if $u^{0}$ and $u_{b}$ are both equal to a given constant then the solution $u$ is itself constant. Hence, by standard arguments the right-hand side in the last inequality can be replaced by $C\left(\left\|\gamma_{\Gamma} u^{0}-u_{b}(0, .)\right\|_{L^{1}}+\mathrm{TV}_{\Omega}\left(u^{0}\right)+\mathrm{TV}_{\Gamma^{T}}\left(u_{b}\right)\right)$ for some other value of $C$.

\section{Acknowledgments}

This work has been carried out in the framework of Archimède Labex (ANR-11-LABX-0033) and of the A*MIDEX project (ANR-11-IDEX-0001-02), funded by the "Investissements d'Avenir" French Government programme managed by the French National Research Agency (ANR).

\section{References}

[AFP00] L. Ambrosio, N. Fusco, and D. Pallara. Functions of bounded variation and free discontinuity problems. Oxford Mathematical Monographs. The Clarendon Press, Oxford University Press, New York, 2000. 
[Amb00] L. Ambrosio. Geometric evolution problems, distance function and viscosity solutions. In Calculus of variations and partial differential equations (Pisa, 1996), pages 5-93. Springer, Berlin, 2000.

[BF13] F. Boyer and P. Fabrie. Mathematical Tools for the Study of the Incompressible Navier-Stokes Equations and Related Models. Springer New York, 2013.

[BGP05] D. Bouche, J.-M. Ghidaglia, and F. Pascal. Error estimate and the geometric corrector for the upwind finite volume method applied to the linear advection equation. SIAM J. Numer. Anal., 43(2):578-603 (electronic), 2005 .

[BlRn79] C. Bardos, A. Y. le Roux, and J.-C. Nédélec. First order quasilinear equations with boundary conditions. Comm. Partial Differential Equations, 4(9):1017-1034, 1979.

[Boy05] F. Boyer. Trace theorems and spatial continuity properties for the solutions of the transport equation. Differential Integral Equations, 18(8):891-934, 2005.

[Boy12] F. Boyer. Analysis of the upwind finite volume method for general initial- and boundary-value transport problems. IMA Journal of Numerical Analysis, 32(4):1404-1439, dec 2012.

[CH99] C. Chainais-Hillairet. Finite volume schemes for a nonlinear hyperbolic equation. Convergence towards the entropy solution and error estimate. M2AN Math. Model. Numer. Anal., 33(1):129-156, 1999.

[CR15] R. Colombo and E. Rossi. Rigorous estimates on balance laws in bounded domains. Acta Mathematica Scientia, 35(4):906-944, jul 2015

[CVV00] Y. Coudière, J.-P. Vila, and P. Villedieu. Convergence d'un schéma volumes finis explicite en temps pour les systèmes hyperboliques linéaires symétriques en domaines bornés. C. R. Acad. Sci. Paris Sér. I Math. 331(1):95-100, 2000.

[DE06] J. Droniou and R. Eymard. A mixed finite volume scheme for anisotropic diffusion problems on any grid. Numer. Math., 105(1):35-71, 2006.

[Des04a] B. Després. An explicit a priori estimate for a finite volume approximation of linear advection on nonCartesian grids. SIAM J. Numer. Anal., 42(2):484-504 (electronic), 2004.

[Des04b] B. Després. Lax theorem and finite volume schemes. Math. Comp., 73(247):1203-1234, 2004.

[DL89] R. J. DiPerna and P.-L. Lions. Ordinary differential equations, transport theory and sobolev spaces. Inventiones Mathematicae, 98(3):511-547, oct 1989 .

[DL11] F. Delarue and F. Lagoutière. Probabilistic analysis of the upwind scheme for transport equations. Arch. Ration. Mech. Anal., 199(1):229-268, 2011.

[DLV16] F. Delarue, F. Lagoutière, and N. Vauchelet. Convergence order of upwind type schemes for transport equations with discontinuous coefficients. Preprint, arXiv:1602.05746, 2016.

[Dro03] J. Droniou. Error estimates for the convergence of a finite volume discretization of convection-diffusion equations. Journal of Numerical Mathematics, 11(1):1-32, jan 2003.

[Kuz76] N. N. Kuznetsov. The accuracy of certain approximate methods for the computation of weak solutions of a first order quasilinear equation. Ž. Vyčisl. Mat. i Mat. Fiz., 16(6):1489-1502, 1627, 1976.

[Mer08] B. Merlet. $L^{\infty}$ - and $L^{2}$-error estimates for a finite volume approximation of linear advection. SIAM $J$. Numer. Anal., 46(1):124-150, 2008.

[MV07] B. Merlet and J. Vovelle. Error estimate for finite volume scheme. Numer. Math., 106(1):129-155, 2007.

[OV06] M. Ohlberger and J. Vovelle. Error estimate for the approximation of nonlinear conservation laws on bounded domains by the finite volume method. Math. Comp., 75(253):113-150 (electronic), 2006.

[Pet91] T. E. Peterson. A note on the convergence of the discontinuous Galerkin method for a scalar hyperbolic equation. SIAM J. Numer. Anal., 28(1):133-140, 1991.

[SS16] A. Schlichting and C. Seis. Convergence rates for upwind schemes with rough coefficients. Preprint, arXiv:1606.09156, 2016.

[Vig96] M.-H. Vignal. Convergence of finite volumes schemes for an elliptic hyperbolic system with boundary conditions. In Hermes F. Benkhaldoun et R. Vilsmeier, editor, proceeding of the First International Symposium on Finite Volumes for Complex Applications, pages 145-152. Hermès Paris, 1996.

[VV03] J.-P. Vila and P. Villedieu. Convergence of an explicit finite volume scheme for first order symmetric systems. Numer. Math., 94(3):573-602, 2003. 